\title{
Pulse width modulation: the language of the brain
}

\section{Robert Alan Brown}

Mattapoisett, Massachusetts, USA

\section{Abstract}

Music, speech, dance, echo location, sonar, telegraphy, radio, and the original Fax telegraphics and television are all based upon timing and rhythm. Pulse Width Modulation (PWM) is the science behind rhythm because it can define rhythm and the information within rhythm, precisely. So it stands to reason that the brain uses PWM to produce and understand music and speech and deal with these the other movement and messaging processes involving rhythm. PWM is a versatile electrical process used in control systems from light dimmers to spectrometers. PWM is based upon the measurement and/or the control of the time of events (spikes) within repeating periods in a carrier frequency of timed pulses. In the case of light dimmers, the PWM controller turns the light on at the beginning of each repeating (periodic) cycle in the $60 \mathrm{~Hz}$, alternating current (AC) carrier frequency, and turns it off early in each cycle to make the light appear dim, and turns off the light near or at the end of each cycle to make the light appear bright. In very simple muscles, like those found in the first animals like the crinoids, the degree of extension and flexion can be determined by the time between when these movements are started (turned on) and when they are stopped (turned off) within the repeating cycles of a PWM carrier frequency. A very simple muscle with only two muscle cells can be used as a high frequency spike generator. And a set of these muscle circuits can be used as a frequency divider that produces lower frequency global periodic carrier pulse train frequency needed in PWM. Timed pulses within these global periodic carrier pulses can create an output pulse train that also produces a restoring pulse for each output pulse resulting in a symmetrical, zero-sum computation that keeps order and balance in the PWM controller. The time information within these periodic carrier pulse cycles allow multiple muscles to work simultaneously with slightly different starting and finishing times to produce the constant acceleration and deceleration seen in eye saccades and almost all other animal movements produced by the brain. Bi-directional frequency dividers can be used as up/down counters that store information as short term and long-term latent memories of pulse times that can be retrieved later by the carrier pulse train to create and transmit messages within the brain, without movement, that determine the overall behavior of an animal.

\section{Keywords}

Pulse width modulation, brain waves, rhythm, information, order, symmetry, muscle, ternary computation, frequency generator, frequency divider, eye saccades, spike timing, spike counter, telegraphy, timer, muscle antagonism, motion sequence, language, bi-directional frequency divider, temporary memory, permanent memory, vision sensor

\section{Author Contact}

Robert Alan Brown, Mattapoisett, MA. 02739, USA E-mail: spikeperson@comcast.net

\section{Author Summary}

Music, speech, and dance use rhythm to convey information. In order to have rhythm, there has to be a periodic carrier beat. But a carrier beat contains no information. The carrier frequency is just a timing framework that can be used as a scanner that can collect information. In order to have information in the beat there has to be something that changes (modulates) within the beat, forming a system that operates using pulse width modulation (PWM). So in PWM, the beat is 
Pulse width modulation

the carrier of information, and the information is the timing of events within the beat. In PWM, information is introduced by a movement, tone, or other change in state that occurs (or does not occur) within the periods of the beat. So I show how the simplest two-cell muscle can produce the searching (scanning) motion needed to find food. These muscle units need to be arranged in an antagonistic (symmetrical) arrangement resulting in a ternary (three-value number system) of $+1,0,-1$, which forms a symmetrical, zero-sum pulse width modulation carrier frequency that can be reset (homed) periodically to restore the zero sum. This fault detection and correction process creates order, and reduces unusable information in the system. The neural circuit needed to drive the muscle can be used as a spike generator that can be used to synchronize the movement of multiple muscles. Multiple two-cell muscle circuits can be used together as a frequency divider that can be used to generate the low frequency pulse timing framework (carrier frequency) needed for PWM. More muscle cells can be added to the two cell muscle forming a multi-cell muscle that is capable of producing greater motion with higher resolution when driven by the spike generator and frequency divider. Multiple muscles can be coordinated by the low frequency timing pulse framework (carrier) in PWM to produce useful motion sequences that sense and avoid obstacles and produce the constant acceleration and deceleration measured in human eye saccades. Extended range frequency dividers can be made bi-directional, and be tapped so they can store and retrieve permanent information, and transmit the messages to other parts of the brain needed to control the behavior of an animal using PWM.

\section{Introduction}

The animal kingdom came into being over 500 million years ago with the appearance of muscles in organisms like the crinoids that created motion to capture food. These basal animals looked like plants with five branches. But rather than just waiting for food to float onto its branches, these animals moved their branches up and down. This created an outward flow of water that brought up potential food from on or near the bottom, and brought down food floating on or near the surface of the water. This movement greatly increased the opportunity of the animal to obtain nourishment, and has allowed descendants of these animals to survive to the present day. I show in this paper how this muscle movement can be created and controlled using PWM. In a previous paper (Brown R. A., 2016), I show how another ancient animal that exists today, called a Limpet, can use PWM to produce movement and navigate in a grazing motion that allows it to return to a home position groove it has ground into a rock in shallow water. In the present paper I show in greater detail how the muscles work in an animal like the crinoid, and how muscles can evolve to produce the smooth movements of the eye called saccades.

Systems using PWM represent information as time extents. So they could be called "time machines". The earliest time machine was the Antikythera found in recent times in a shipwreck that occurred over two thousand years ago in the Aegean Sea. It was very much like modern clocks or wrist watches that show the phases of the Moon, seasons, and other celestial events using planetary gearing. But instead of a precise timing mechanism like a balance staff, it was operated by a hand crank that allowed it to go backward and forward in time. This allowed its builders to adjust its planetary gearing (differentials) to match the celestial events recorded previously. So when it was advanced forward from the then present time it could be used to forecast future celestial events. The next important time machine was the tide predictor created by Thomas Watson (Lord Kelvin) and others, without the knowledge of the Antikythera, using ropes and pulleys in place of differentials. It was also constructed to match past records, and was advanced manually to predict future tide levels. 
Pulse width modulation

Another important time machine was the Differential Analyzer developed by Vannevar Bush at MIT in the 1930's. It used differentials to sum the output of multiple ball integrators to calculate the trajectories of projectiles, for example. It also used a global timed drive to integrate the output from the multiple ball integrators. This architecture became the basis of the Mark X naval gun controllers used in the Second World War. These highly successful mechanical analogue computers were superseded by digital computers over fifty years ago.

I developed and was granted a US patent on a mechanical analogue computer a little over fifty years ago called a Demand Register. It was used to compute the logarithmic average power used by a utility customer, and was somewhat based upon the architecture of the Differential Analyzer. However I used a periodic timing mechanism to measure the output of the register instead of a ball integrator, and subtracted a fixed portion of the output from the input from the watthour meter to produce the register output in periodic steps (Brown R. A., 1967). This periodic measurement and subtraction process is the basis of Pulse Width Modulation.

Morse code telegraphy originated over 150 years ago using PWM as its foundation. A previous paper, (Brown R. A., 2011) describes a generalized PWM spike timing process that transmits messages from one region to another over just a few conductors using pulse width information, much like a telegraph system. This process meets the demands of the central nervous system where many sensors must communicate with many brain neurons through the highly restrictive paths through the spinal cord for example. Also, PWM was used in the earliest Fax telegraphics machines to transmit "Wanted" images of cattle rustlers and horse thieves in the old west. So PWM was used exclusively for transmitting and receiving remote text and image information since before the Civil War in America until it was replaced with Pulse Code Modulation (PCM) with the widespread use of computers after the Second World War. Information is transmitted using PCM as a series of binary numbers instead of the time extent codes previously used in PWM telegraphy. Other wellknown information systems that are still used to transmit information are Amplitude Modulation (AM), where information is transmitted by variations in the amplitude of as audio signal, and Frequency Modulation (FM), where information is transmitted by variations in a frequency representing an audio signal.

In a paper (Bahill, Clark, \& Stark, 1975), the movements of the human eye (saccades) were measured over a wide range of eyeball rotations. The best fit for the data was made using an increasing rotational velocity (constant acceleration) of the eyeball to the midpoint of the motion, and decreasing rotational velocity (constant deceleration) to the end of the motion. The midpoint of the saccade was determined with the help of a pulse width modulator controller (Bahill, Clark, \& Stark, 1974). The pulse-width modulator was part of an analogue optical tracking system typically used in guided missiles. The question is: how can this optical tracking be carried out using entirely biological elements? This paper attempts to answer these questions by showing, progressively, the evolution of muscle circuits that produce useful motion using PWM that can be used to produce the constant acceleration/deceleration eye movements and other movements made by animals.

So, I started with the simplest muscle that could have been used in the first animals that produced a motion having a constant velocity like a clock or timer. But muscles must act together in antagonistic pairs. This requires a symmetrical process in which a muscle cell connected to a given joint extends whenever another muscle cell connected to that joint contracts. The first rule of PWM is that every pulse onset (start) spike must have an offset (stop) spike forming a positive 
Pulse width modulation

pulse of some duration. In the symmetrical, zero-sum PWM used in this paper, every positive onset spike has an opposite (negative) onset and offset spike, forming a negative pulse. And every positive starting pulse train will have a negative starting pulse train. This leads to a ternary (three value) number system consisting of $1,0,-1$ rather than the binary (two value) number system consisting of 1, 0 used in digital computers today. This allows the sum of values in this symmetrical ternary system to stay at or close to zero, which works perfectly with antagonistic muscle pairs.

When a constant velocity is employed in a motion actuator (muscle), the pulse time corresponds directly to the distance moved in that pulse time. I show the design of the pulse width modulation controller that produces constant velocity motion pulses. I show a set of evolving systems that use this PWM controller to produce useful behavior in a simple proto animal model like a crinoid that can avoid and remember objects in its environment. If an animal muscle moves with an increasing velocity to the midpoint of its motion and a decreasing velocity to the end of its motion that is the mirror image of the increasing velocity, as in the Bahill paper, the distance the muscle moves is directly related to the square of the timed pulse, also. I show how this constant acceleration/deceleration can be created by multiple constant velocity muscles that are started and stopped at different times, and have their outputs added together using PWM. This PWM process is particularly well suited for the control and coordination of animal muscles, and robot actuators as well.

I propose the view that the foundational element of information in the brain is the pulse time and position within a period within a carrier, and that the set of these pulses within this carrier pulse train contains all of the information processed in the brain. The transmission of these information pulses throughout the nervous system is carried out as spike trains that operate within the PWM carrier time framework. These spike trains produce pulse trains that determine the movement of the animal models. These carrier pulse trains also act as a scanner, which form the raster of an object sensing system that connects the animal to its environment, cyberneticly. The key to this process is the use of timers as the working, temporary, and long-term memory devices in the system. A more detailed understanding of the use of Pulse Width Modulation in the brain is provided in a previous paper (Brown R. A., 2016).

\section{Methods and Materials}

The method used in this paper to decipher the operation of the brain is to start with an understanding how a muscle could work in the first and simplest animals, the crinoids, for example. Then I figured out how these circuits could evolve to carry out more complex and useful functions such as avoiding objects, forming memories, and creating smooth motions. In every step of the way I drew models with logic circuits that used the previous circuits and included some simple change to produce the new and useful results, which is the likely process used in biological evolution.

Almost every circuit is a complete machine model. I show how these models respond to different input (sensor) states by showing a few examples of different input states for each machine. I have put these models into action using animations that showed the actual logic states and the resulting output states. This acted as a rigorous check of the logic circuits, and provides an excellent tutorial on their function. Unfortunately, these animations cannot be included in this paper. But they are available upon request.

In this paper, the normally open contacts of the relays represent excitatory neurons, and the normally closed contacts represent inhibitory neurons. The muscles are in fact timers that act as integrators that express a physical movement. Since the muscles are connected in series, I show their movements being summed physically by the symbol 
Pulse width modulation

for a differential in a manner similar to that used by Vannevar Bush's Differential Analyzer.

The models shown this paper are drawn with the spike sources such as sensors (encoders) and the system timing bus on the left side of the page, the muscles and memories (decoders) in the middle of the page, and the pulse trains (output of the decoders) on the right side of the page in the manner of the Bush Differential Analyzer. The spikes produced on the left hand side of the page must be equal to the onset and offset spikes in the pulses on the right hand side of the page, forming a balanced set of equations. These pulse trains define the rhythmical behavior expressed by the model. Graphical analysis of the pulse trains produces the resultant trajectories (movements) of the models that are based upon the kinematics of the animal model. All of the pulse trains and motion trajectories in the paper are based upon rigorous calculations based upon spike times, and their accuracy can be checked using the numerical date in the computer generated graphics.

The models shown in this paper can be used to create computers programs that use PWM to create motion sequences and messages. Computers have a built-in high frequency generator (clock) that operates at a very high frequency, and have high resolution up/down counters that can simulate muscles and memories. Starting with logic circuits like those shown in this paper is a straight forward way to write computer programs that perform PWM.

\section{Results}

This paper shows how the brain could have evolved from the neural circuits needed to drive the muscles of the first animals, since these simple Pulse Width Modulation circuits are shown to provide the memory, timing and symmetry needed for complex motion and messaging. I have shown the exact time extent (duration) circuits that produce spike trains and decode them into useful actions. The memory and plasticity of the control system is changed according to the spike time durations created by sensing the location of the objects it encounters and stored within sets of memory registers made of the circuits used to drive muscles. These memories are created and stored while the connections in these circuits remain unchanged. This contrasts to the prevalent view in neuroscience that memory and plasticity is the result of changes at a single synaptic connection, and offers a fundamental change in the way of thinking about how the brain works.

A general or even universal law of information processing is that if complexity (information) is created (encoded) within the framework of a regular (constant) pattern, the original information can be decoded (restored) by reapplying the original regular pattern. This is seen clearly in television, where the information in a scene is detected within the regularly repeating raster pattern of a camera. Then the scene can be reproduced at a receiver when it is exposed to this information, sequentially, and if the receiver produces the original scan pattern of the camera. Another example of this principle is shown in how voice and music are broadcast over the radio. The varying audio signal from a microphone is added to a much higher and constant carrier frequency, which is added to a much higher and constant broadcast frequency that can be transmitted over great distances. A radio receiver tuned to this broadcast frequency produces and subtracts the broadcast frequency and the original carrier frequency, which leaves just the original varying audio signal of voice and music. This signal is amplified and made audible by the speaker in the radio receiver.

Another example of the use of a carrier frequency to transmit information is seen the Automatic Send and Receive (ASR) Teletype Machine in my office years ago. It sent and received data over the phone lines to a time share computer in another city. I could hear the steady click, click, click,..., of its carrier frequency at five Hz that was synchronized with 
Pulse width modulation

another ASR at the remote computer as it stood by ready to send or receive information. Then I could hear the steady sound of its punched paper tape printer when it was actually sending or receiving a message using the ASCII character code.

The key feature of what is regular in these information processes is the constancy of the carrier. The TV raster is a constantly repeating pattern. It never changes. The carrier and broadcast frequencies in radio are ever constant. The High Frequency Spike Train and the Low Frequency Period Pulse Train in pulse width modulation remain a constant.

It may be that all complex systems need this constant base carrier. Our Federal Constitution and the laws of the land are supposed to be an enduring constant that determines the behavior of our fellow citizens. In computers, the same operating system controls many different applications, successfully. Our written and spoken language is mostly fixed and unchanging; making it possible for us to communicate information of unimaginable complexity with other people if they apply what is fixed and unchanging in our language to our message.

The passage of time is one of the most regular (consistent) processes in the non-relativistic universe. So, a sentient organism can communicate successfully with its environment by finding and encoding the information (complexity) in its environment using the periodic beat (regularity) of the carrier frequency in the timing framework within PWM, and then decoding this information sequentially using the carrier frequency within PWM. This allows an animal to act meaningfully upon this information in that environment.

The constancy of our vocabulary and grammar allows us to exchange information. We call this our language. The constancy of the timing carrier framework and protocols in pulse width modulation may allow an organism to exchange information with its environment. So pulse width modulation may be called the language of the brain. 


\section{Discussion}

This discussion shows how a two-cell muscle can evolve in to a spike generator and frequency divider that can be used with a multi-cell muscle to form a useful motion and messaging system.

\subsection{The antagonistic two-cell muscle unit}

Many, if not all, of the activities in a living animal, such as the beating heart, breathing, blinking, etc. require a steady, repeating signal. These signals also exist in the form of brain waves (Buzsaki, 2006). These signals can be created by time based pulse trains, which automatically return to their original state and repeat themselves. This section shows how spikes, timing units, and muscle can form a useful machine using timed pulses.

\subsubsection{A two-cell positive muscle unit}

The simplest muscle unit consists of two cells; a Positive Max Muscle Cell (1L+) and a Positive Zero Muscle Cell (1R+) with ligaments that are wrapped around a Rotor connected to an Output Arm, as shown in Figure 1.

\section{Positive Pulse Muscle Unit (Moving Toward Max +)}

Step 8. After arriving at Zero, a spike at the (T1+) Terminal from the Zero Terminal (Z-) causes the Max Latch (1L+) to close, and Zero Muscle (1R+) to open, causing the Rotor Contact Arm to start moving CCW (L) away from Zero (Z-) toward the Max Position $(\mathrm{X}+)$, as shown.

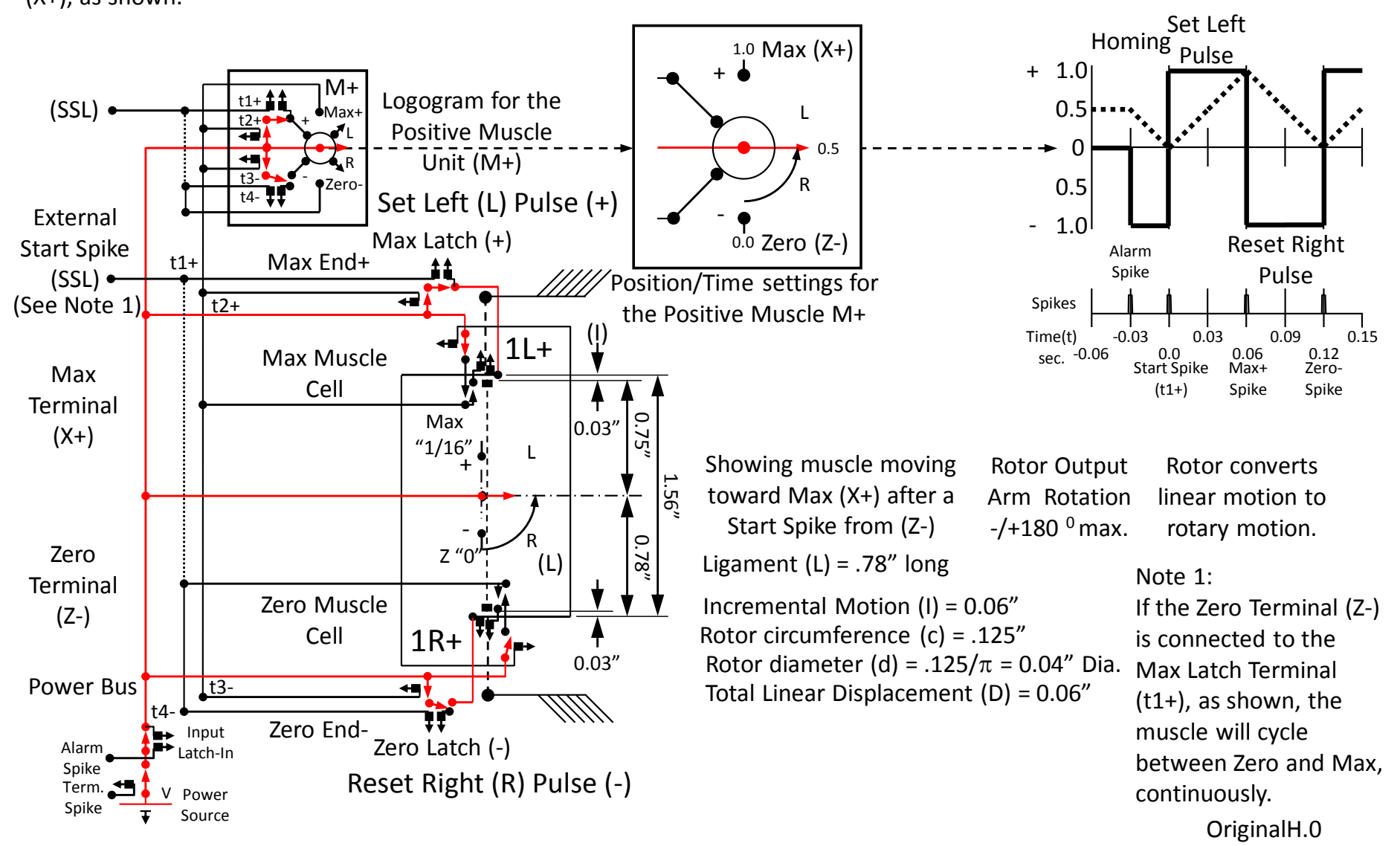

Figure 1 The muscle Output Arm starts to "Home" towards Zero (Z-) when the muscle is connected to a Power Source

(energized) by the closure of an Input Latch by an Alarm Spike, When it arrives at Home it starts to oscillate between the Zero

position and the Max position until there is a Termination Spike at the Input Latch connected to the Power Source.

The two-cell Positive Muscle (M+) starts to rotate its Output Arm clockwise home to its zero position when it is connected to the Power Source by an Alarm Spike. When the Output Arm gets to zero, a spike on the Zero Terminal (Z-) closes the Max Latch (+). Because the Zero Terminal (Z-) is connected to the Left Start Spike Terminal (SSL), the Output Arm to start rotating counter-clockwise toward it maximum position. When the Output Arm gets to its Maximum Position $(\mathrm{Max}+)$, the Max Terminal $(\mathrm{X}+)$ releases the Zero Latch (-) causing the Output arm to start rotating toward the Zero Position (Z-), again.

\subsubsection{Two-cell negative muscle unit}


A complete time axis in a system with PWM requires two semi-axes: A positive left-turning (counter clockwise) semiaxis shown above in Figure 1, and a negative right-turning (clockwise) semi-axis shown below in Figure 2.

\section{Negative Pulse Muscle Unit (Moving Toward Max -)}

Step 8. After arriving at Zero + , a spike at the (t1-) Terminal from the Zero Terminal $(\mathrm{Z}+)$ closes the Max Latch. The Max Muscle Cell (1R-) starts to close, causing the Rotor Output Arm to start to move CW (R) away from Zero (Z+) toward the Max Position (X-), as shown.

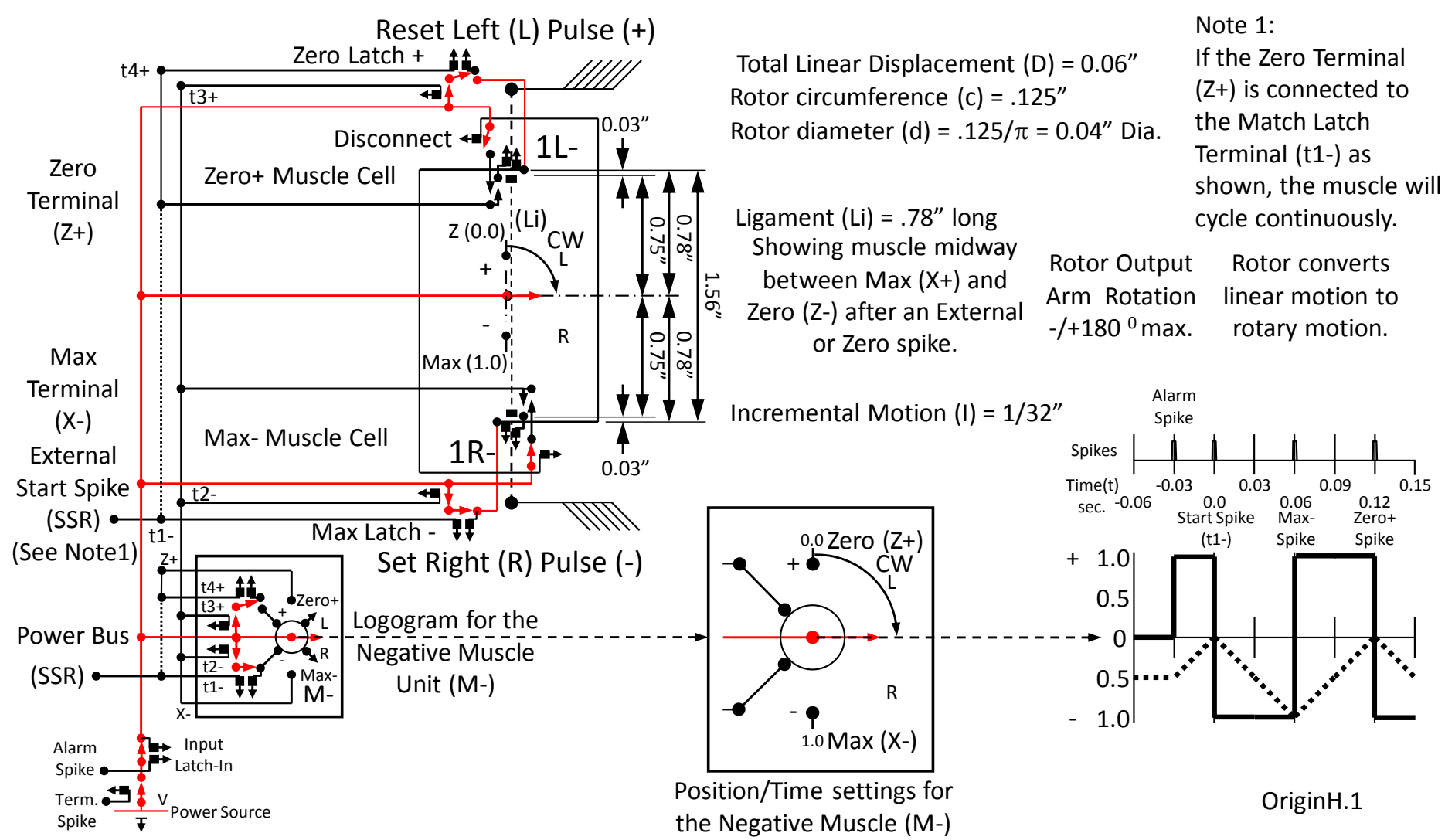

Figure 2 The two-cell Negative Muscle keeps repeating a motion in the opposite direction Of the Positive Muscle until stopped by a Termination Spike

The negative muscle operates through the same steps as the positive muscle. But it sets clockwise and resets counter-clockwise, which is in the opposite direction of the positive muscle.

\subsubsection{Computing with a symmetrical four-cell positive and negative muscle group}

The positive and negative two-cell muscle units can be put together to form a single symmetrical four cell differential muscle group with an output that is equal to the sum of the outputs of each, as shown in Figure 4. 
Four Cell Differential Muscle (Homed)

Step 1+. After Homing to Zero-, the Muscle Cell (1L+) on the Max + End is open and the Muscle Cell (1R+) on the Zero - End is latched closed, as shown.

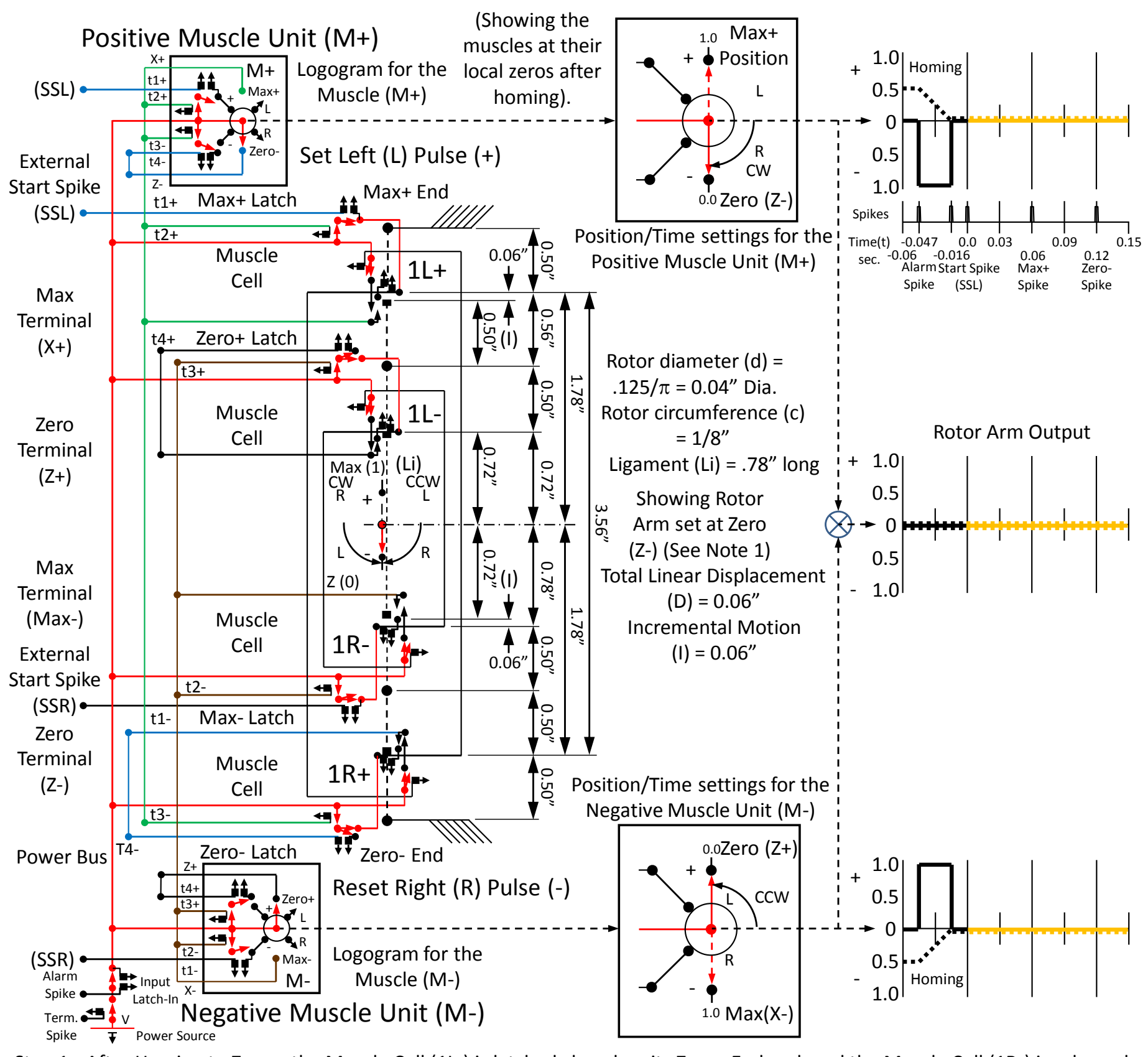

Step 1-. After Homing to Zero+, the Muscle Cell (1L-) is latched closed on its Zero+ End end, and the Muscle Cell (1R-) is released open on its Max- End, as shown.

AgonistA.1

Figure 3 The symmetrical, antagonistic muscle group is made up of a Positive Muscle Unit (M+) and a Negative Muscle Unit

(M-). So the sum of the Positive Muscle Unit (M+) and the Negative Muscle Unit (M-) is zero after both units are homed to zero according to the equation $0+0=0$, as shown.

The state of the Muscle Cells is shown for both Muscle Units being homed at zero. When the Muscle Units are connected into one muscle group they must be started by the External Start Spike (SSL) and/or (SSR).

\subsubsection{Both two-cell muscle units acting together}

If both muscle units are started at the same time by spikes on the Start Spike Terminals (SSL) and SSR), the Rotor Output Arm remains at zero, as shown below in Figure 4. 
Four Cell Differential Muscle (Arrived at Max)

Step $3+$. When the Muscle Cell $(1 \mathrm{~L}+)$ closes there is a spike at the $(\mathrm{t} 2+)$ Terminal from the Max Terminal $(\mathrm{X}+)$, which releases the Max Latch+, and releases Zero Latch +, which energizes the Muscle Cell $(1 \mathrm{R}+)$ and releases the Muscle Cell ( $1 \mathrm{~L}+)$, causing their Ligaments to start moving down.

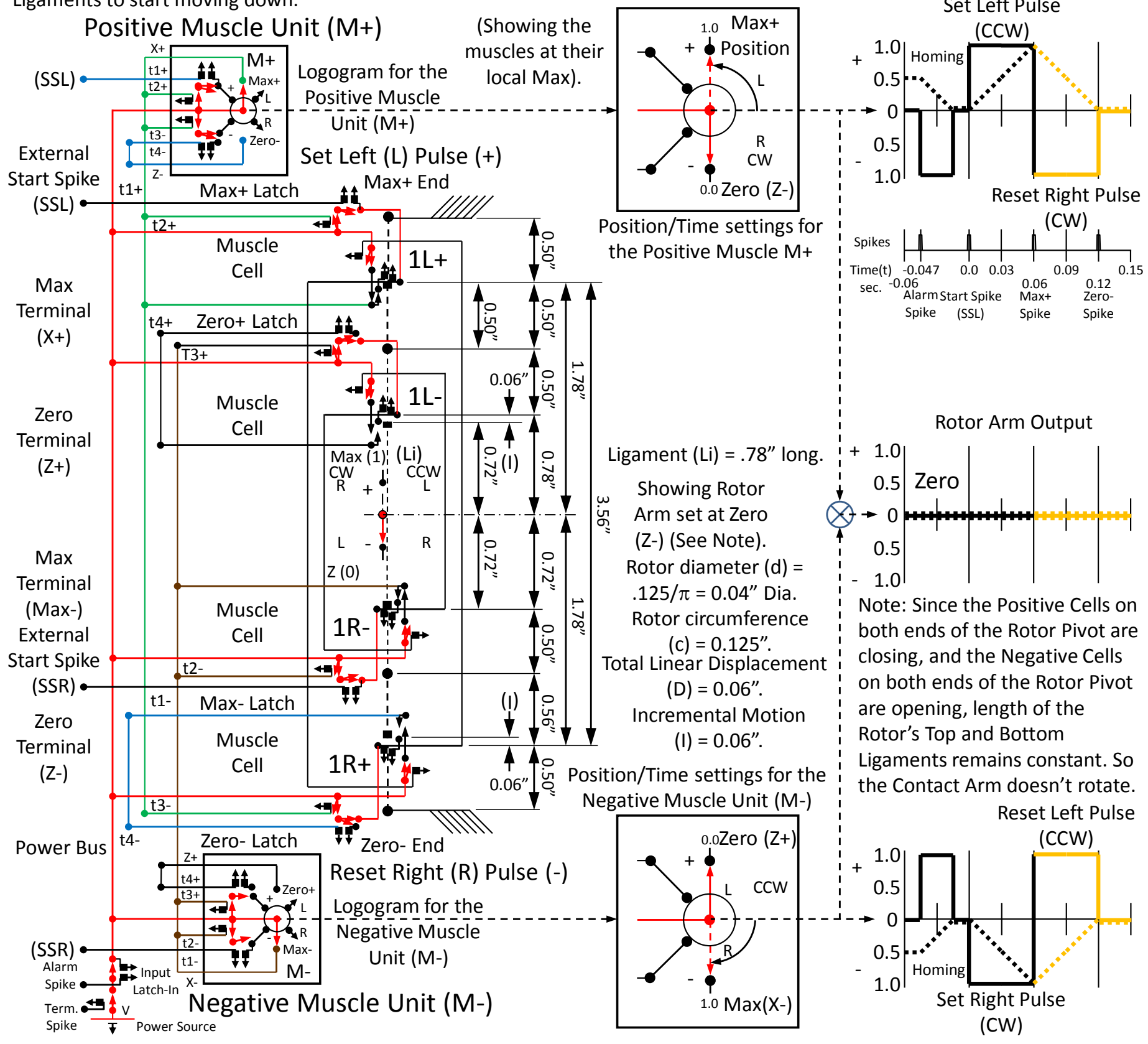

Step 3-. When the Muscle Cell (1R-) closes there is a spike at the (t2-) Terminal from the Max Terminal (X-), which releases the Max Latch -, and releases the Zero Latch -, which energizes the Muscle Cell (1L-) and releases the Muscle Cell (1R-), causing their Ligaments to start moving up.

AgonistB.1

Figure 4 When there is a spike at the Left Start Spike (SSL) and the Right Start Spike (SSR), simultaneously, both

of the Positive and Negative Muscle Units act together. And the output of the symmetrical muscle group is zero according to the equation $1+(-1)=0$.

When linear actuators such as muscles are connected in series, the output extent is equal to the sum of their individual output extents. This summing is represented by the symbol of the differential shown above. The state of the Muscle Cells is shown in their position for maximum output.

\subsubsection{The positive four-cell muscle unit acting alone}

If the Positive Muscle Unit is started by the Start Spike (SSL), and the Negative Muscle Unit is not started, the Symmetrical Muscle Group will produce a positive output, as shown in Figure 5. 
Step 3+. When the Muscle Contact ( $1 \mathrm{~L}+)$ on the Max+ End closes, the Max Latch (+) is released, cutting power to the Muscle (1L+) and the Zero Latch (-) is released sending power to the Muscle (1R+).

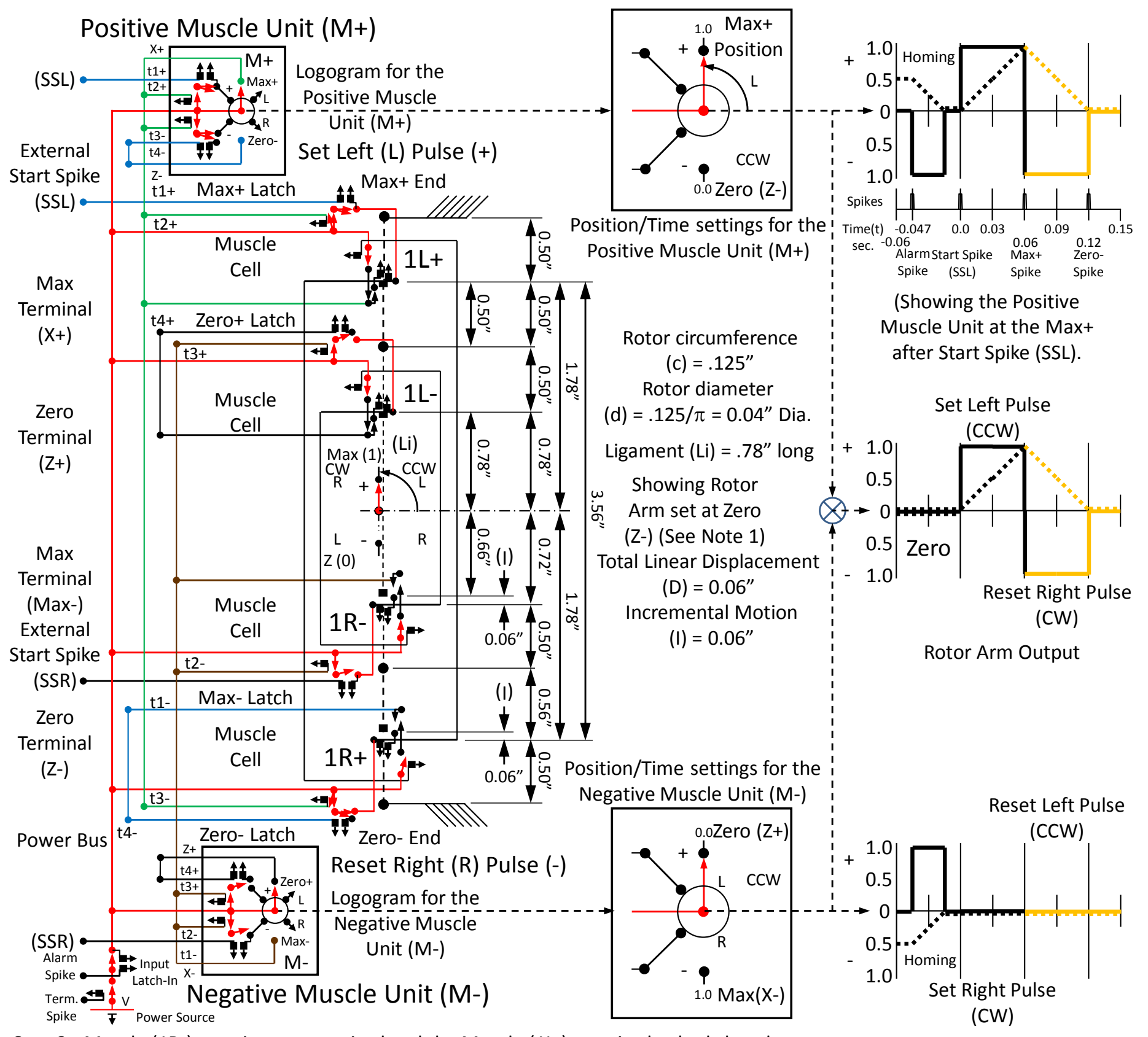

Step 3-. Muscle (1R-) remains un-energized and the Muscle (1L-) remains latched closed.

Figure 5 The symmetrical four-cell muscle group computes the sum of $1+(0)=1$.

The Output Arm rotates to the left because the Max Muscle Cell (1L+) of the Positive Unit $(\mathrm{M}+)$ closes, and its Zero Muscle Cell (1R+) opens. The extent of the Negative Muscle (M-) does not change. So it acts like extended ligaments of the Positive Muscle (M+). Note that the Zero Terminal $(\mathrm{Z}+)$ of the Negative Muscle is not connected to its Max- Latch. So the Negative Muscle (M-) is not started when it arrives at Zero when it homes. The Muscle Unit can be started only by the External Start Spikes. The state of the Muscle Cells is shown in their positive maximum positions.

\subsubsection{The negative four-cell muscle unit acting alone}

If the Start Spike (SSR) starts the Negative Muscle Unit (1R-) only, the Output Arm of the Muscle Group will turn in the right $(\mathrm{CW})$ direction, as shown in Figure 6. 


\section{Four Cell Differential Muscle (Arriving at Global Max)}

Step 3+. After Homing to Zero-, the Muscle Cell ( $1 \mathrm{~L}+)$ on the Max + End remains open and the Muscle Cell (1R+) on the Zero End remains latched closed, as shown.

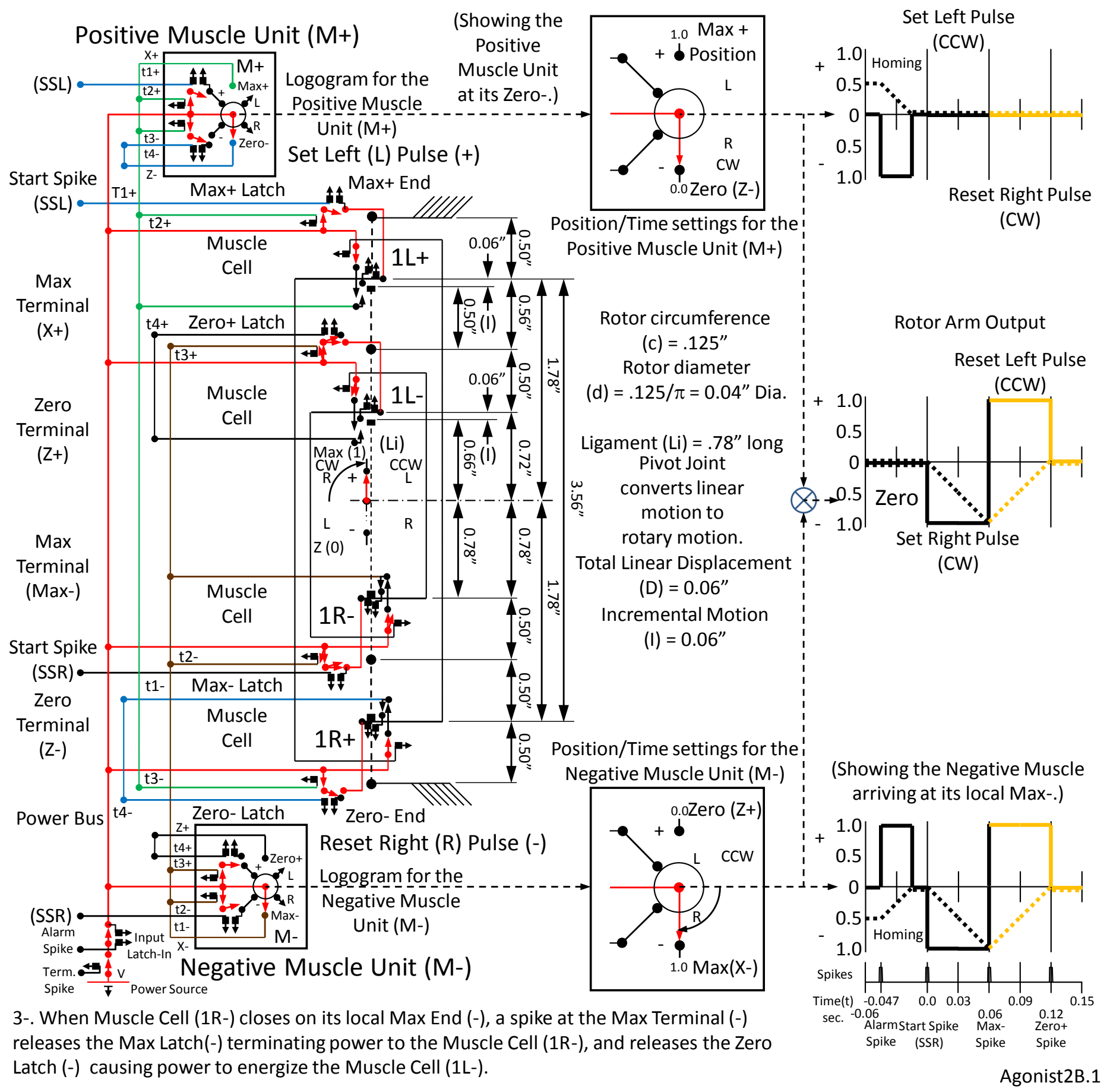

Figure 6 The symmetrical four-cell muscle group compute the sum $0+(-1)=-1$.

The states of the Muscle Cells are shown in their negative maximum positions. So the output of the symmetrical muscle is determined by which combination of positive and negative muscle units are started together. This computational ability provides the ability to avoid sensed objects, and carryout successful behavior using PWM.

\subsection{A four-cell muscle unit}

Two more muscle cells can be add to the two-cell positive and negative muscle units shown in Figure 1and Figure 2, forming the four-cell Positive Muscle Unit shown in Figure 7 and Figure 8. The addition cells increase the movement of the muscles, and increase the resolution of these movements and torque produced by the muscles for a given maximum movement.

\subsubsection{The four-cell positive muscle unit}

The four-cell Positive Muscle Unit requires two muscle cells to contract and two muscle cells to extend to obtain the full range of motion of the muscle unit, as shown in Figure 7, below. 
Positive Pulse, Four-Cell Muscle Unit (Halfway Left)

Step 9. After Muscle Cell (1 $1 \mathrm{~L}+)$ closes, the Zero Terminal (Z-) causes power to be connected to Muscle Cell (2L+). This Disconnects power to Muscle Cell $(2 \mathrm{R}+)$ and causes Muscle Cell $(2 \mathrm{~L}+)$ to start closing. This causes the Rotor Arm to start rotating left (CCW) toward the Max Position and the Muscle Cell (2R+) to start opening.

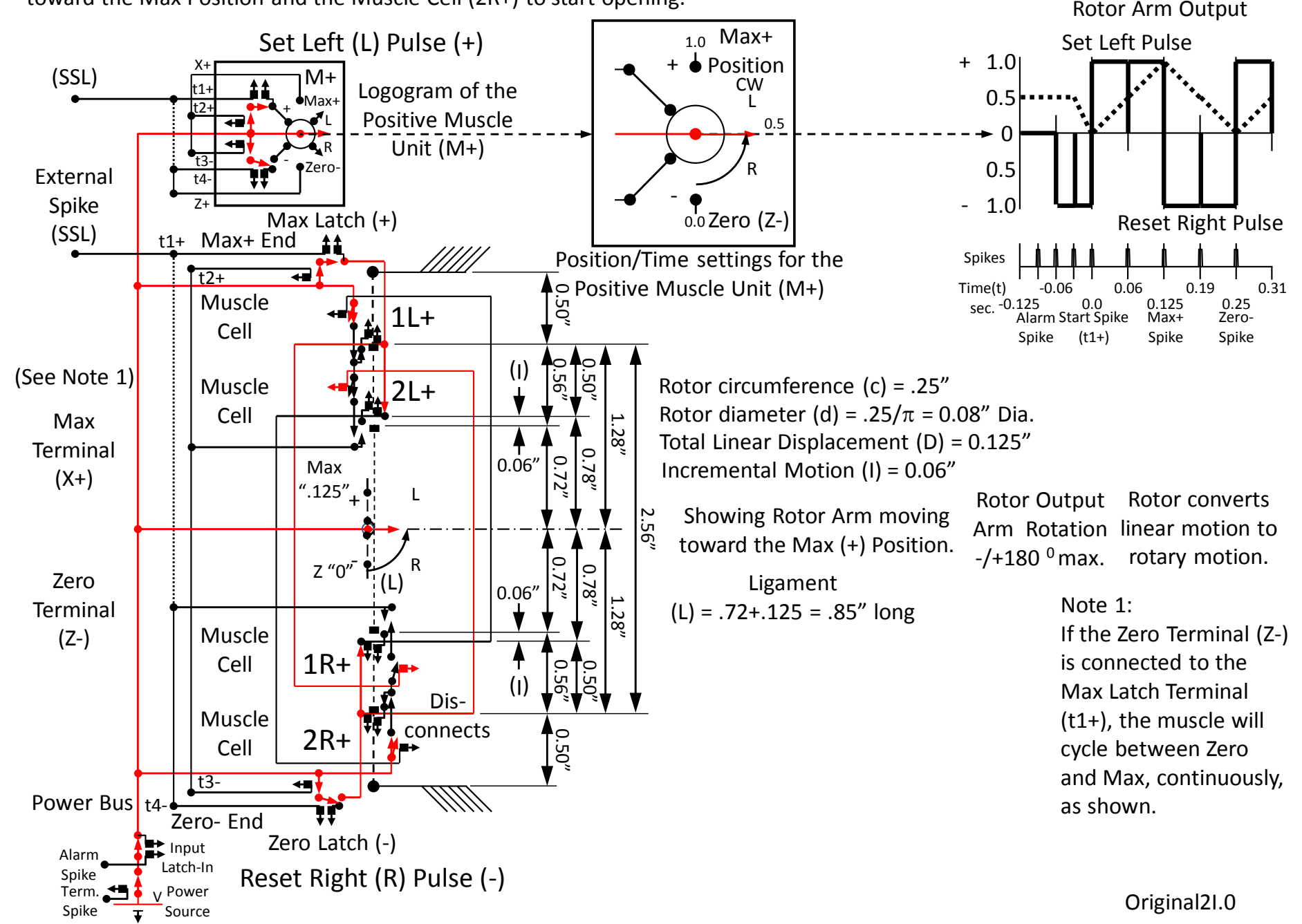

Figure 7 The positive 4-cell muscle uses a larger Rotor Arm to obtain a given range of motion. So it can produce more torque.

The four-cell muscle has a potential stopping point in the middle of its range of motion. So it has an increased position resolution. Note that a rotor diameter of 0.04 " in the two-cell muscle unit is increased to 0.08 " in the four-cell muscle unit for the same 180 degree rotation.

\subsubsection{A negative four-cell muscle unit}

The Positive Four-Cell Muscle Unit can be inverted to form the Negative Four Cells Muscle Unit shown in Figure 8. 


\section{Negative Pulse, Four-Cell Muscle Unit (Halfway Set Right)}

Step 9. After Muscle Cell (2R-) closes, power is connected to Muscle Cell (1R-). This Disconnects power to Muscle Cell (1L-) and causes Muscle Cell (1R-) to start closing. This causes the Rotor Arm to continue rotating right (CW) toward the local Max -Position causing the Muscle Cell (1L-) to start opening.

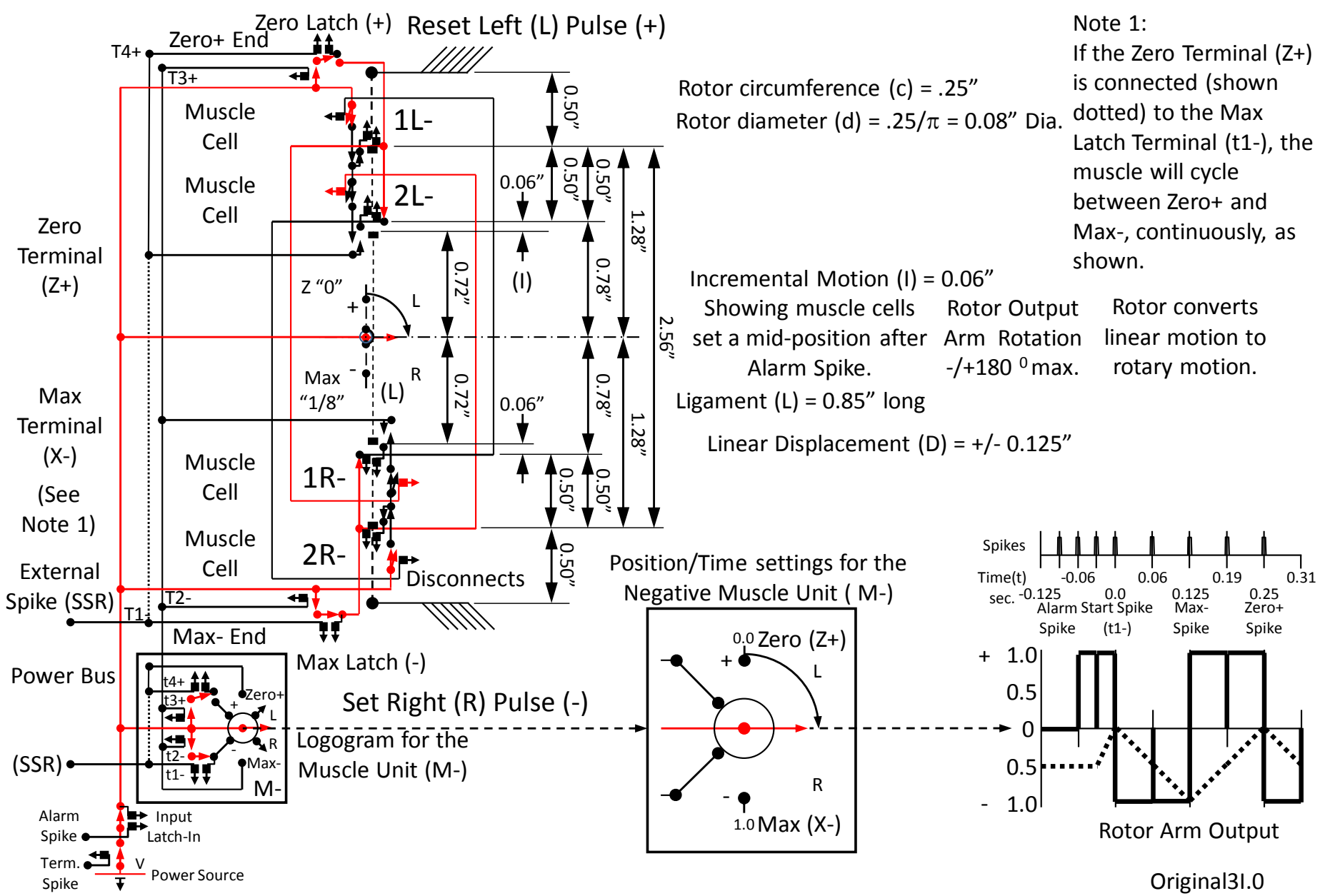

Figure 8 Since the four-cell muscle has a higher resolution over a two-cell muscle, it has the ability to store and produce more

\section{position information.}

Like the Positive Muscle Unit in Figure 7, the Negative Four Cell Muscle Unit starts to repeat its pulse train if the Zero Terminal (Z+) is connected to the Max Latch (-), which produces a second External Spike (SSR).

\subsubsection{Computing with the Eight-Cell Symmetrical Muscle Group}

The Positive Four-Cell Muscle Unit and the Negative Four-Cell Muscle Unit can be connected to a single Rotor forming an Eight-Cell Symmetrical Muscle Group, as shown in Figure 9. 
Eight-Cell Differential Muscle (Finished Homing, Both Set Muscles Started by External Start Spikes) Step 4. When the Zero Terminals are energized by the Homing Process the Zero Latches are latched in the off position.

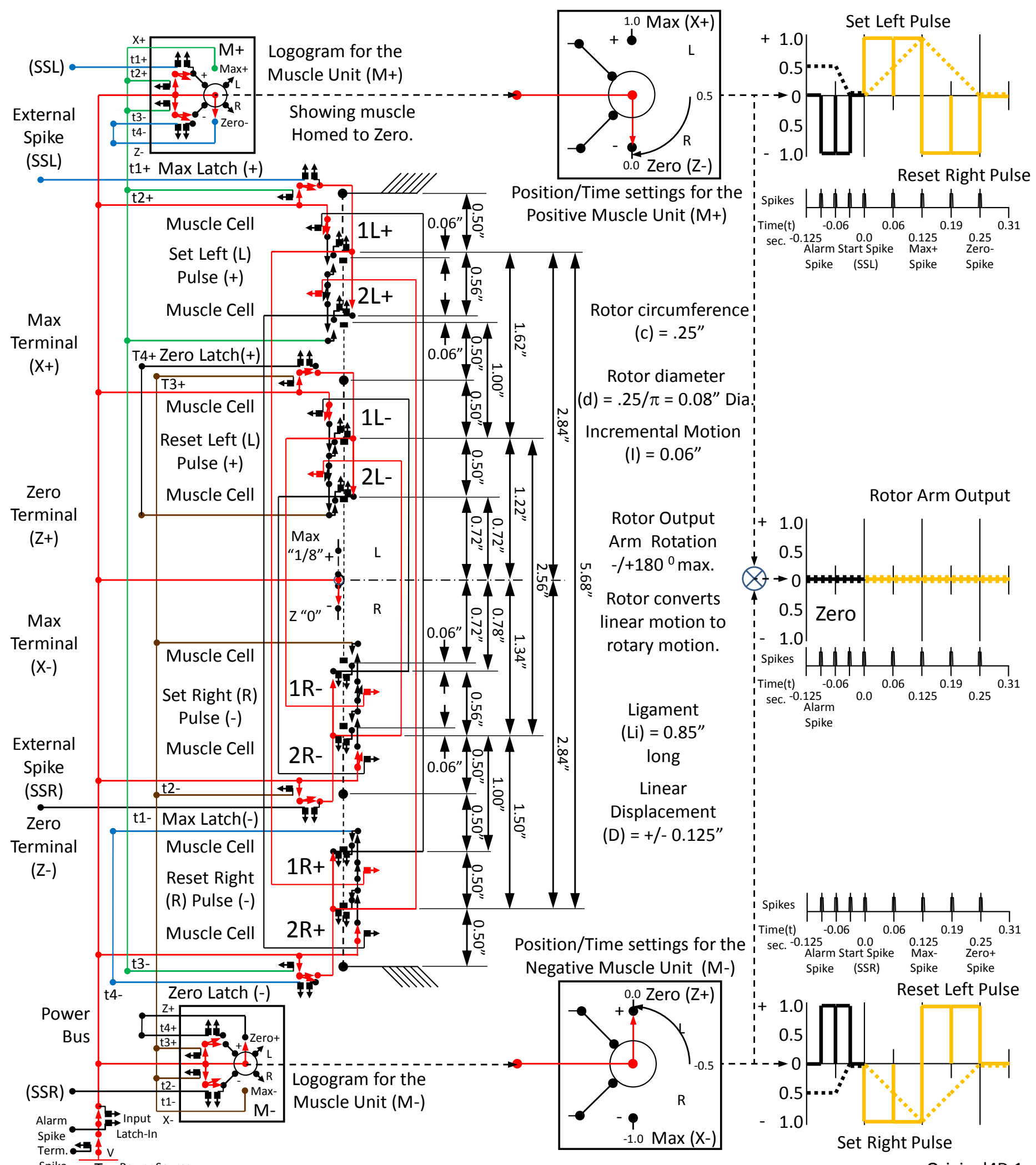

Original4D.1

Figure 9 This Symmetrical eight-cell Muscle Group shows how multiple muscle cells in both the Positive and Negative Muscle

Units are connected together to a single Rotor and Output Arm, and produce no output when both are started together.

Note that the right turning muscle cells are located on one side of the Rotor, and the left turning muscle cells are located on the other side of the Rotor. This allows the muscle to compute an output that is the sum of the active positive (left) vs. the active negative (right) turning cells. The number of different states of the multiple Muscle Cells increases the resolution of the Symmetrical Muscle. The state of the Muscles Cells is shown in their zero positions after homing.

\subsubsection{Eight-cell symmetrical muscle group with both units acting together}

When bother units are started by spikes at the Start Spike Terminals (SSR) and SSL), the Muscle Units (M+) and (M-) advance to their maximum positions, resulting with no motion of the Output Arm, as shown in Figure 10, below. 
Eight-Cell Differential Muscle (Finished Set Pulses. Resets Enabled.)

Step 6. When the Set Muscle Cells (2L+) and (1R-) finish closing in the opposite direction and the Reset Muscle Cells (2R+) \& (1L-) finish opening in the opposite direction, the Max Terminals are energized. This causes the Reset Muscle Cells (2R+) and (1L-) start closing, and Set Muscle Cells (2L+) and (1R-) to start opening, resulting in no rotation of the Rotor Arm, as shown.

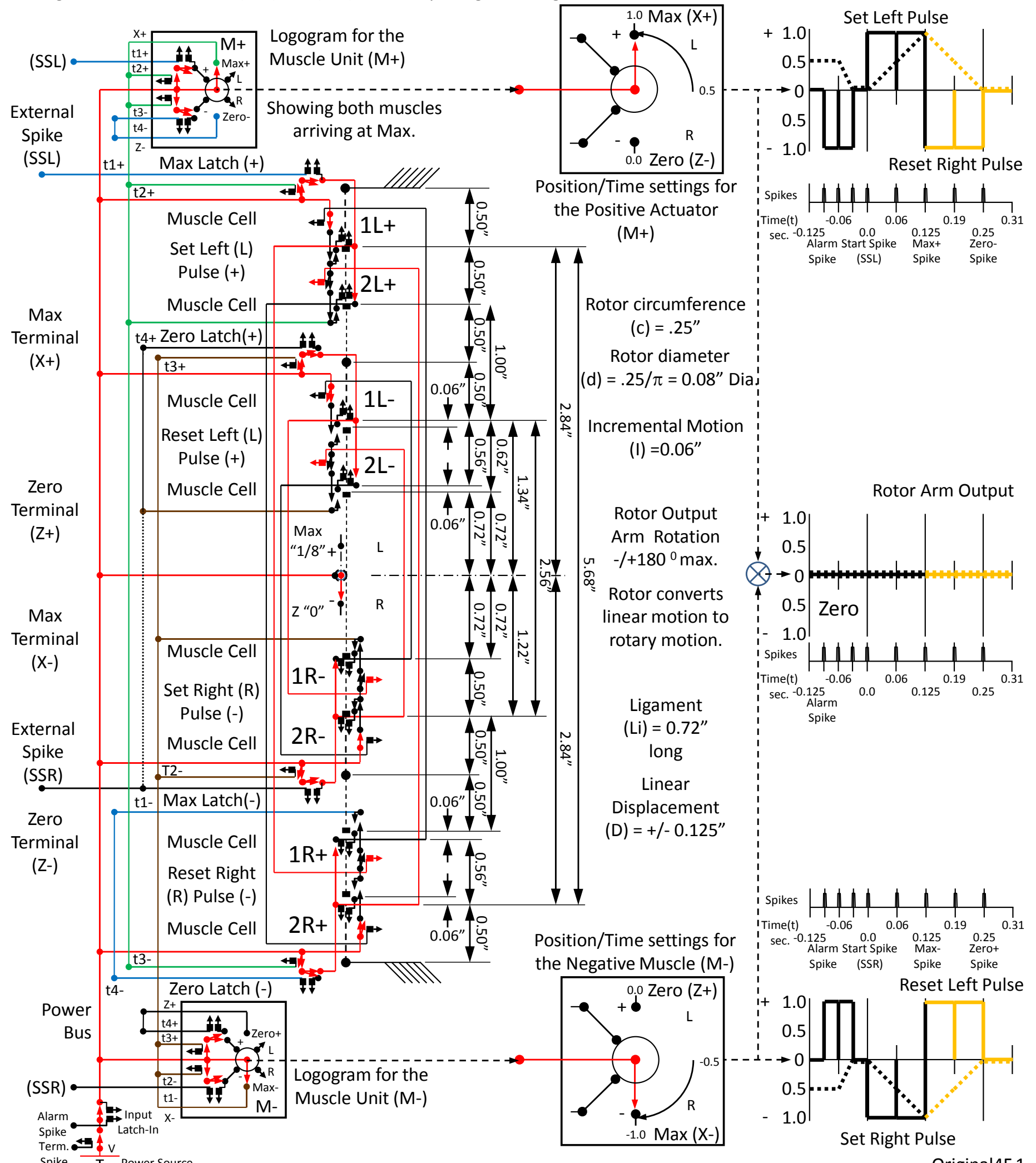

Original4F.1

Figure 10 When both Muscle Units (M+) and (M-) are started together by the External Start Spikes (SSL) and (SSR), they

reach their maximum at the same time, resulting in no motion of the Rotor Output Arm according to the equation $1+(-1)=0$.

The state of the Muscle Cells is shown in the positions of their maximum output.

\subsubsection{The eight-cell symmetrical muscle group with the positive muscle unit acting alone}

If the external Start Spike (SSL) is energized after homing, the output of the eight-cell muscle group will consists of the motion of the positive unit only, as shown in Figure 11, below. 
Eight-Cell Differential Muscle (Finished Positive Set Pulse, Reset Enabled)

Step 6. When the Set Muscle Cell (2L+) finishes closing and the Reset Muscle Cell (2R+) finish opening, the Max + Terminal is energized. This causes the Reset Muscle Cell (2R+) to start closing, and Set Muscle Cell ( $2 \mathrm{~L}+)$ to start opening, resulting in a Reset rotation of the Rotor Arm, as shown.

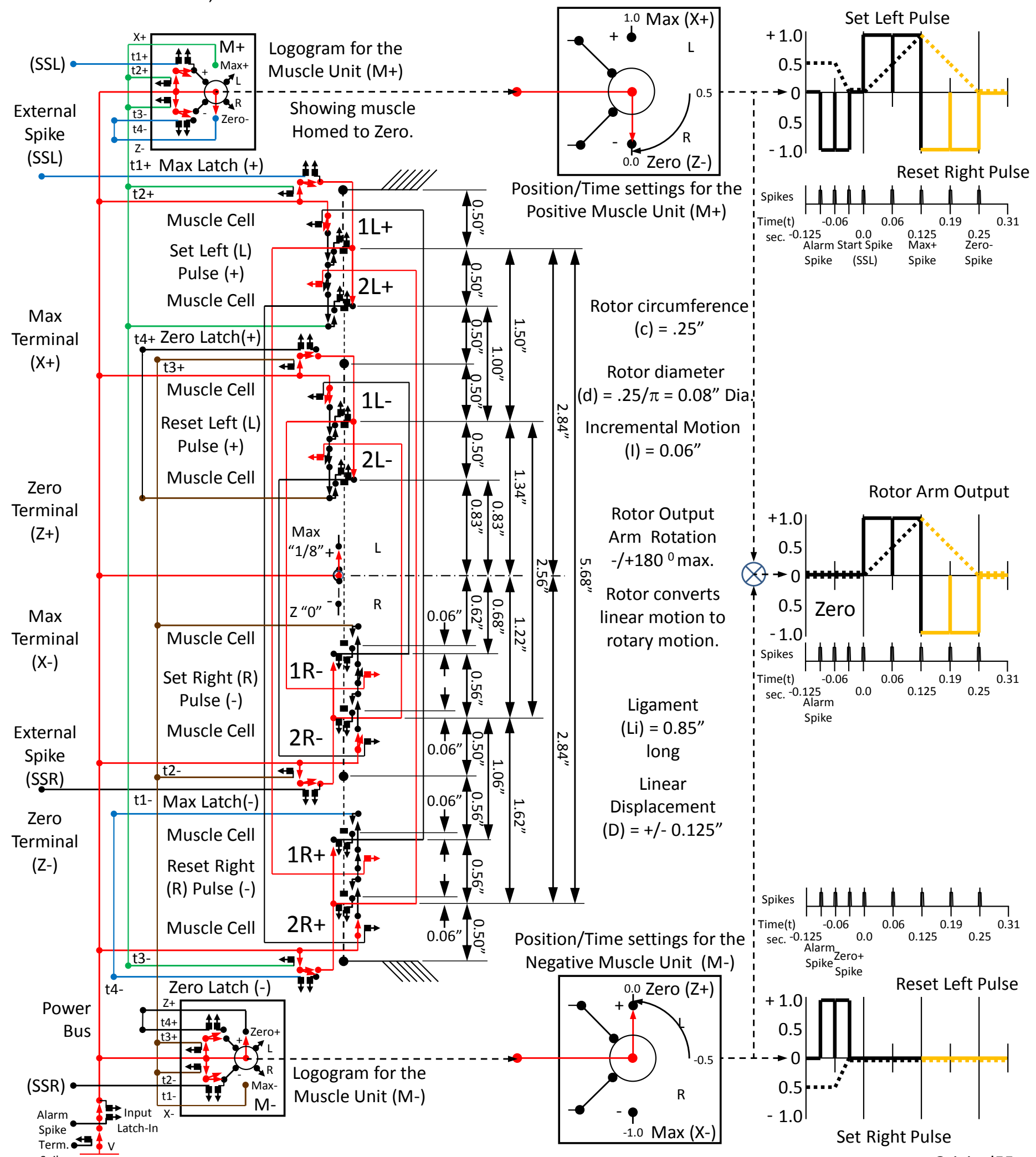

Original5F

Figure 11 The Rotor Arm Output of the eight-cell symmetrical muscle group is produced by the motion of the positive muscle unit according to the equation $1+0=1$.

If the muscle is stopped before 006 seconds, the output motion will be just .5 units.

\subsubsection{The eight-cell symmetrical muscle unit is shown with the negative muscle unit acting alone.}

When the negative muscle is started by a spike at the External Start Spike Terminal (SSR), the Rotor Output Arm moves clockwise (right) according to the movement of the negative muscle (M-). As shown in Figure 12, below. 
Eight-Cell Differential Muscle (Finished Homing, Both Set Muscles Started by External Start Spikes) Step 6. When the Set Muscle Cell (1R-) finishes closing and the Reset Muscle Cell (1L-) finish opening, the Max - Terminal is energized. This causes the Reset Muscle Cell (1L-) to start closing, and Set Muscle Cell (1R-) to start opening, resulting in a Reset rotation of the Rotor Arm, as shown.

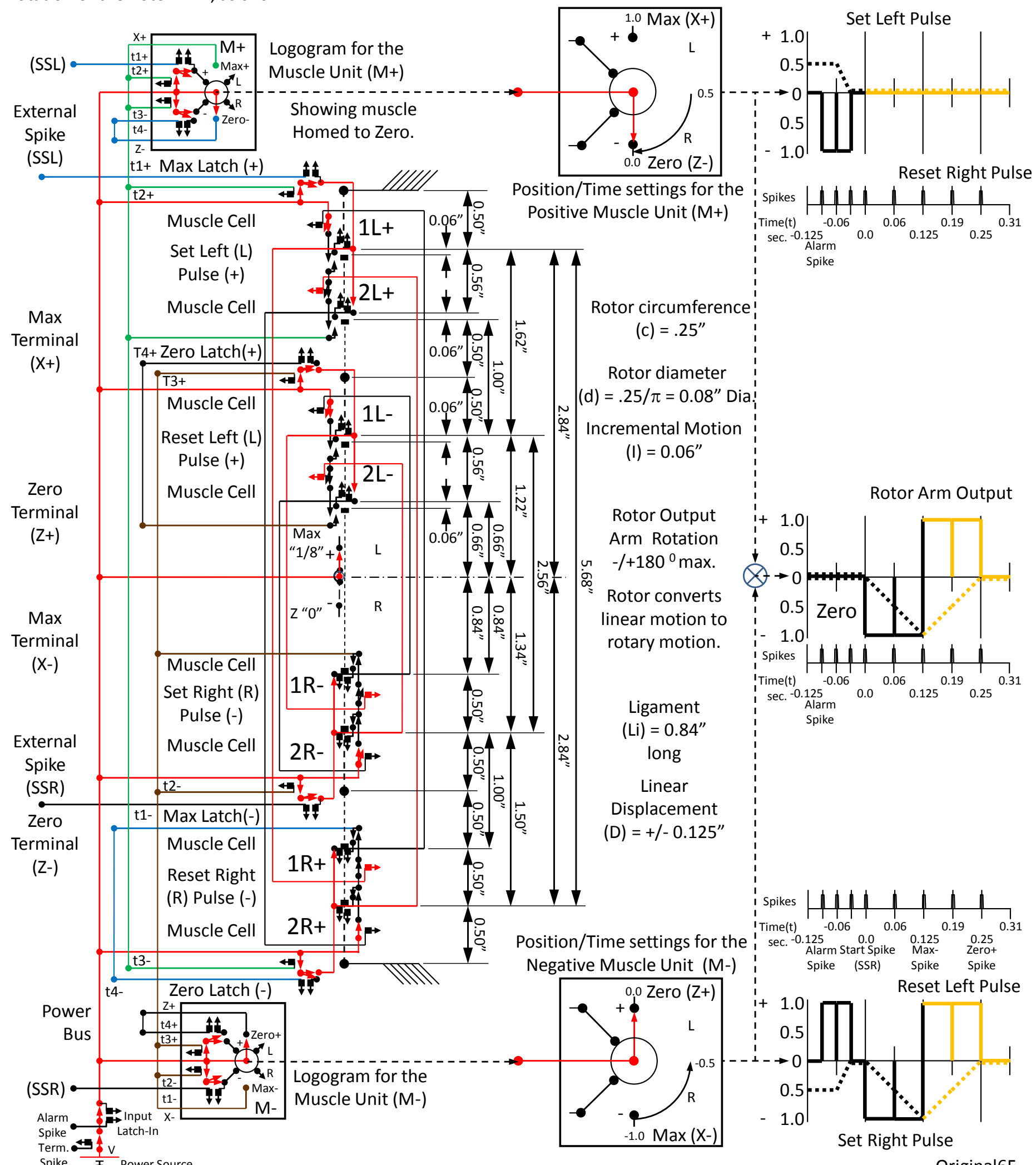

Original6F

Figure 12 When the Negative eight-cell Muscle Unit is started by the External Start Spike Terminal (SSR) alone, the output of

the symmetrical muscle group is determined by the Negative Muscle unit according to the equation $0+(-1)=-1$.

Since there are two stopping states, the output could be -0.5 if power to the Negative muscle is interrupted before it reaches -1 . The number of stopping positions increases as the number of muscle cells in each Muscle Unit is increased.

\subsection{A sixteen-cell muscle Unit}

The number of cells in the muscle unit can be further increased to provide greater movement or increased velocity, increased resolution, and a greater force of rotation (torque) if the displacement of the muscle is reduced by increasing the radius of the rotor. A sixteen-cell muscle unit requires eight muscle cells in each Positive and Negative Muscle Unit. This provides adequate resolution to demonstrate the operation of the muscles used in most of this paper.

\subsubsection{Sixteen-Cell Positive Muscle Unit}


The sixteen-cell positive muscle unit is shown below in Figure 13 with its muscle cells in the states after homing to zero.

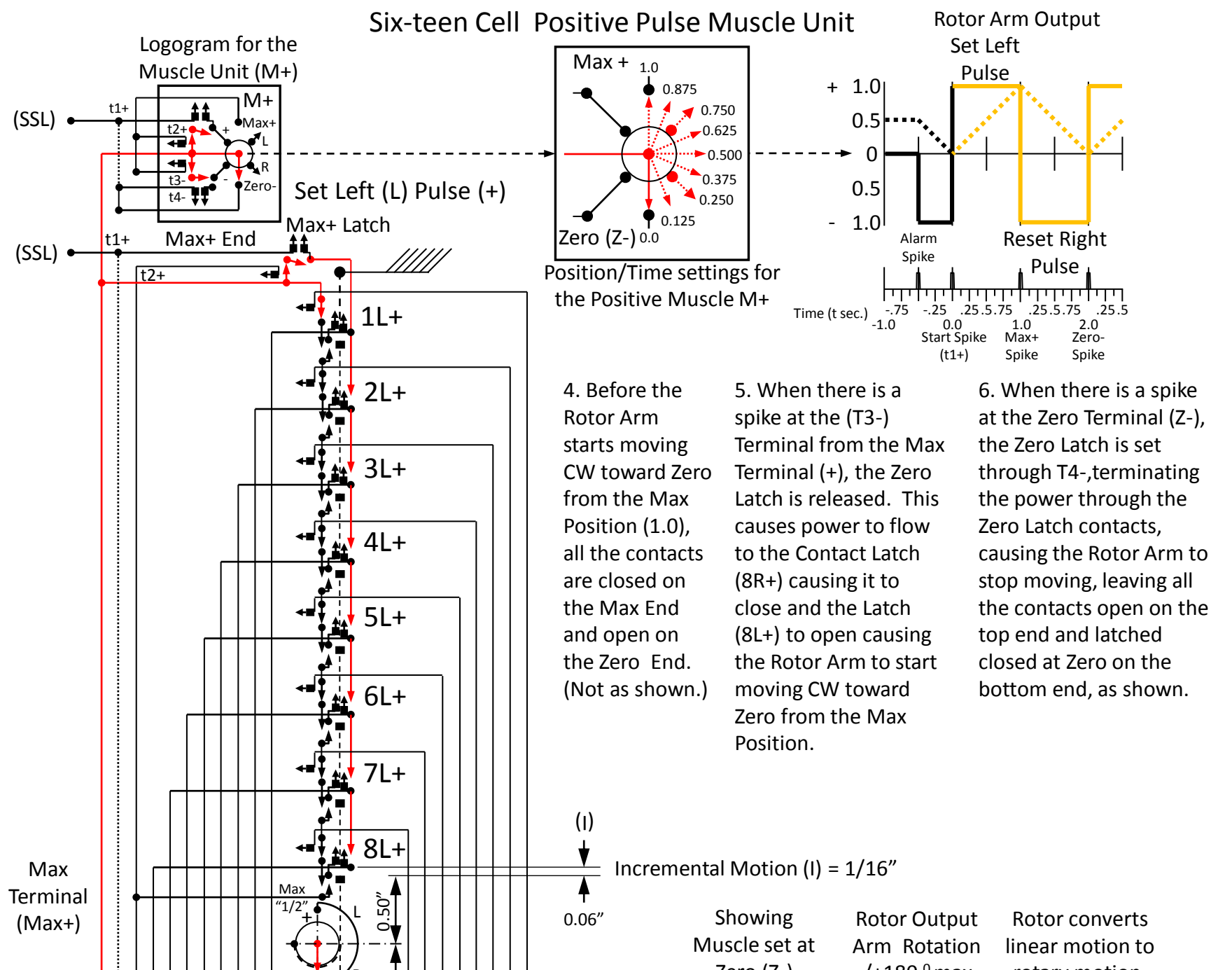

Zero (Z-) $\quad-/+180^{\circ}$ max. rotary motion.

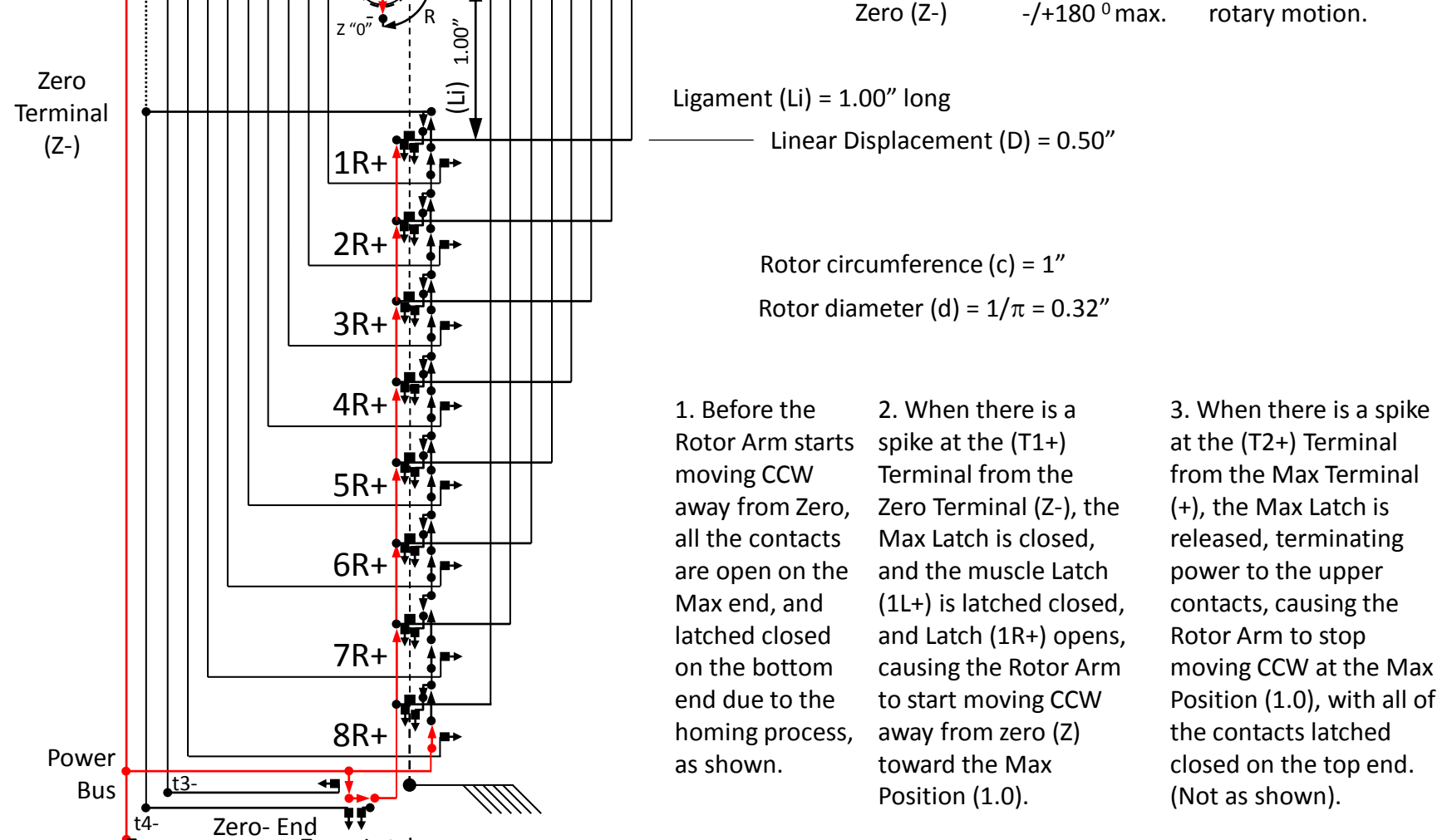

MuscleA.1

Figure 13 In the Sixteen-Cell Positive Muscle Unit, the diameter of the Rotor can be increased to 0.32" for a 180 degree rotation because there are more Muscle Cells. This results in a higher motion resolution and increased torque using the same kind of muscle cells that have been used before.

The muscle produces a series of positive and negative pulses, each with a pulse (time) width of eight units. Each time unit is equal to the time it takes for a single muscle cell to close and its antagonistic mate to open.

\subsubsection{Sixteen-Cell Negative Muscle Unit}


The Sixteen-Cell Negative Muscle Unit (M-) is the same as the Sixteen-Cell Positive Muscle Unit (M+) except it is inverted. So it starts (sets) down instead of up, as shown in Figure 14. The Muscle Cells are shown in the state that produces zero output position.

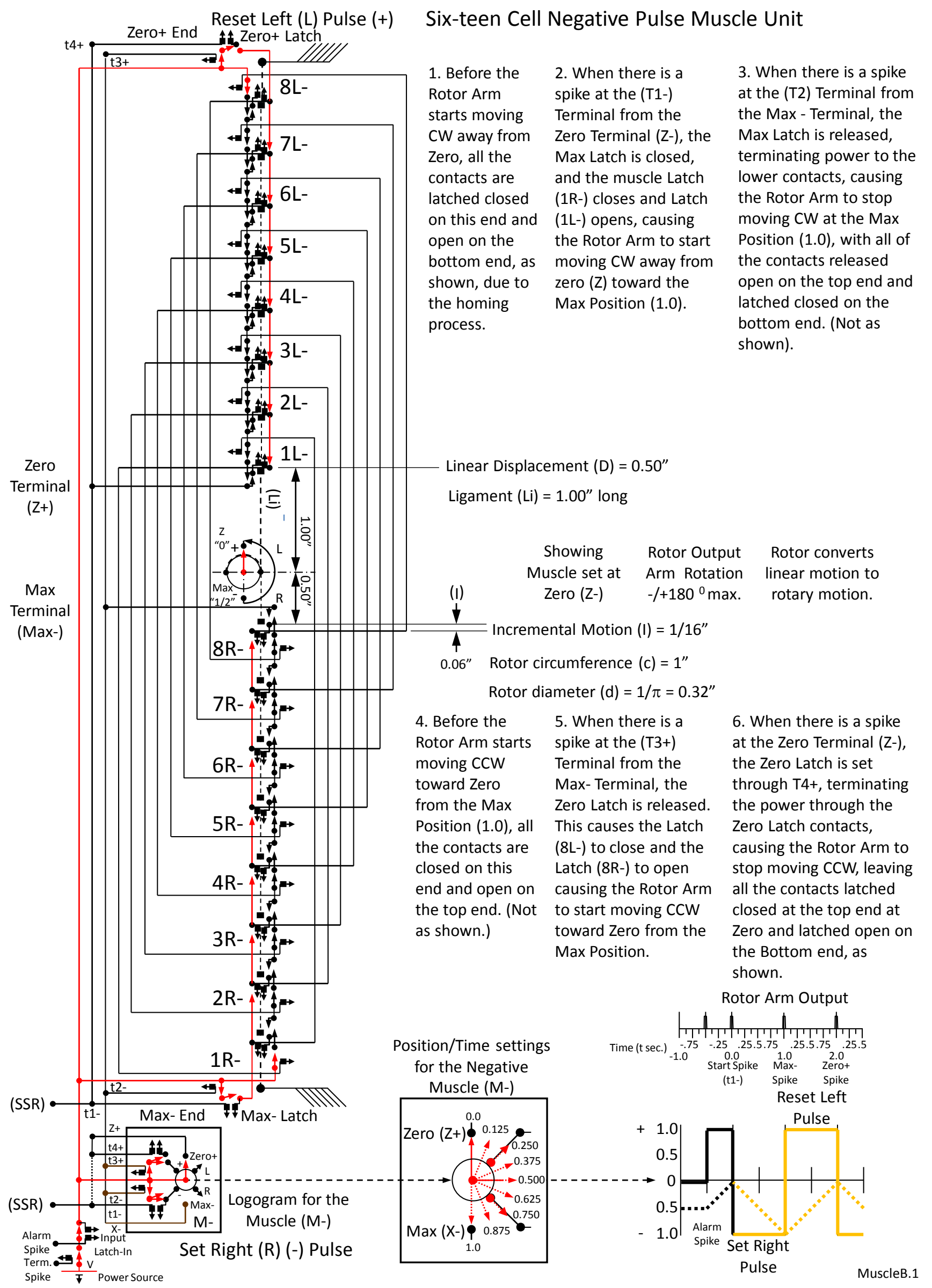

Figure 14 In the sixteen-cell negative muscle unit, the Max Terminal is under the Zero Terminal.

With so many cells acting their own rate, the output speed of these muscles may not be uniform. So the action of the cells in multiple muscles can by synchronized by a single High Frequency Spike Train (HFST) from a single Spike Generator. This causes the rate of contraction and extension of the muscles to be determined by one timer rather than the individual cells within the muscle, and allows the motion of multiple muscles to act in synchronism. 


\subsubsection{Driving a Positive 16 Cell Muscle Unit with a Spike Generator}

The Positive Muscle Unit (M+) in Figure 1 can be can be used as a High Frequency Spike Generator (SG) that can be connect to the sixteen cell muscle in Figure 13, as shown in Figure 15.

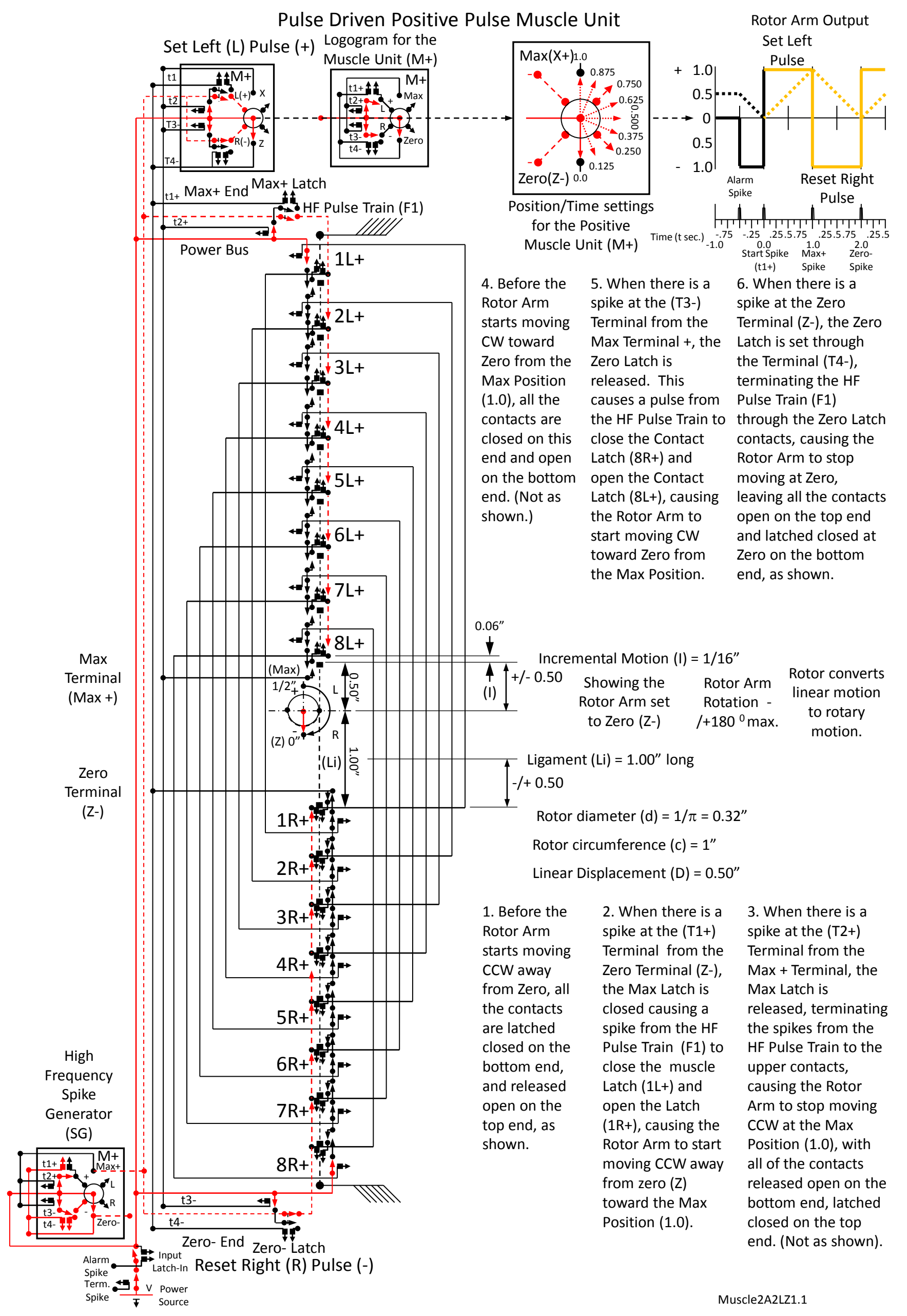

Figure 15 Signals from the High Frequency Spike Generator create a High Frequency Spike Train (HFST) that energizes each

muscle cell instead of each muscle cell being powered by the Power Bus directly. This means that the muscle movement is paced by the (HFST).

The spike driven muscle operates as evenly as the HFST input rather than being driven at the speed of each 
individual muscle cell.

\subsubsection{Driving a Negative 16-Cell Muscle Unit}

The High Frequency Spike Generator can be connected to the sixteen-cell negative muscle unit forming the negative spike driven muscle unit shown in Figure 16.

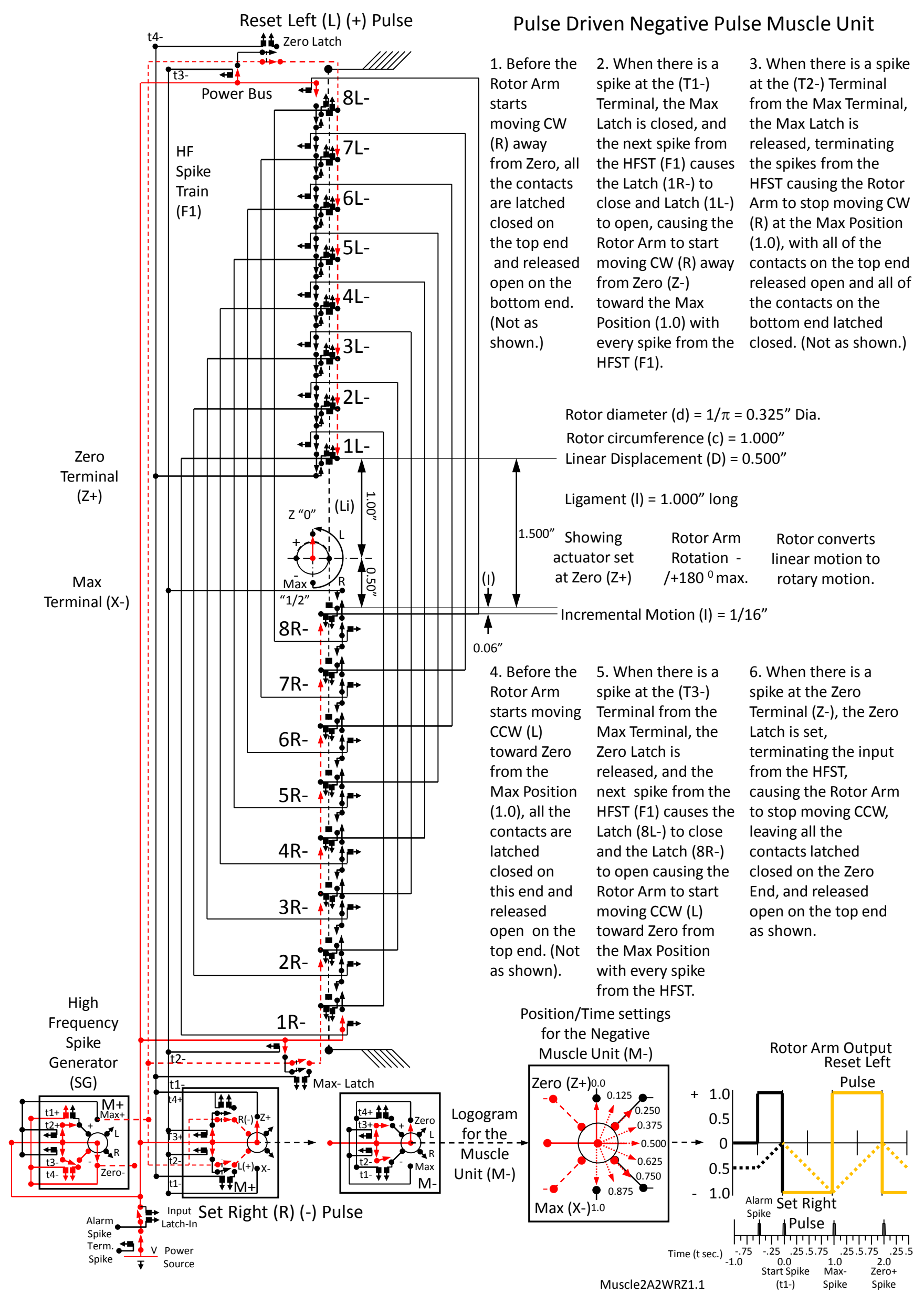

Figure 16 The spike driven 16-Cell Negative Muscle Unit starts right (clockwise) and runs in sync with the High Frequency

Spike Generator.

Note that the Rotor Output Arm in the Negative Muscle Unit shown above in Figure 16 turns clockwise from zero, and the Rotor Output Arm of the Positive Muscle Unit turns in the opposite counterclockwise direction from zero, as 
Pulse width modulation

shown in Figure 15.

\subsubsection{Connecting positive and negative muscles forming a 32 cell muscle group}

Positive and negative muscle units can be connected together in series so that their motions are added as if they were connected by means of a differential, forming the 32-cell symmetrical muscle shown in Figure 17.

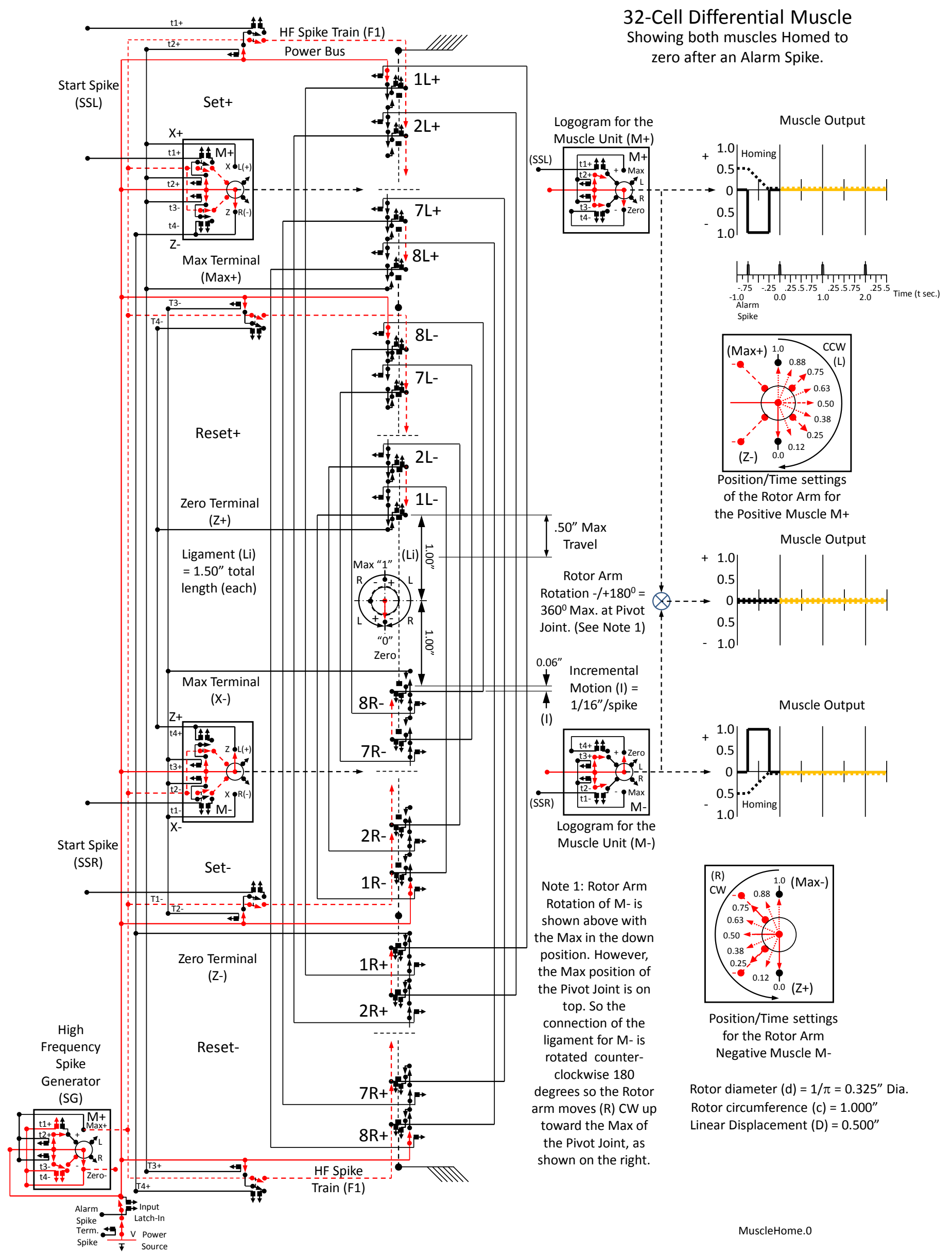

Figure 17 The positive and negative muscles units are connected to a single rotor with their left turning cells connected to the

top end of the rotor and their right turning cells connected to the bottom end of the rotor, forming a 32 cell symmetrical

muscle group. After homing the Muscle Group computes $0+0=0$

Four of the eight muscle cells, 3, 4, 5 and 6 , in each muscle unit are left out because of the limitation of the page 
size. All of the Muscle Cells in Figure 17 above are shown at the zero muscle output state because they have been homed by an Alarm Spike.

\subsubsection{A 32-cell muscle group with both muscle units activated}

Since motions of both muscle units are created by the single High Speed Spike Train, they are able to add or cancel each other precisely. So when both muscles units operate at the same time there is no output motion because the set right motions are cancelled by the set left motions, as shown in Figure 18.

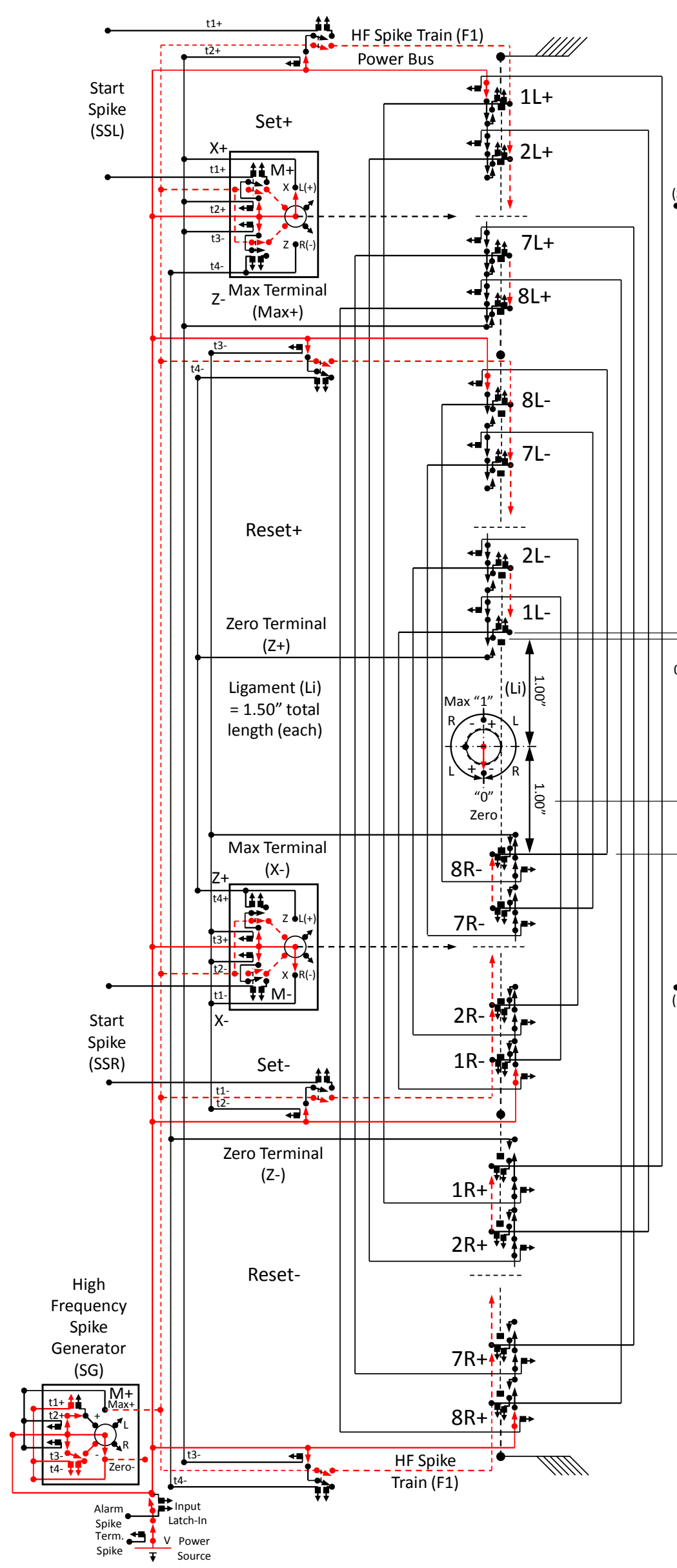

32-Cell Differential Muscle Showing both muscles at their maximum position after starting from zero at the same time from spikes at (SSL) and (SSR).

Logogram for the Muscle Unit (M+)

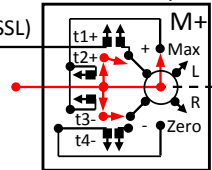

Incremental

Motion (I) = $1 / 16$ "/spike

\section{(I)} $\overbrace{}^{4}$ Muscle Output
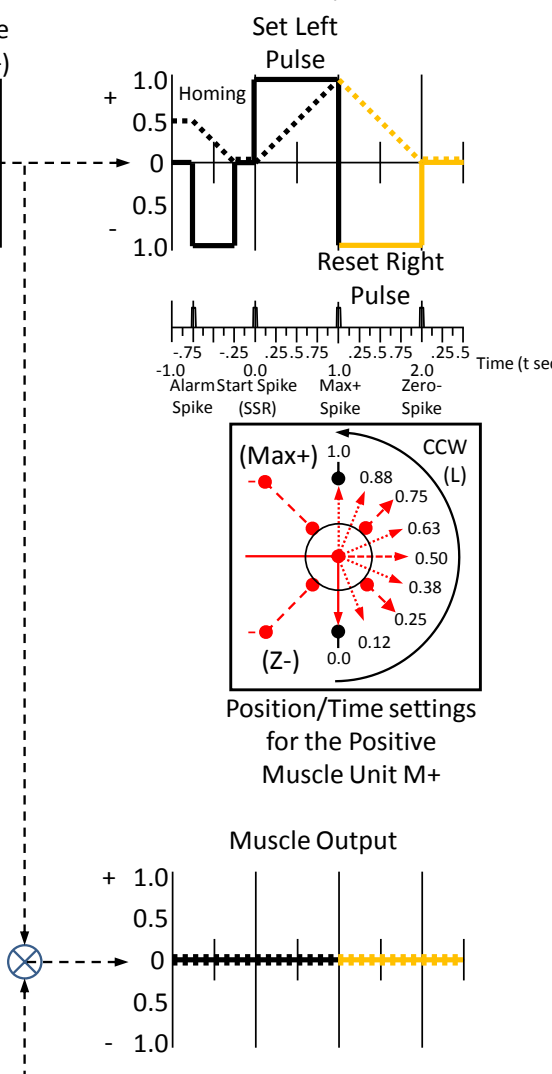
Rotation $-1+180^{\circ}$

$360^{\circ}$ Max at Pivot Joint. (See Note 1)

$-1.0$ Travel
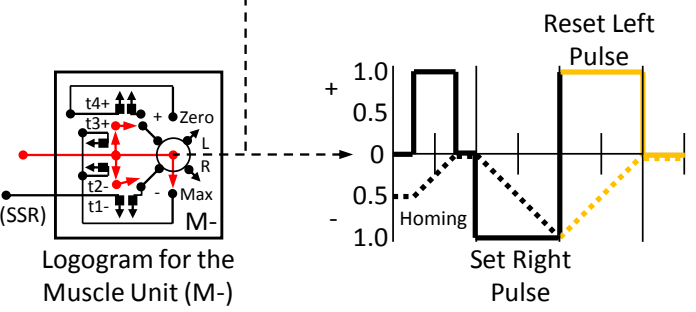

Note 1: Rotor Arm Rotation of $\mathrm{M}$ - is shown above with the Max in the down position. However, the Max position of the Pivot Joint is on top. So the connection of the ligament for $\mathrm{M}$ - is rotated counterclockwise 180 degrees so the Rotor arm moves (R) CW up toward the Max of the Pivot Joint, as shown on the right.

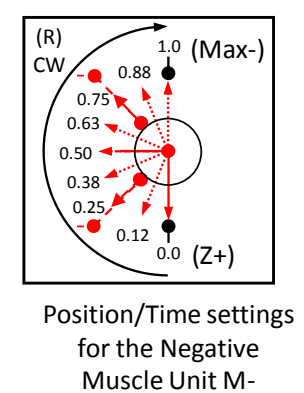

Rotor diameter $(d)=1 / \pi=0.325^{\prime \prime}$ Dia. Rotor circumference $(c)=1.000^{\prime \prime}$ Linear Displacement $(D)=0.500^{\prime \prime}$

MuscleZeroDiff.2

Figure 18 When both Muscle Units (M+) and (M-) are started at the same time by spikes at the External Start Spike

Terminals after Homing, the motion of each Muscle Unit cancels the motion of the other, resulting in no output motion of the 
Muscle Group according to the equation $1+(-1)=0$.

This ability of Muscle Units to cancel the motion of other Muscle Units in a Muscle Group is the foundation of biological computation using time extents.

\subsubsection{A 32-cell muscle group with the Positive Unit activated}

When the top Positive Muscle Unit (M+) is activated by the Start Spike Terminal (SSL) only, the Symmetrical Muscle produces the output of the top Muscle Unit, only, as shown in Figure 19.

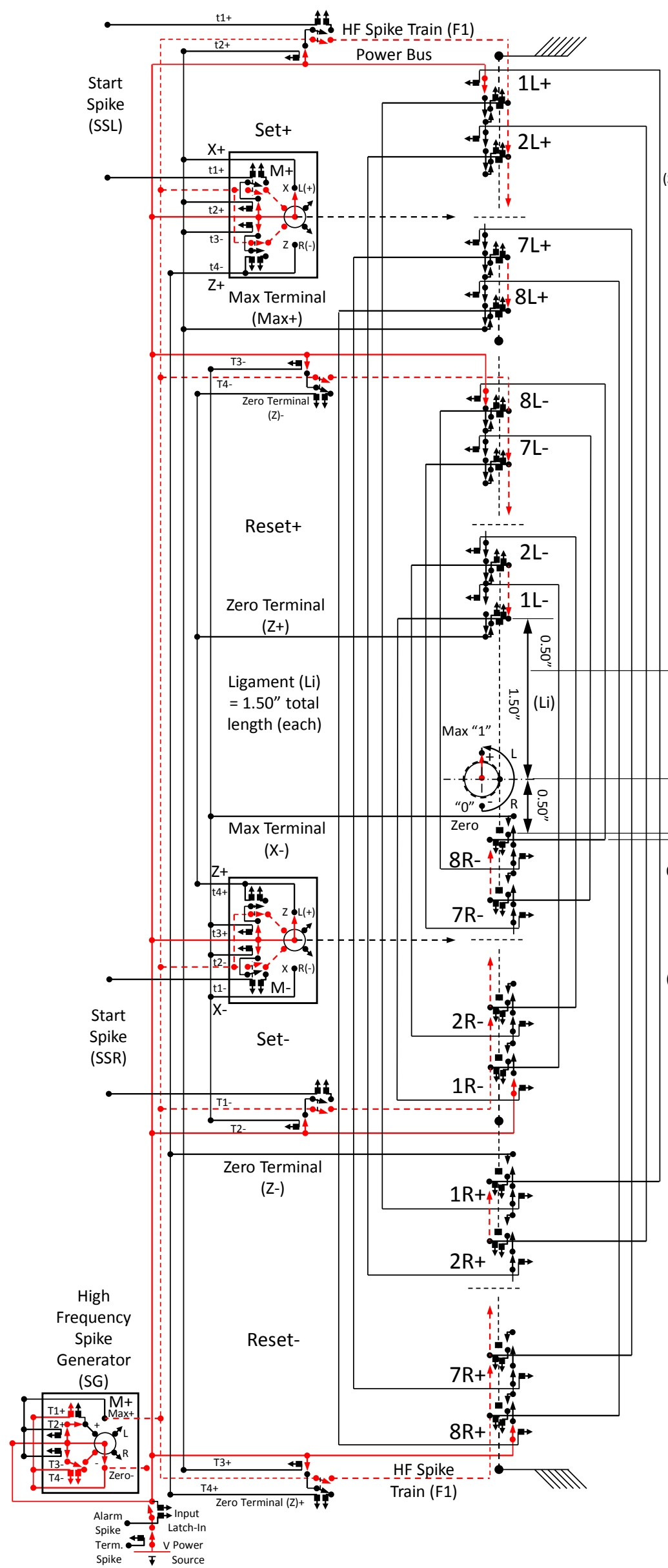

32 Cell Differential Muscle Showing only muscle $(\mathrm{M}+)$ at its maximum position after homing to zero and a spike at (SSL).

Logogram for the Muscle Unit (M+) Muscle Output

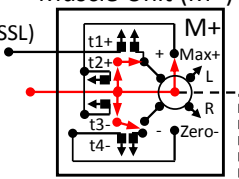

Rotor circumference

(c) $=1^{\prime \prime}$

Rotor diameter (d) $=1 / \pi=0.32^{\prime \prime}$

Linear Displacement (D) $=+/-0.500^{\prime \prime}$

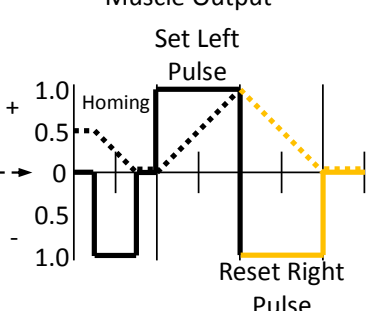
$\prod_{-.75}\left\|_{-25}\right\|_{0.25 .5 .75}^{\text {Pulse }}$

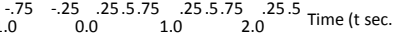

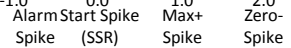

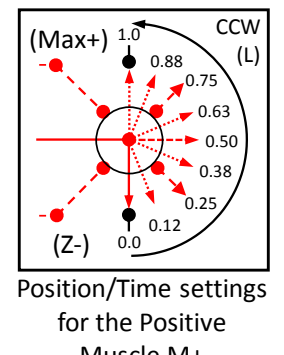

Muscle $\mathrm{M}+$

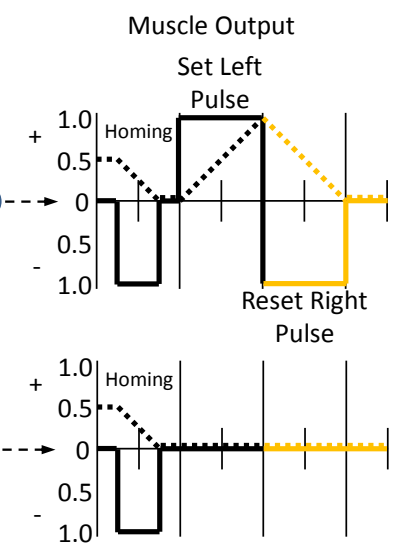

Logogram for the Muscle Unit (M-) Incremental Motion (I) $=1 / 16^{\prime \prime} /$ spike

Note 1: Rotor Arm Rotation of M- is shown here with the Max in the down position. However the Max position of the Pivot Joint is on top. So the connection of the ligament for $\mathrm{M}$ - is rotated counterclockwise 180 degrees so the Rotor arm moves ( $R$ ) CW up toward the Max of the Pivot Joint, as shown.

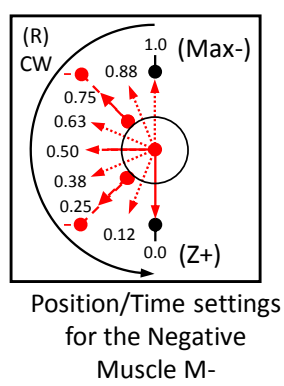

Figure 19 In this symmetrical muscle group, where only the Positive Muscle Unit is started, the set of antagonistic muscle units produce an output that is the sum of the output of each muscle unit according to the equation $1+0=1$. 
The state of the muscle cells when the Positive Muscle is at its maximum is shown above with all of the top (left turning) Muscle Cells (L) closed, and all of the bottom (right turning) Muscle Cells (R) open.

\subsubsection{2-cell muscle group with the Negative Unit activated}

When the Bottom Muscle Unit (M-) is activated, the output of that Symmetrical Muscle is produced by the Bottom

Muscle (M-), as shown in Figure 20.

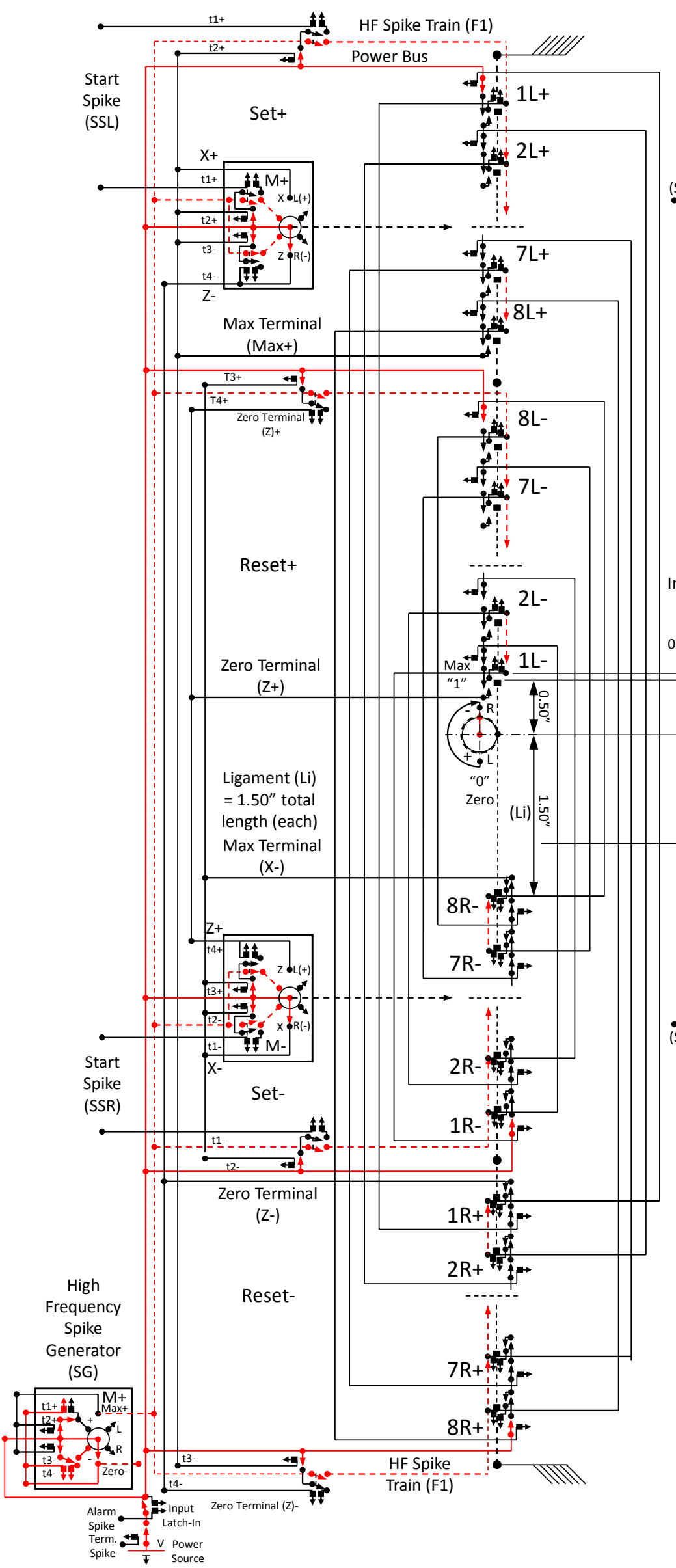

\section{Cell Differential Muscle}

Showing only one muscles (M-) at its maximum position starting from zero by a spike at (SSR) after homing.

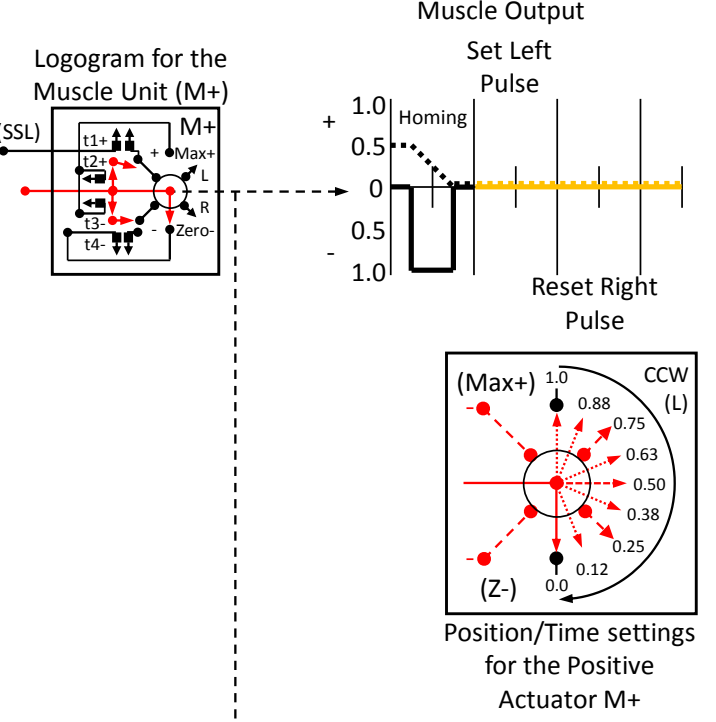

Rotor Arm Output (I) $=1 / 16^{\prime \prime} /$ spike $06^{\prime \prime}$

Rotor Arm (i) Rotation $-/+180^{\circ}$ $=360^{\circ}$ Max. at Note 1) 1" Max Travel
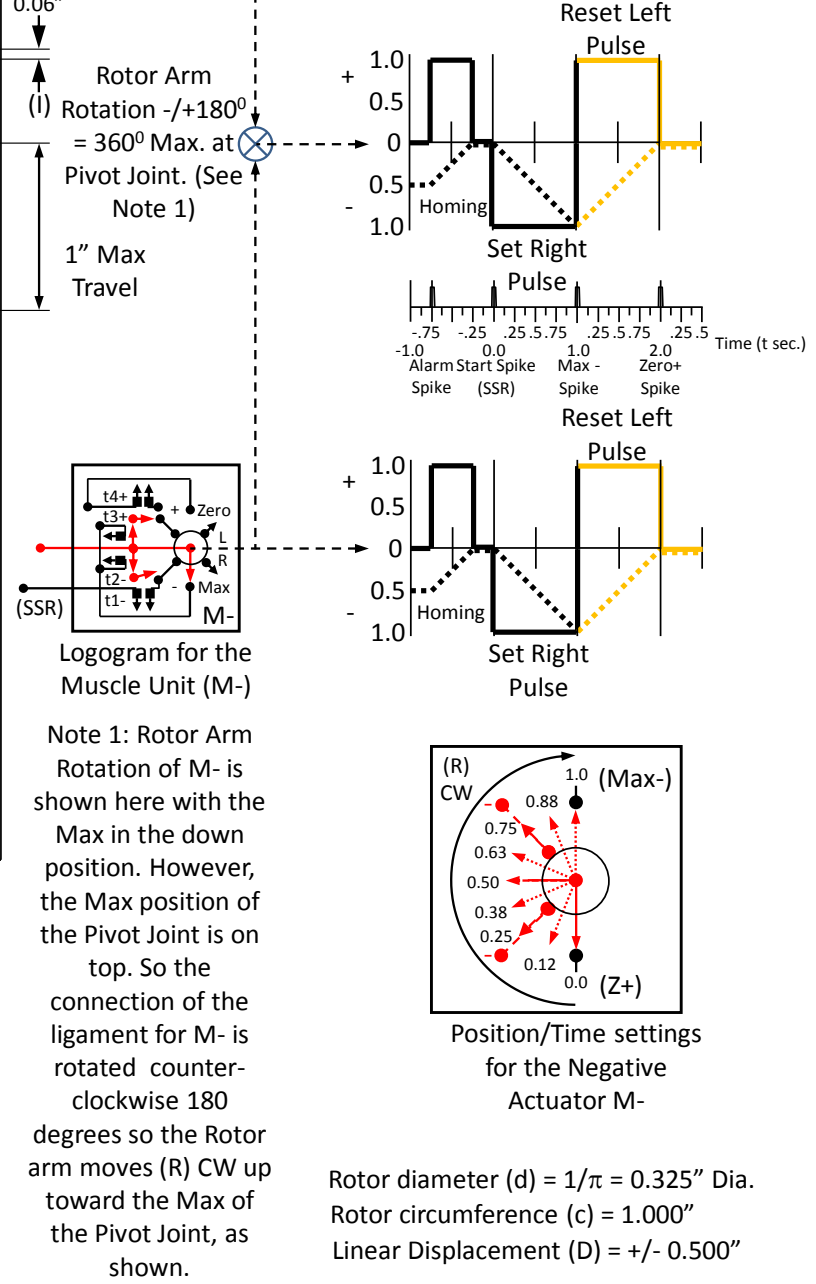
Actuator M-

Rotor diameter $(\mathrm{d})=1 / \pi=0.325^{\prime \prime}$ Dia. Rotor circumference $(c)=1.000^{\prime \prime}$ Linear Displacement $(D)=+/-0.500^{\prime \prime}$

Figure 20 In the symmetrical muscle group, the muscle unit that is not activated just rides up and down with the motion produced by the active muscle unit without influencing the output motion in the computation $0+(-1)=-1$.

In this case all bottom (right turning) Muscle Cells are closed, and all top (left turning) Muscle Cells are open, 
Pulse width modulation

showing the state of the Muscle Cells when the bottom muscle is at its maximum position. The inactive muscle cells just act as tendons that ride up and down due to the action of the active muscles. This kind of symmetrical muscle group provides the computations needed to control the motion of an animal.

\subsection{Motion Sequence and object avoidance using Pulse Width Modulation}

The muscles shown so far act just as oscillators. In order to control the timing and sequence of their movements it is necessary to break up time into repeating periods, and start and stop the muscles at some time within these periods. These periods can be created by using a Frequency Divider (FD) to count some number of spikes from a High Frequency Spike Generator (SG), which then produces an output spike that delineates the end of a period and the beginning of a new period. This timing framework is the basis of the carrier frequency used in Pulse Width Modulation (PWM).

\subsubsection{A frequency divider unit}

The Frequency Divider requires one or more Frequency Divider Units (FDU) shown on the following circuit diagram shown in Figure 21.

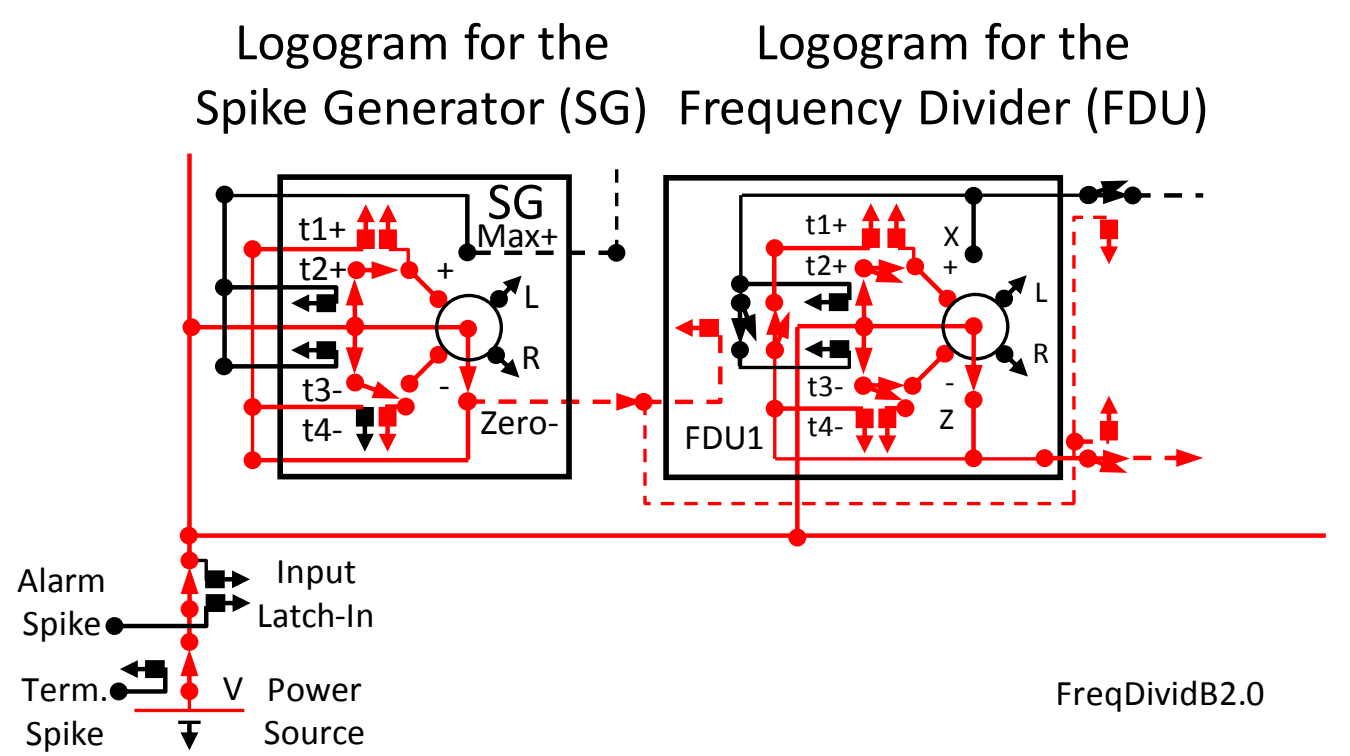

Figure 21 A Frequency Divider Unit (FDU) produces an output spike and resets itself only when it's Input Switch is closed.

The Frequency Divider Unit (FDU) is essentially the same as the Spike Generator (SG), except the connection from Zero (Z) to the Start Terminal (t1+) in the Frequency Divider Unit (FDU) has to be switched closed by the output of the Spike Generator (SG). And the connection from the Max Terminal (X+) to the Reset Terminal (t3-) in the Frequency Divider Unit (FDU) has to be switched closed by the next output spike from the Spike Generator (SG). Thus, the Pulse Input Switch in the (FDU) is closed on every other output spike from the Spike Generator (SG). So the Frequency Divider Unit (FDU) skips every other pulse from the Frequency Generator since the second pulse from the Frequency Generator resets the FDU allowing the third pulse to causes the FDU to produce an output set pulse at that time.

The Frequency Divider Unit acts like a Frequency Generator that operates at half of the input frequency, as shown in Figure 22, below.

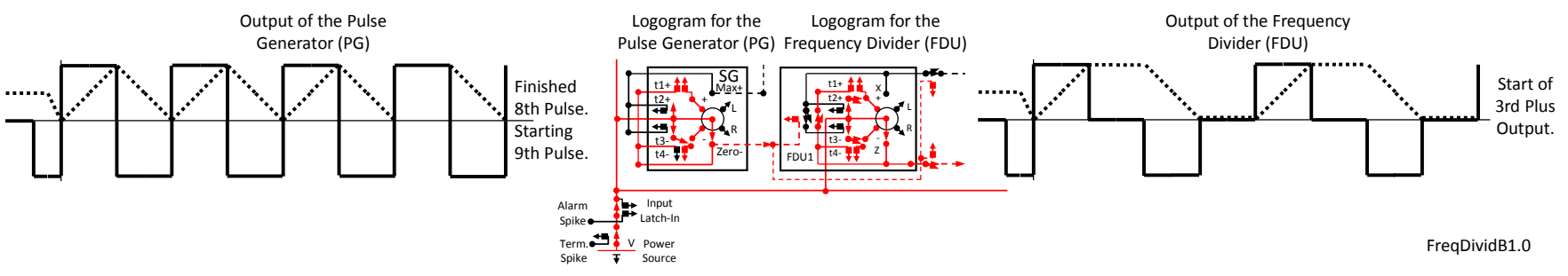


Pulse width modulation

Figure 22 The Frequency Divider Unit produces one half the spike frequency of the input spike frequency.

The FDU can be connected to another FDU that continues to divide the input spike frequency, creating periods consisting of eight spike between outputs at (T1) and (T2) forming the Frequency Divider (FD) shown in Figure 23, below.

\section{8 to 1 Period Spike Driven Frequency Divider}

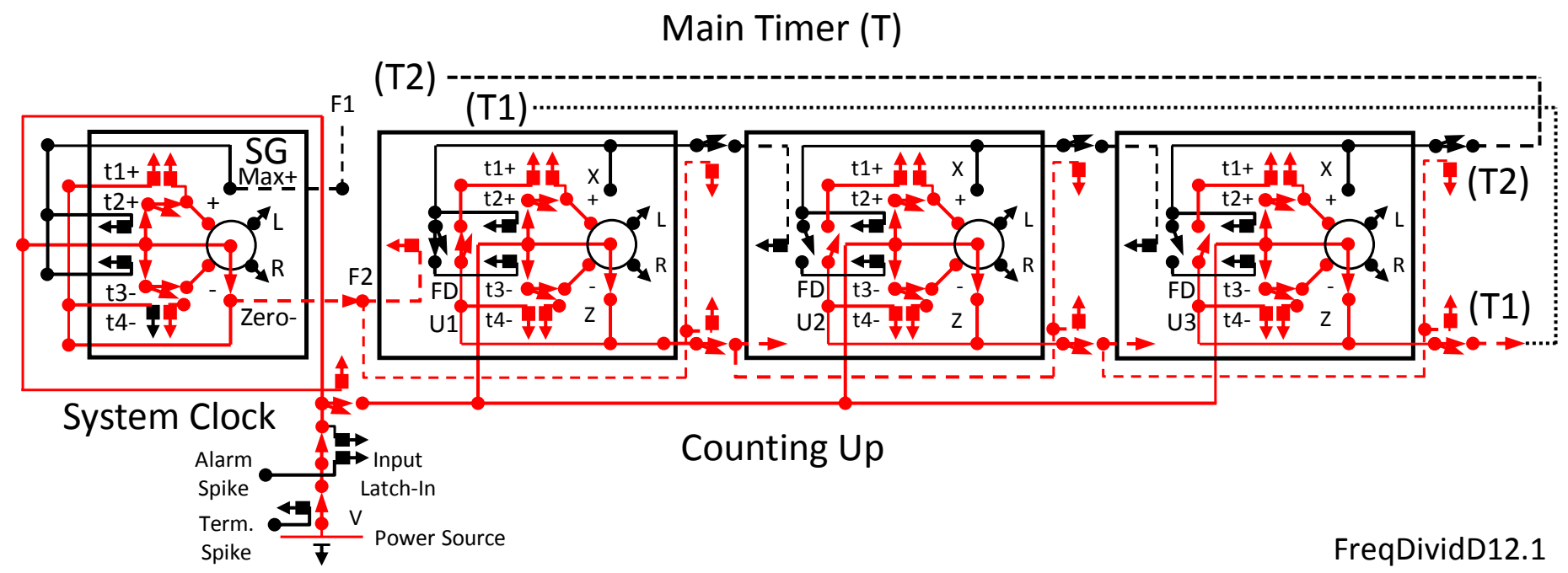

Figure 23 The Spike generator (SG) and Frequency Divider (FD) form a Main Timer (T). The frequency reduction is equal to $1 / 2^{n}$, where $n=$ the number of FDU's.

Frequency dividers are effective in producing an output after many input spikes. For example, the seven (7) unit frequency divider shown in Figure 24, below, can produce an output after 128 input spikes.

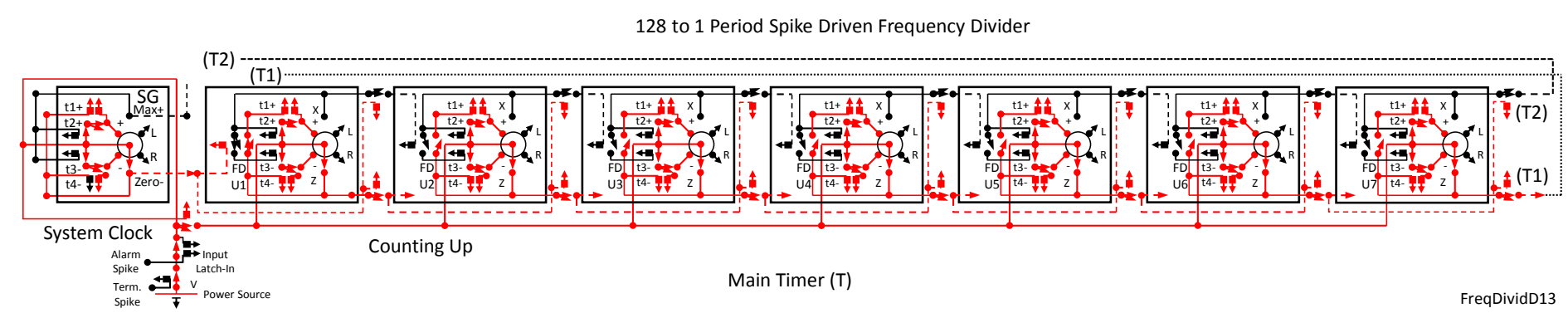

Figure 24 The 128 to 1 Frequency Divider can allow 128 positive muscle cells and another 128 negative muscle cells to be activated within a single pulse period using only seven (7) Frequency Divider Units (FDU's).

The time between the outputs of the Frequency Divider (T1) and (T2) forms the Period Pulse Train that acts as the carrier frequency in a system using pulse width modulation.

\subsubsection{Muscle motion using Pulse Width Modulation}

The frequency generator and frequency divider can be connected to a muscle by the Period Timing Bus consisting of conductors (T1) and (T2), as shown in Figure 25, below. The three-unit Frequency Divider can activate eight positive muscle cells and release eight negative muscle cells within each Standard Pulse Period, providing a good representation of muscle motion. 


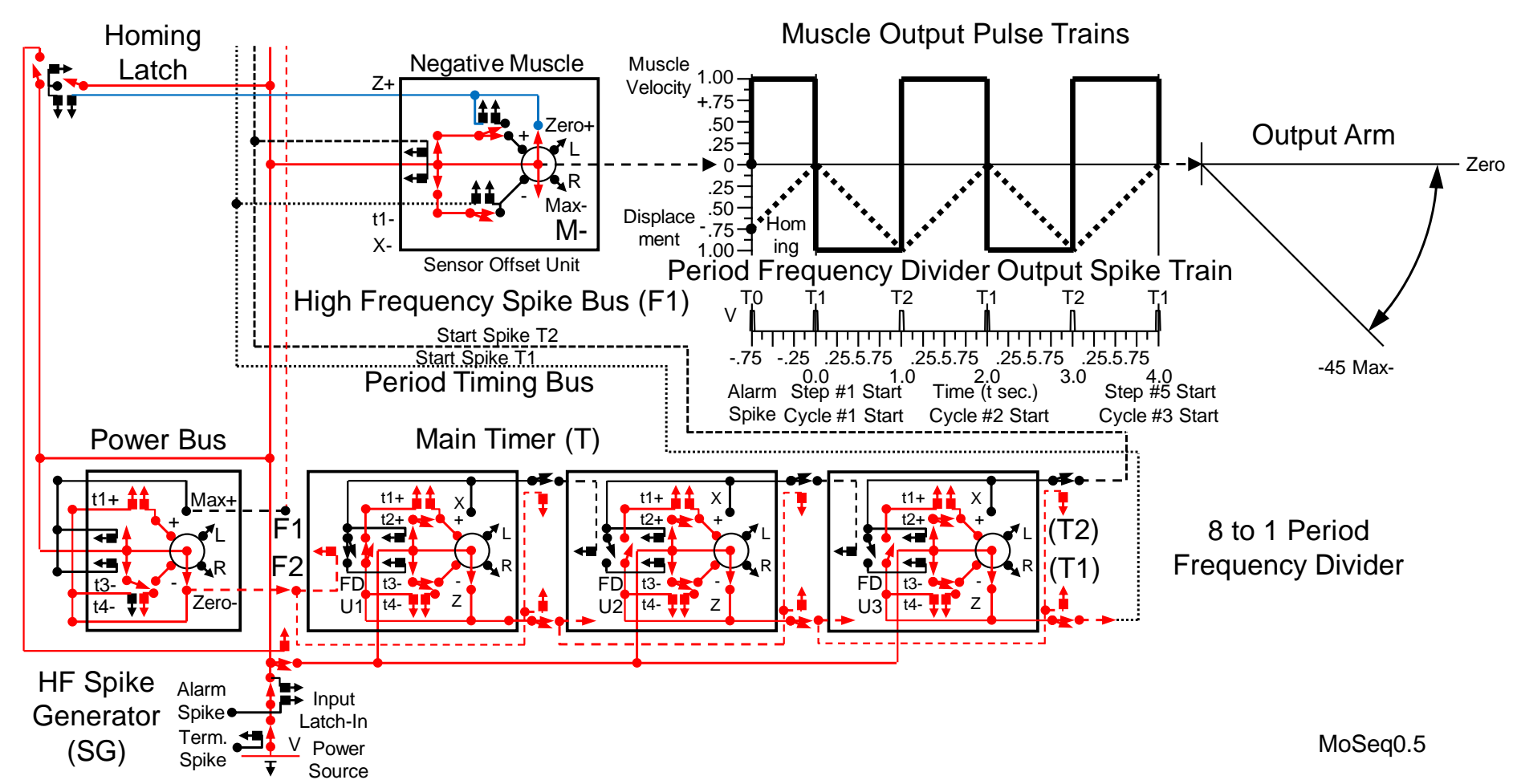

Figure 25 Using a Period Timing Bus (T1) and (T2), a muscle moves in pulses determined by the outside timing source.

The Negative Offset Muscle (M-) is started by a spike on the (T1) Terminal. This moves the Output Arm 5.6 degrees with each (F1) pulse from the Spike Generator, for a total of 45 degrees after the eight spikes from the HF Spike Generator in one Pulse Period when the Frequency Divider produces a spike at the (T2) Terminal, which stops the negative movement of the muscle at the end of the Standard Pulse Period and starts the reset motion. Since this Muscle Unit is started at fixed intervals, there is no information in the pulse train unless it is stopped by an Off Set Spike at some time within the Pulse Period. So the information in the pulse is contained in the timing of the Offset Spike, and the muscle is called a Sensor Offset Unit (SOFU).

(Until now, the rotation created by the muscle has been indicated by the rotation of the Rotor Arm within the logogram of the muscle unit, which has been $+/-180$ degrees. The Rotor Arm shown in logograms of the muscle units does not actually make contact with the Max and Zero Terminals. As shown previously, these contacts are made by the Max and Zero Muscle Cells within the Muscle Unit. So the Maximum Output Arm motion can be whatever is desired, and can be determined by the Rotor Diameter, the number of Muscle Cells, and the extent of movement of each Muscle Cell. In the example above in Figure 25, the maximum rotation of the Output Arm is shown to be 45 degrees. For the eight Muscle Cells with a 1/16 inch movement for each cell in the sixteen cell muscle shown previously, the circumference of the Rotor Arm needs to be one inch to produce the 180 degree rotation. So the Rotor circumference needs to be four inches to produce the 45 degree rotation of the Output Arm. This means the Rotor needs to be 1.27 inches in diameter. However, the Rotor Arm in the Logograms will continue to be shown to rotate $+/-180$ degrees so as to indicate the intrinsic $+1,0,-1$ amplitude values in symmetrical PWM.)

The Muscle Unit needs to home before it starts working, as shown previously. But the Main Timer (T) must not start until the Muscle Unit has homed to zero. Thus, the Muscle Unit needs to send a start signal spike to the Main Timer (T) when the Muscle Unit reaches zero, as shown by the Homing Latch in Figure 25.

If there is no Termination Spike, the Muscle Output Pulse Train repeats regularly. As such, it contains no information. It is similar to the television raster, which is a scan pattern that produces an all-white or all-black picture on the screen. So some time information needs to be introduced to the pulse train. 
Pulse width modulation

\subsubsection{Using a sensor to interrupt a motion pulse}

A Right Sensor (RK) can be added to the circuit with contacts that are closed when the Output Arm contacts an Object, as shown in Figure 26, below.

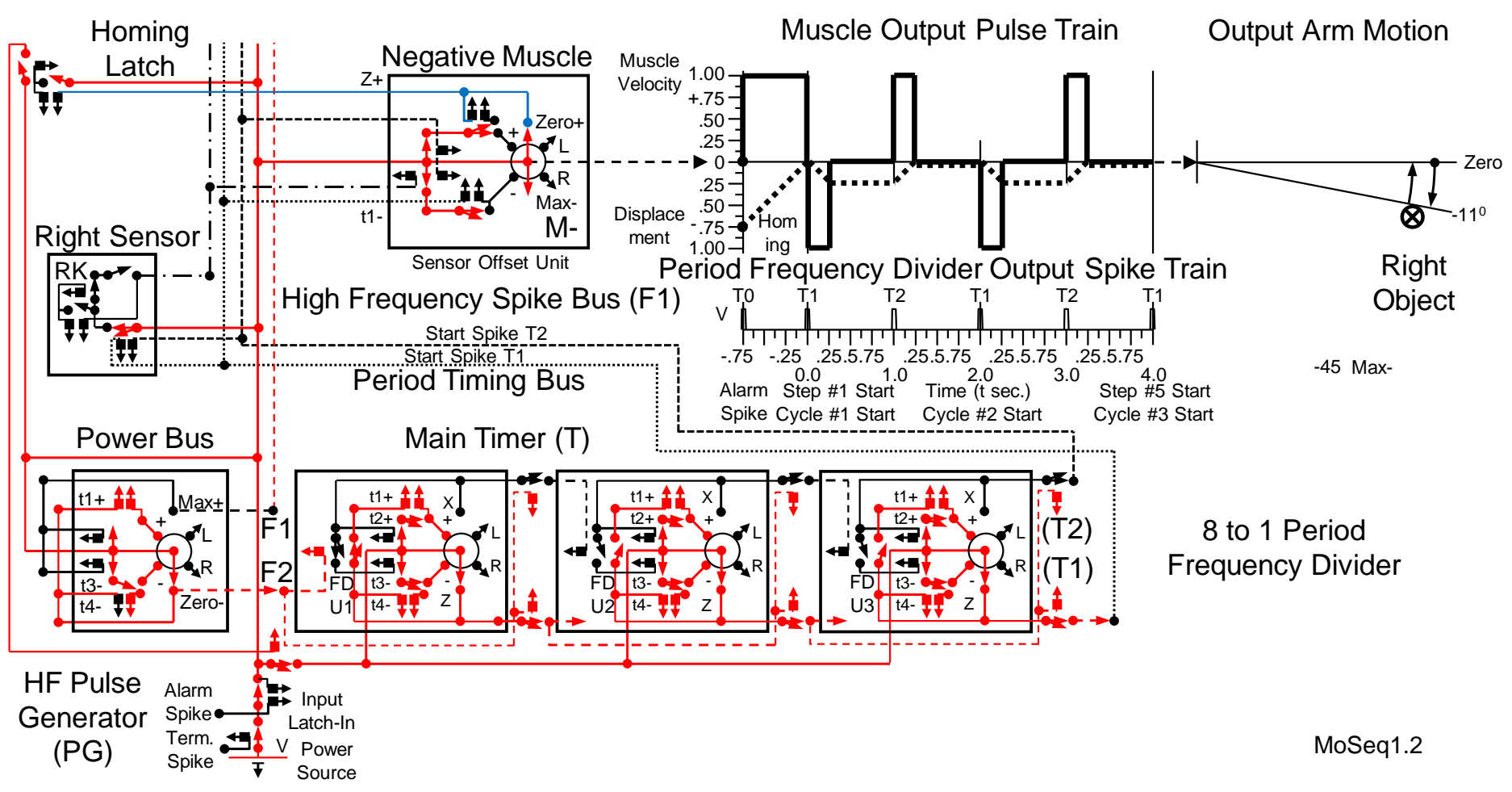

Figure 26 The Negative (Right) turning Offset Muscle produces a pulse train that is determined by the position of an object contacted by the Right Sensor (RK) on the Output Arm and the outputs (T1) and (T2) of the Main Timer (T).

The Right Sensor (RK) output stops (offsets) the pulse from the (F1) spike train to the Max (-) muscle cell, and the spike on the conductor (T2) causes the muscle to reset to Zero (+). Then a spike on the (T1) conductor causes the muscle to move toward Max (-) again.

The Output Arm Motion Pulse Train contains information as to the location of an object. Notice that the Negative Muscle (M-) temporarily stores the time of contact with the object until the next period, when it duplicates this time of contact when it finishes resetting to zero.

\subsubsection{Using an Offset and an Onset muscle together}

An Onset Muscle is turned "on" by an Onset Spike from the Right Sensor (RK), in this case, it occurs sometime in the Standard Period, as shown by the (M+) Onset Muscle in Figure 27. Since the Onset Muscle is not started by the Main Timing Bus, the information is contained in the timing of the onset spike from the Right Sensor (RK). So it is called a Sensor Onset Unit (SOSU), as shown Figure 27, below. 


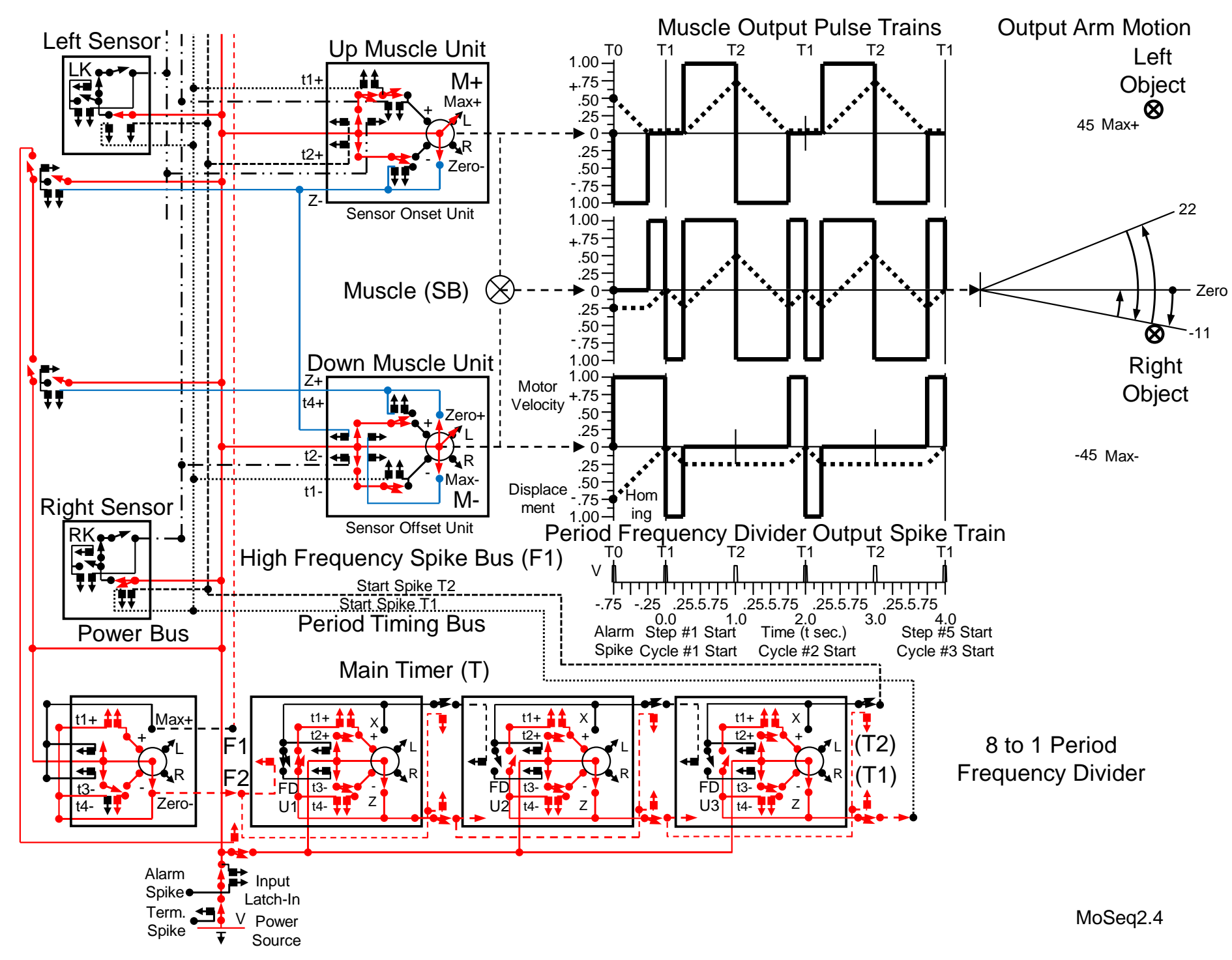

Figure 27 The antagonistic Muscle Units (M-) plus (M+) form a single Muscle (SB) that moves its Output Arm toward and away from an object, and back to its Zero (home) position within a two-period cycle.

In the Bottom Muscle (SB), the Offset Unit (M-) is started by T1, and the Onset Unit (M+) is enabled by (T1) and is started by a spike from the Right Sensor (RK), and is reset by a spike from (T2). The Offset Muscle (M-) is reset by a spike from the Zero Terminal (Z-) Of the Onset Unit (M+). The information in the Negative (Offset) Muscle (M-) is started by the Start Spike (T1) from the Main Timer and the Offset Spike from the Right Sensor (RK), and the information in the Positive (Onset) Muscle (M+) is determined by the time of the Onset Spike from the Right Sensor (RK) and the End Spike (T2) from the Main Timer.

(The position of the Contact Arm in the logogram of the Muscle is like a fuel gauge in an automobile. It does not show how much fuel is in the tank, quantitatively. But does show qualitatively how full the tank is from a range of 0 "empty" to 1 "full" with some points in between like $1 / 4,1 / 2$, and $3 / 4$ full. It is very useful because it shows the state (width) of the muscle pulse within the Standard Pulse Period.)

\subsubsection{A Bottom Muscle Unit responding to objects on both sides of its Output Arm}

The Offset and the Onset Muscles can respond to objects contacted by the Right and Left Sensors as shown in Figure 28, below. 


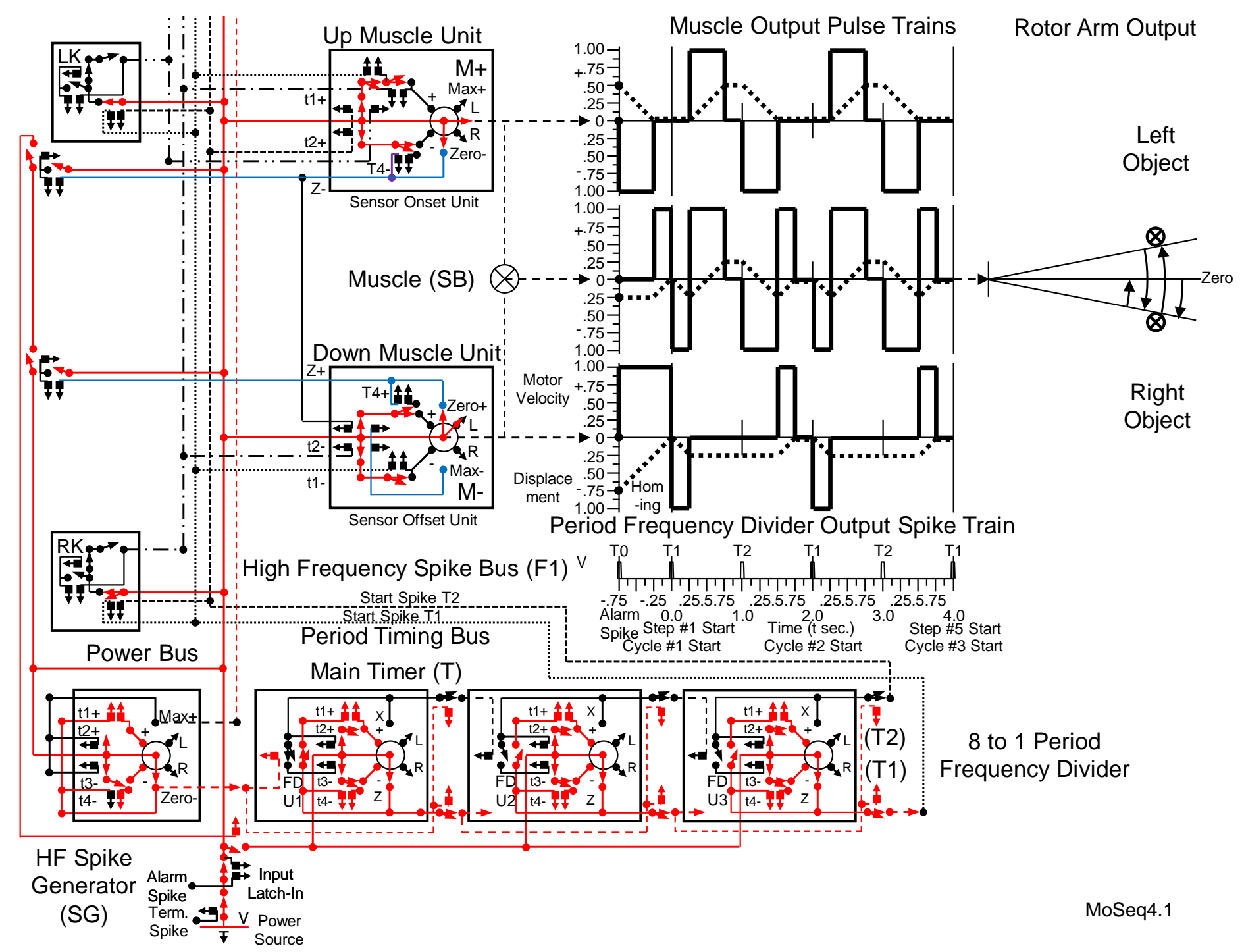

Figure 28 In this Bottom Matrix (SB) the Offset Muscle (M-) is started first and is stopped by the Right Sensor (RK). It is reset by the Zero (Z-) output of the Onset Muscle (M+). The Onset Unit (M+) is started by the Right Sensor (RK), stopped by the Left Sensor (LK), and is reset by $\mathrm{T} 2$.

The Offset Muscle (M-) and an Onset Muscle (M+) can be connected to the Timing Unit and Timing Bus forming the symmetrical Bottom Matrix (SB) shown in Figure 28, above. The Positive (Left) turning Sensor Onset Muscle (M+) is enabled by the T1 Period Timing Bus, and is started by a spike from the Right Sensor (RK). It is stopped and reset by the T2 bus.

The location of the two objects is encoded in the pulse train created by the Muscle (SB). There will be a unique pulse train created for every different location of the objects. The number of uniquely different pulse trains (variety) is the measure of information (the improbability of a given pulse train given the number of possible pulse trains) (Brown R. A., Machines that learn, 1994).

This Bottom Muscle (SB) can function successfully by itself. But the system will work better when it is connected to a Top Muscle (ST), as will be shown in Figure 29, below.

\subsubsection{The Inverted Top Muscle}

The Bottom Muscle (SB) can be duplicated and inverted forming the top Muscle (ST) as shown in Figure 29, below. 
Pulse width modulation

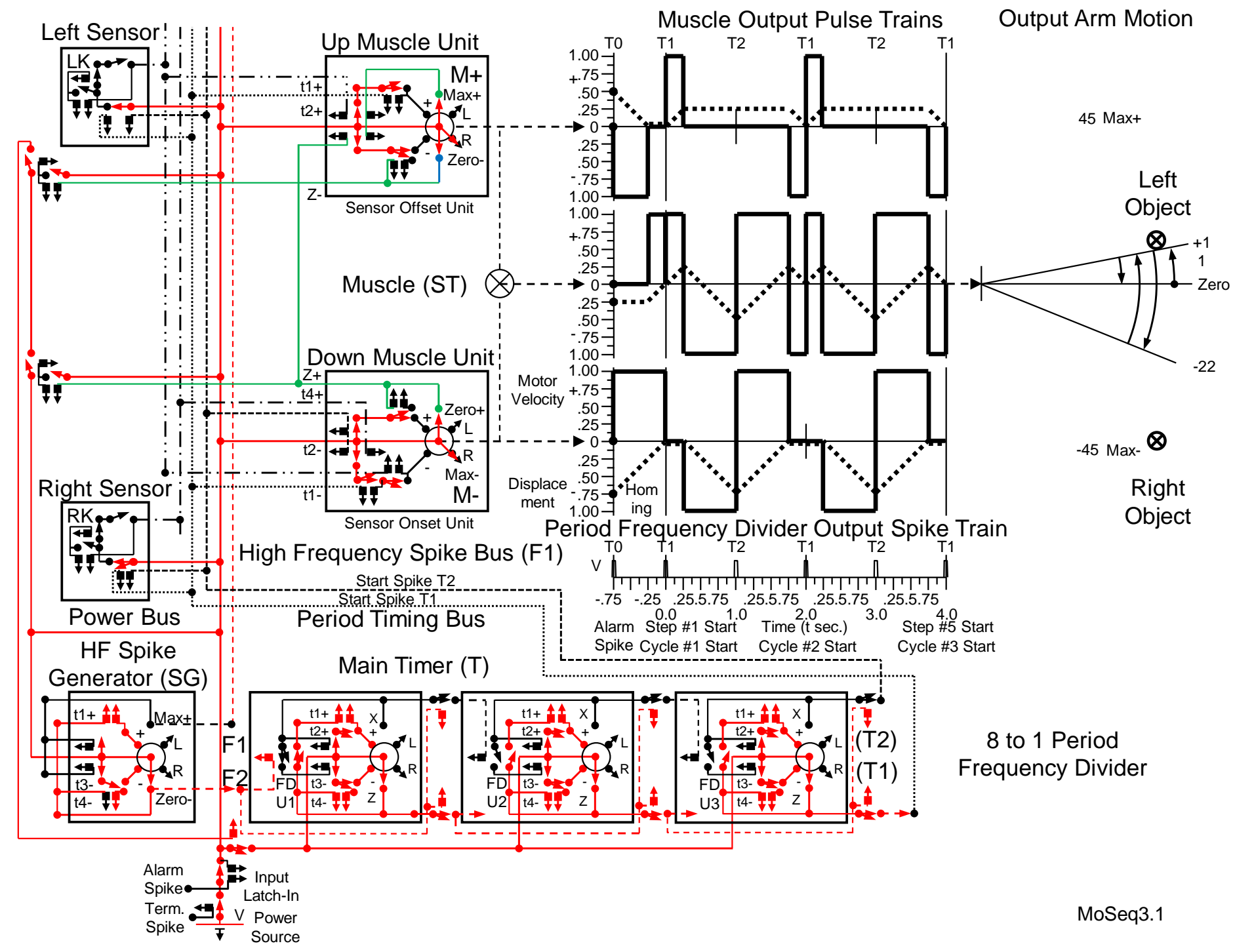

Figure 29 The Top Muscle (ST) responds to an object contacted by the Left Sensor (LK).

The Left Sensor stops the Offset Up Muscle Unit and starts the Onset Down Muscle Unit when it contacts an object.

\subsubsection{A Top Muscle Unit (ST) responding to an object on its left side}

The Right Object may be in the path of the Output Arm also, as shown in Figure 30, below. 


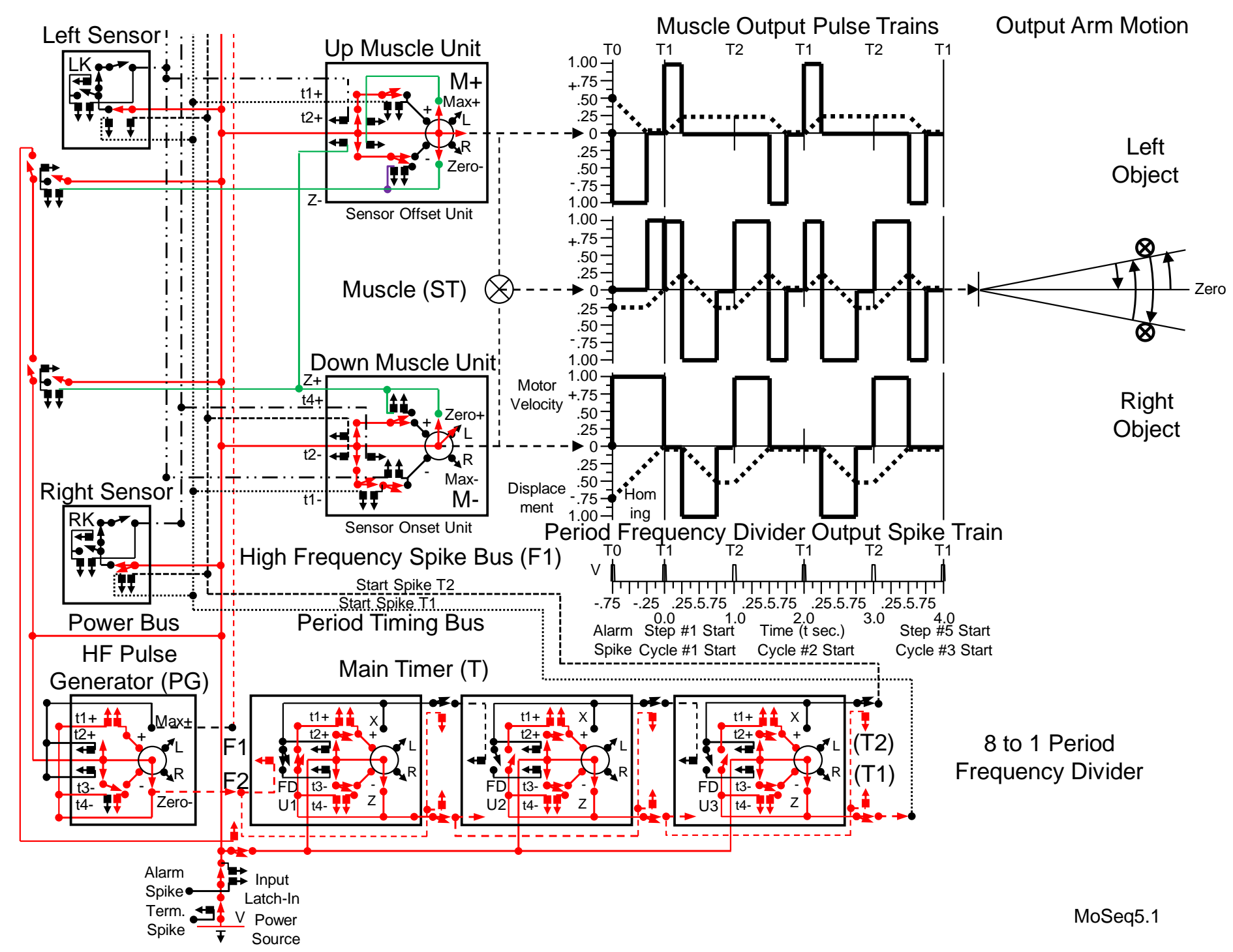

Figure 30 The Right Sensor (RK) stops the Down (Onset) Muscle Unit (M-), and the Left Sensor (LK) stops the Up (Offset) Muscle Unit (M+) when the sensors contact an object.

The Down Muscle Unit (M-) resets the Up Muscle Unit (M+) when the Down Muscle Unit gets to Zero, creating the pulse train shown above from the Muscle (ST). The Output Arm pulse train contains the information pertaining to the location of two objects relative to the location of the muscle of an animal.

The antagonistic pairs can be duplicated and inverted as shown in Figure 31, below. This extends the range of motion of the Muscle Output Arm.

\subsection{Object avoidance in a Top/Bottom symmetrical matrix muscle group}

Putting the Bottom and Top Muscle Units together forms a Symmetrical Muscle Group that is the foundation of Symmetrical, Zero-Sum Pulse Width Modulation (s z PWM). This architecture provides the potential for order in systems that are highly complex.

\subsubsection{A Symmetrical Muscle group with no objects in the path of its Output Arm}

The Top (ST) and Bottom (SB) muscles shown above can put together into one Symmetrical Muscle that produces a wider range of motion than is available in a single Top or Bottom Muscle, as shown below in Figure 31. 


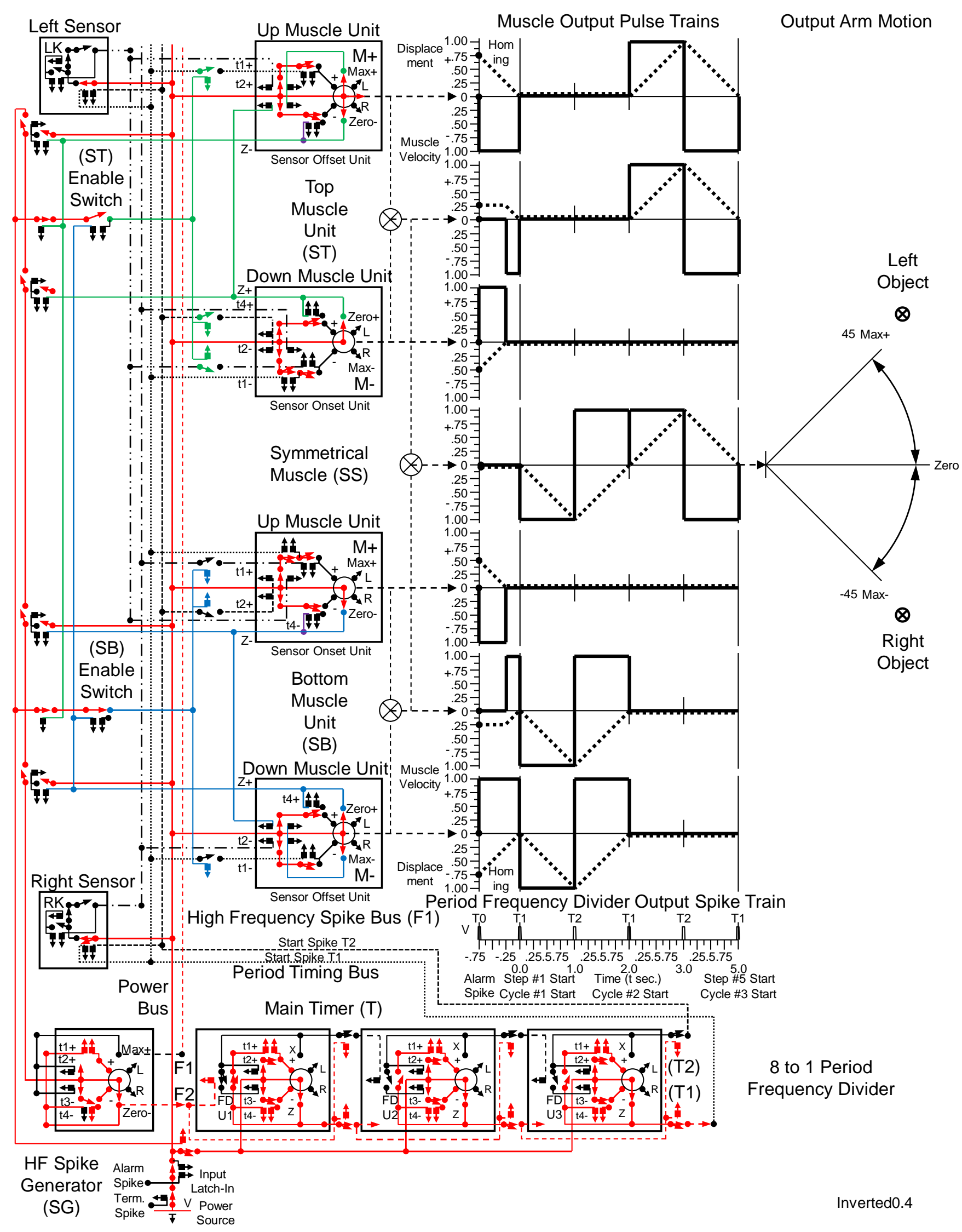

Figure 31 The Symmetrical Muscle (SS) consisting of an inverted pair of Muscle Units (SB) and (ST) that are enabled (activated) alternately.

The Left Sensor and Right Sensor are connected to the Top Muscle Unit (ST) and the Bottom Muscle Unit (SB). This allows the Symmetrical Muscle (SS) to respond to an object on the left and right side of the Output arm, as shown below in Figure 32. The ST Enable Switch and the SB Enable Switch enable the Bottom Muscle Unit (SB) to be expressed first, and the Top Muscle Unit (ST) to be expressed when the Offset Unit (M-) in the Bottom Muscle Unit (SB) completes its motion at its zero position. The ST Enable Switch and the SB Enable Switch also allow the Top Muscle Unit (ST) to enable the Bottom Muscle Unit (SB) when the Offset Unit (M+) in the Top Muscle Unit (ST) completes its motion at its zero position.

\subsubsection{A symmetrical muscle group with an object on the right side of its Output Arm}


An object is shown to be on the right side of the Output Arm in Figure 32, below.

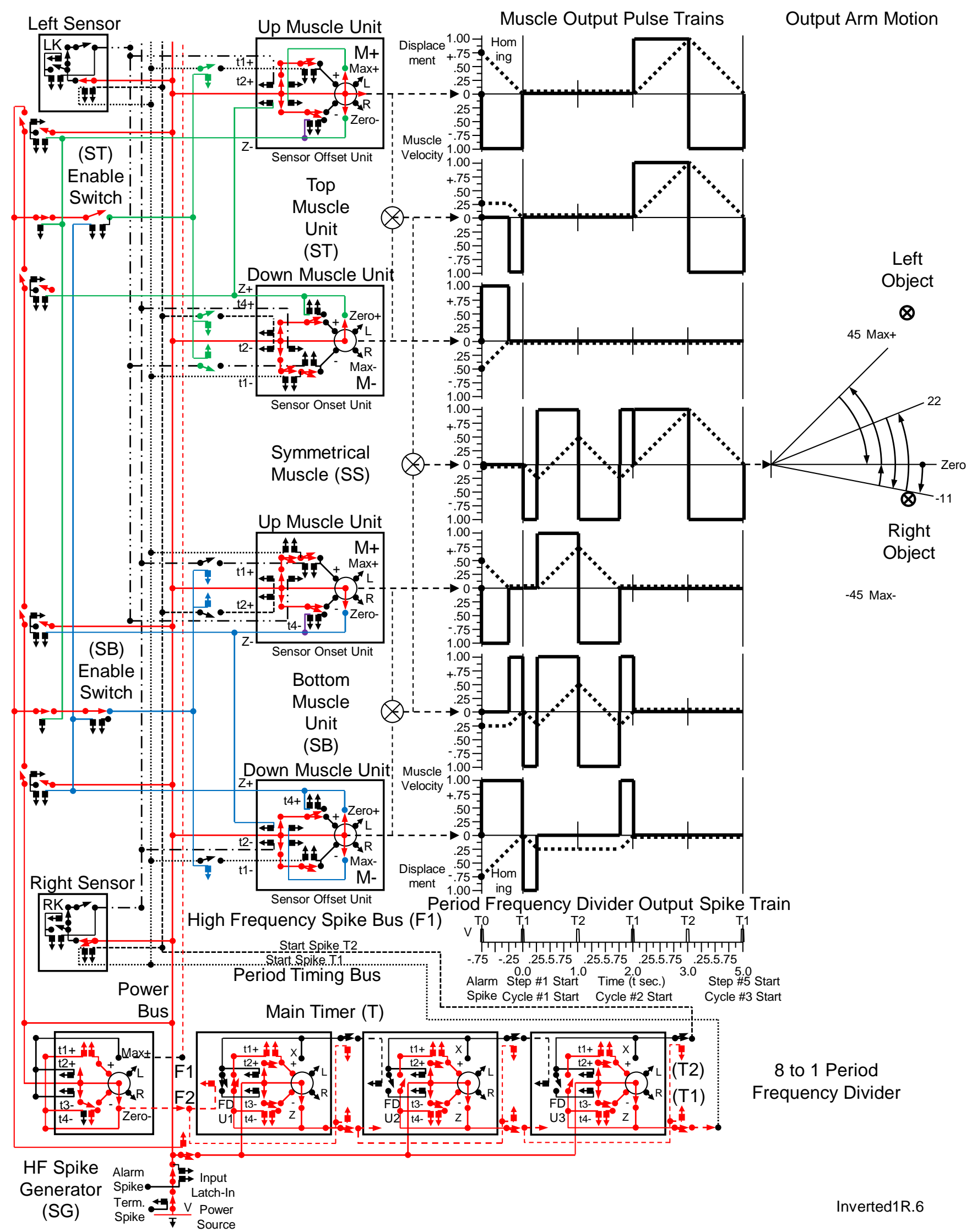

Figure 32 In a Symmetrical Muscle (SS), the Top Muscle (ST) extends its range of motion when the object is just on the right side of the Output Arm.

This symmetrical configuration of The Top Muscle (ST) and Bottom Muscle (SB) is the foundation of the muscles and neural circuits shown in this paper.

\subsubsection{A symmetrical muscle group with an object on the left side of its output arm}

The object may be placed on the left side of the Output Arm, as shown below in Figure 33. 


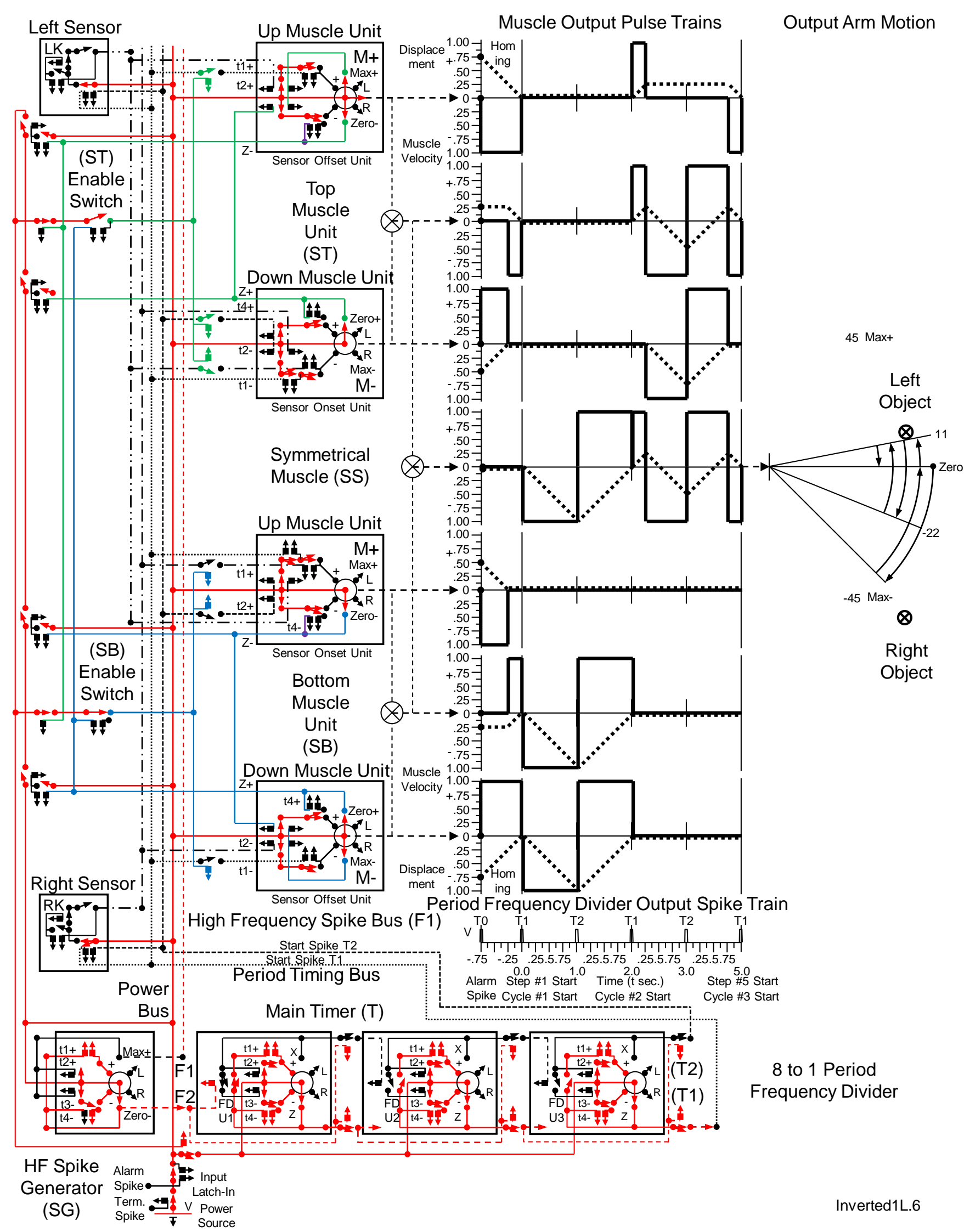

Figure 33 In a Symmetrical Muscle (SS), the left side of the muscle (Matrix ST) responds to the object on the left side of the Output Arm after the right side of the muscle (Matrix SB) completes its full range of motion.

The Enable Switches allow just one muscle unit to operate at a given time.

\subsubsection{A symmetrical muscle group with objects on both side of its output arm}

The object may appear on both sides of the Output Arm, as shown in Figure 34, below. 


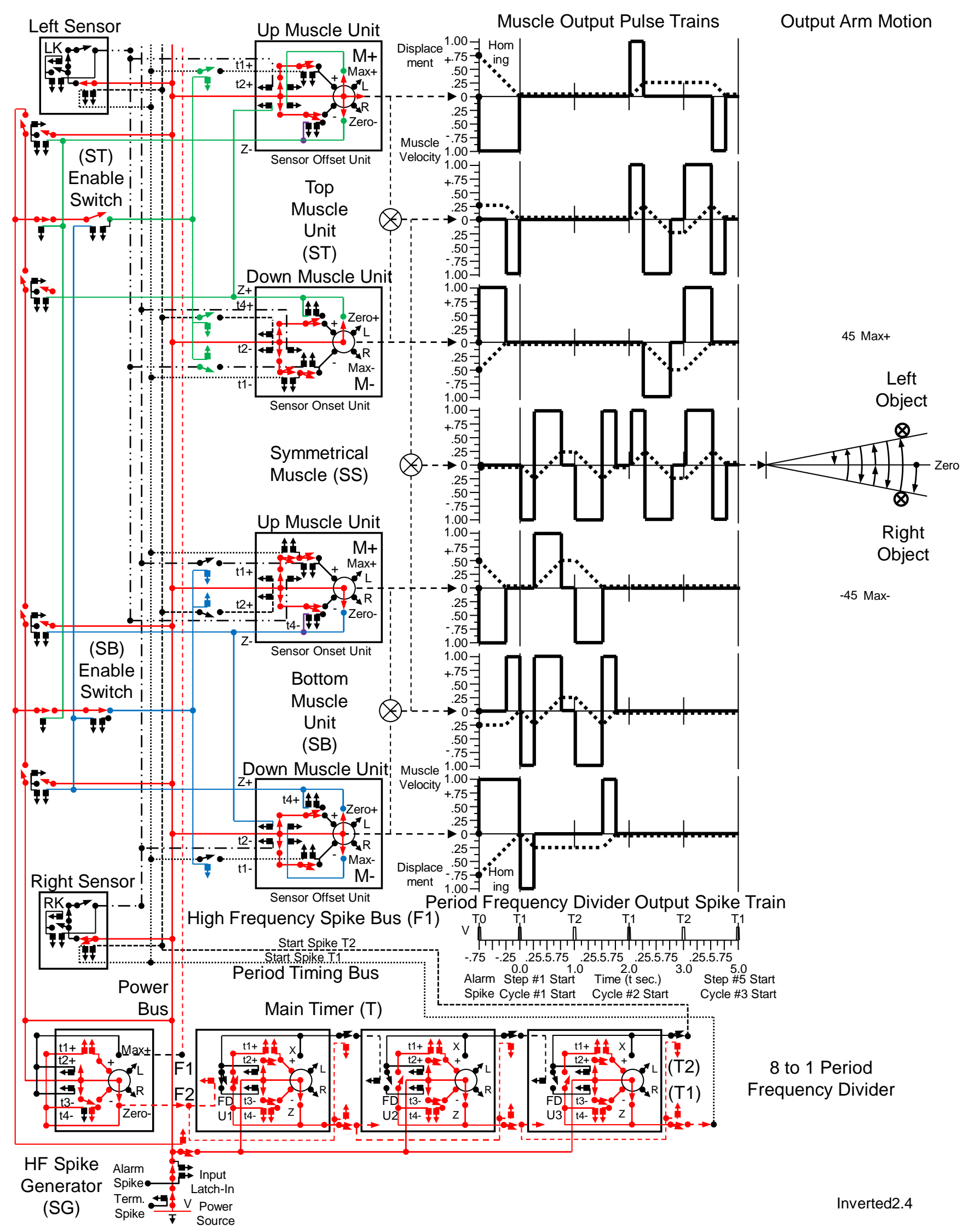

Figure 34 In a Symmetrical Muscle (SS), when an object is present on both sides of the Output Arm, the muscle is able to produce the maximum amount of motion allowed by the two objects.

The pulse train created by the Symmetrical Muscle (SS) contains the information as to the location of objects relative to the position and orientation of the animal.

\subsubsection{A symmetrical muscle group with objects at different positions on both sides of its output arm}

An object can be any position on either side of the Output arm. So the Output arm will move back and forth between the two objects while returning to Zero at the end of each Cycle, as shown in Figure 35, below. 


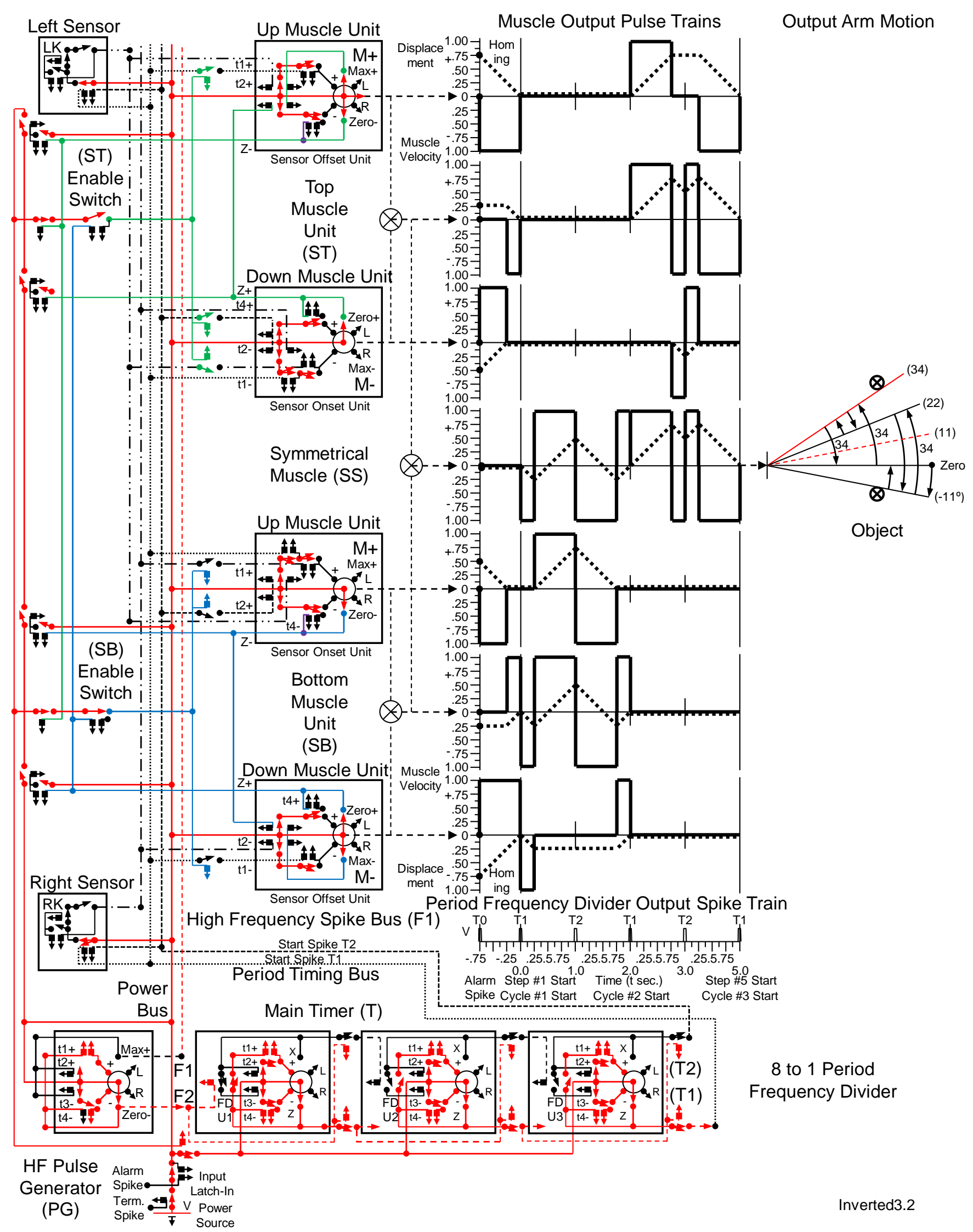

Figure 35 The Symmetrical Muscle (SS) creates a symmetrical motion sequence between the two objects, which is centered in a position midway between the two objects.

Up to this point, muscle units have been used to create motion and store information, temporarily, so that the sum of their motion equals zero after a motion cycle according to the equation: Positive Pulses $+($ Negative Pulses $)=$ zero. This zero-sum process keeps the individual muscle homed at Zero, which keeps the system from getting lost, even though the midpoint of the output motion between the two objects has shifted off Zero. This is equivalent to keeping bank accounts balanced and reconciled, which is the foundation of financial order, while the total assets and liabilities may change with changes in loans, deposits, and withdrawals.

\subsection{Permanent memory using an auxiliary muscle unit}

An animal, like a crinoid, may benefit by remembering the location of an object in its environment. Rather than contacting 
Pulse width modulation

the object in its path a second time, the animal may avoid contact with the object by remembering its location, and creating a response that turns the animal away from the object before contact is made. Rather than repeatedly bumping into an object with its sensor, the time based system using (PWM) can adjust its zero position so as to avoid an object and maintain the full motion potential of its contact arm. This is done by adding an auxiliary muscle in both bottom and top muscle units that permanently stores the time location of the object.

4.6.1 With no objects in the path of the Output Arm of the Symmetrical Muscle Group with Auxiliary Muscle units With no objects in the path of the Output Arm, the Output Arm can move to its full extent in both directions, as shown below in Figure 36, below. 
Pulse width modulation

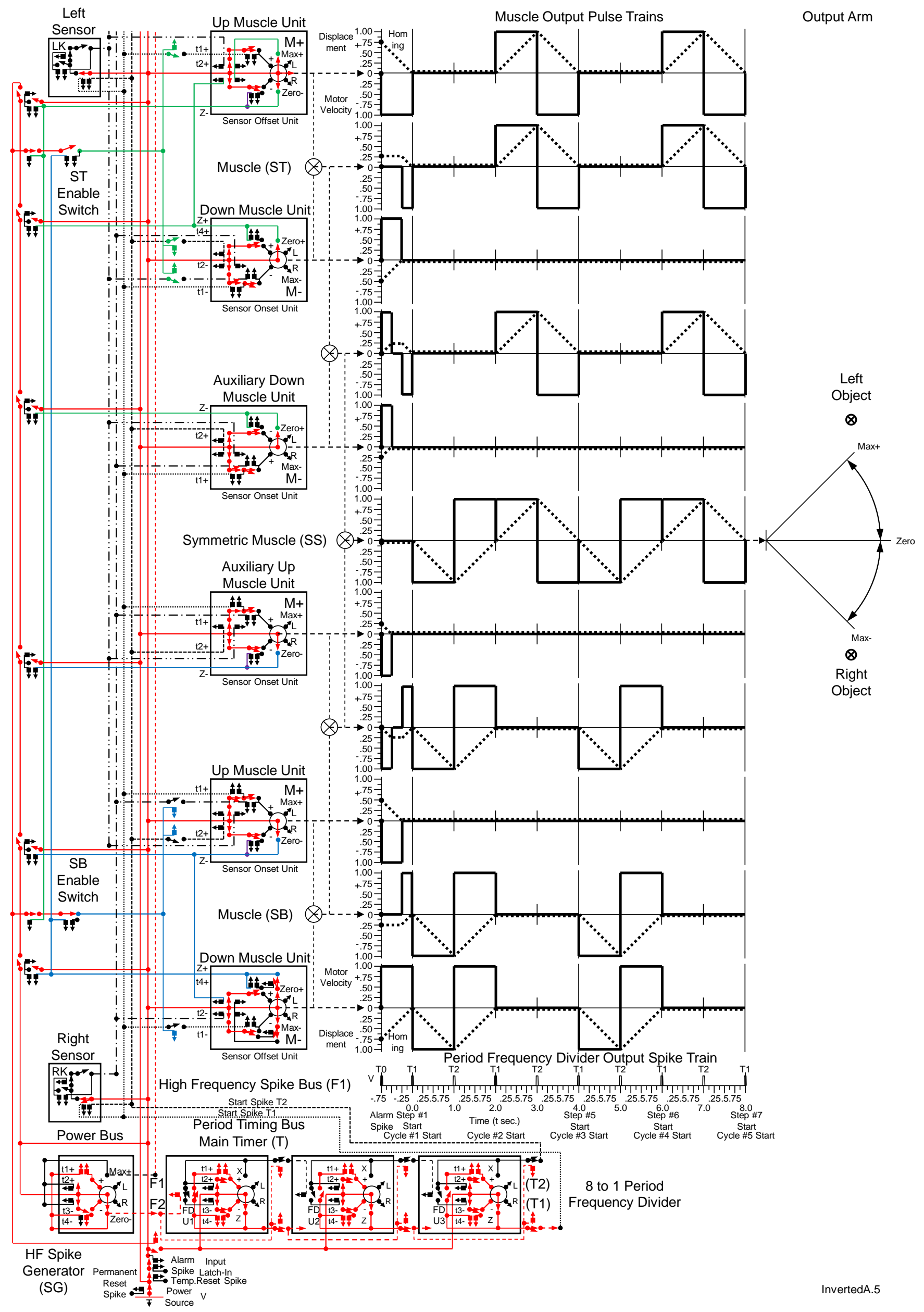

Figure 36 An Auxiliary Up-Muscle and Auxiliary Down-Muscle are activated only if an object is present in the path of the Output Arm.

With no objects present, the Output Arm moves as a scanner through its full range of motion, as shown before in Figure 31

\subsubsection{With an object on the right side of the Output arm}

When an object is contacted, an Auxiliary Muscle is onset and moves until stopped at the end of period. It remains in that position indefinitely without being reset, as shown in Figure 37, below. 
Pulse width modulation

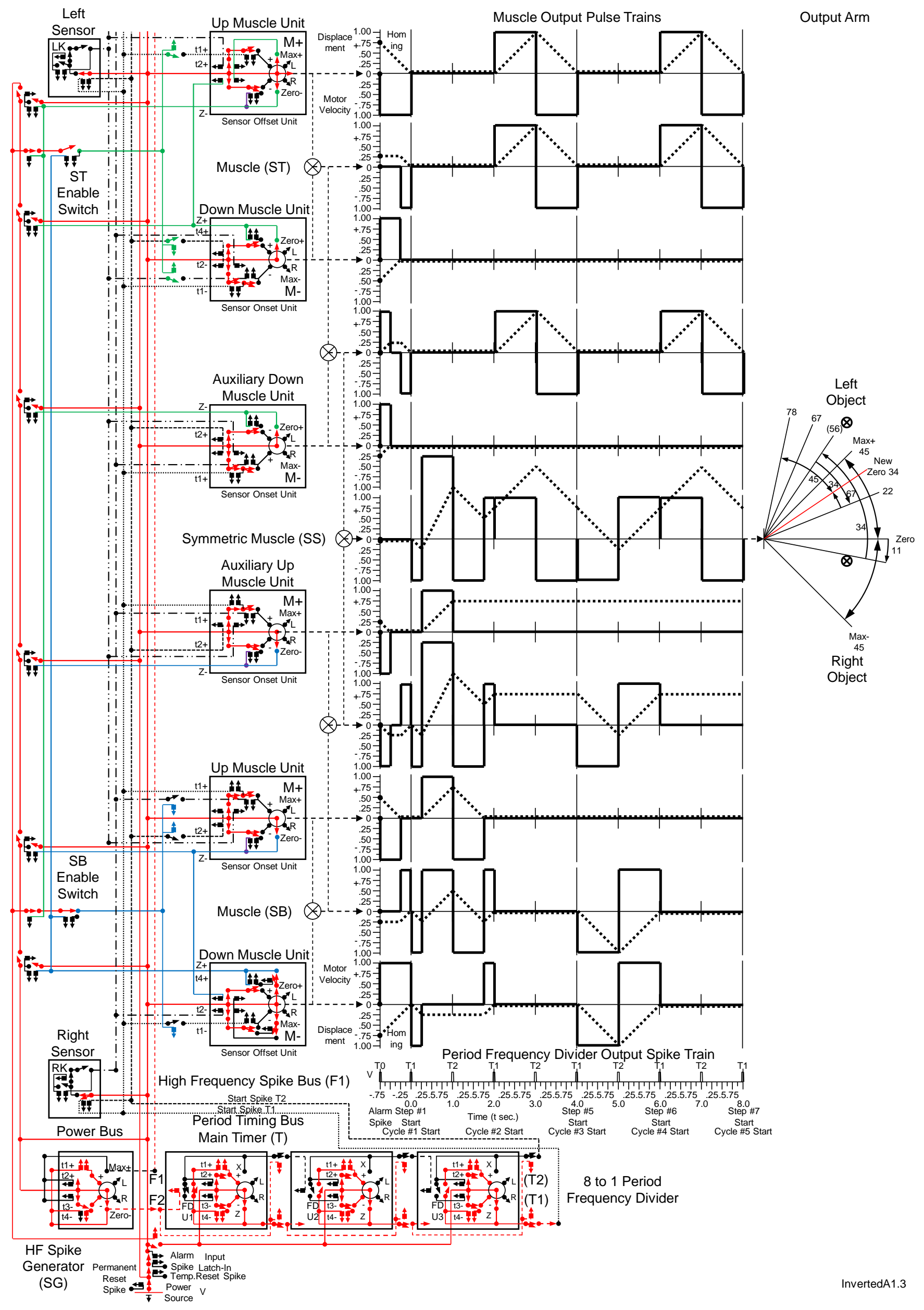

Figure 37 When an object is contacted by the Right Sensor, an Auxiliary Up Muscle Unit in the bottom Muscle Unit (SB) is set permanently to the time of contact within the first period. The motion of the Auxiliary Muscle Unit is added to the motion of the Symmetrical Muscle (SS), creating a new output trajectory.

Notice the all of the muscles except for the active Auxiliary Muscle Unit home to Zero at the end of each cycle. However, the Output Arm is in a new (non-zero) position at the end of has a new of each Cycle. This feature would allow an animal to move around the midpoint of the maximum extents of it movement.

4.6.3 With an object on the left side of the Output Arm 
When an object is located on the left side of the Output Arm it moves as shown in Figure 38, below.

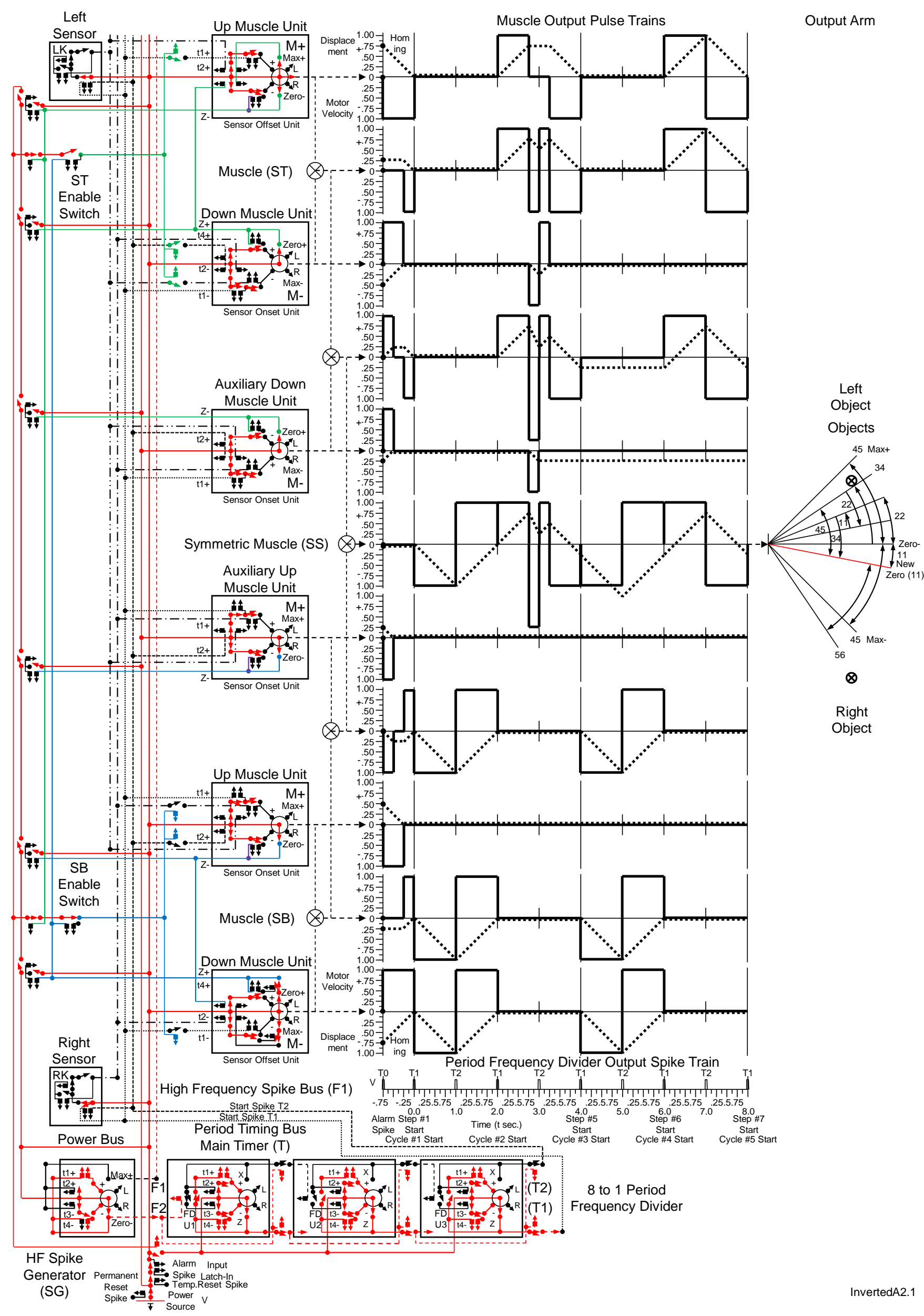

Figure 38 When an object is contacted by the Left Sensor, an Auxiliary Down Muscle in the top Muscle Unit (ST) is permanently set to the time of contact near the end of the third Period.

The maximum range of motion can now be made by the Output Arm without contact with the object.

\subsubsection{With an object on both sides of the Output Arm}

The objects may be located on both sides of the Output Arm, as shown in Figure 39, below. 
Pulse width modulation

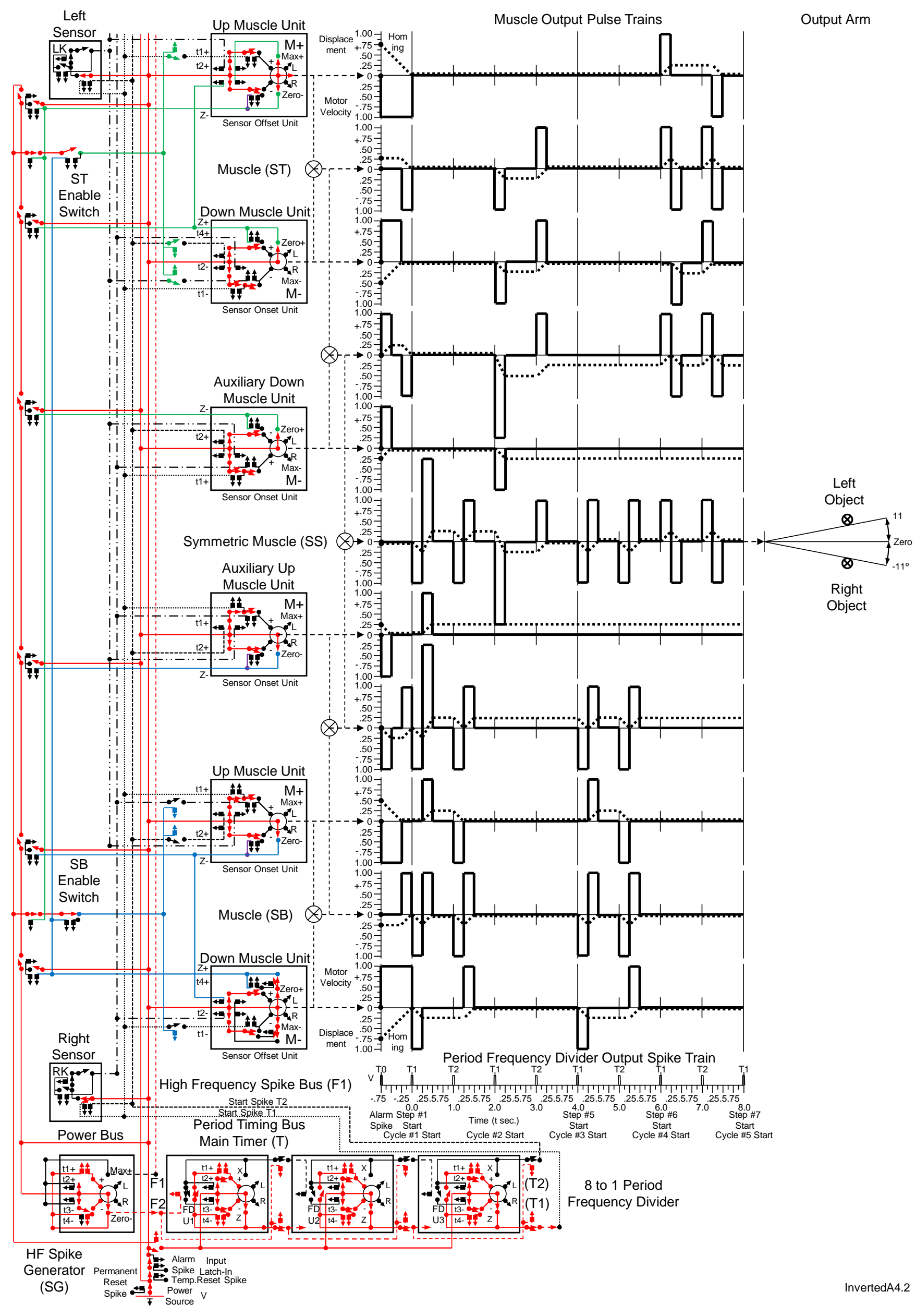

Figure 39 When objects are contact by the Right Sensor and Left Sensor, the time of contact is recorded permanently by the Auxiliary Muscles in both matrices, and the Symmetrical Muscle (SS) moves sequentially around a point midway between the two objects.

The output pulse pattern is determined by the location of objects in the environment.

\subsubsection{With objects at different locations on both sides of the Output Arm}

The objects do not have to be equally spaced around the Home or Zero location, as shown in Figure 40, below. 
Pulse width modulation

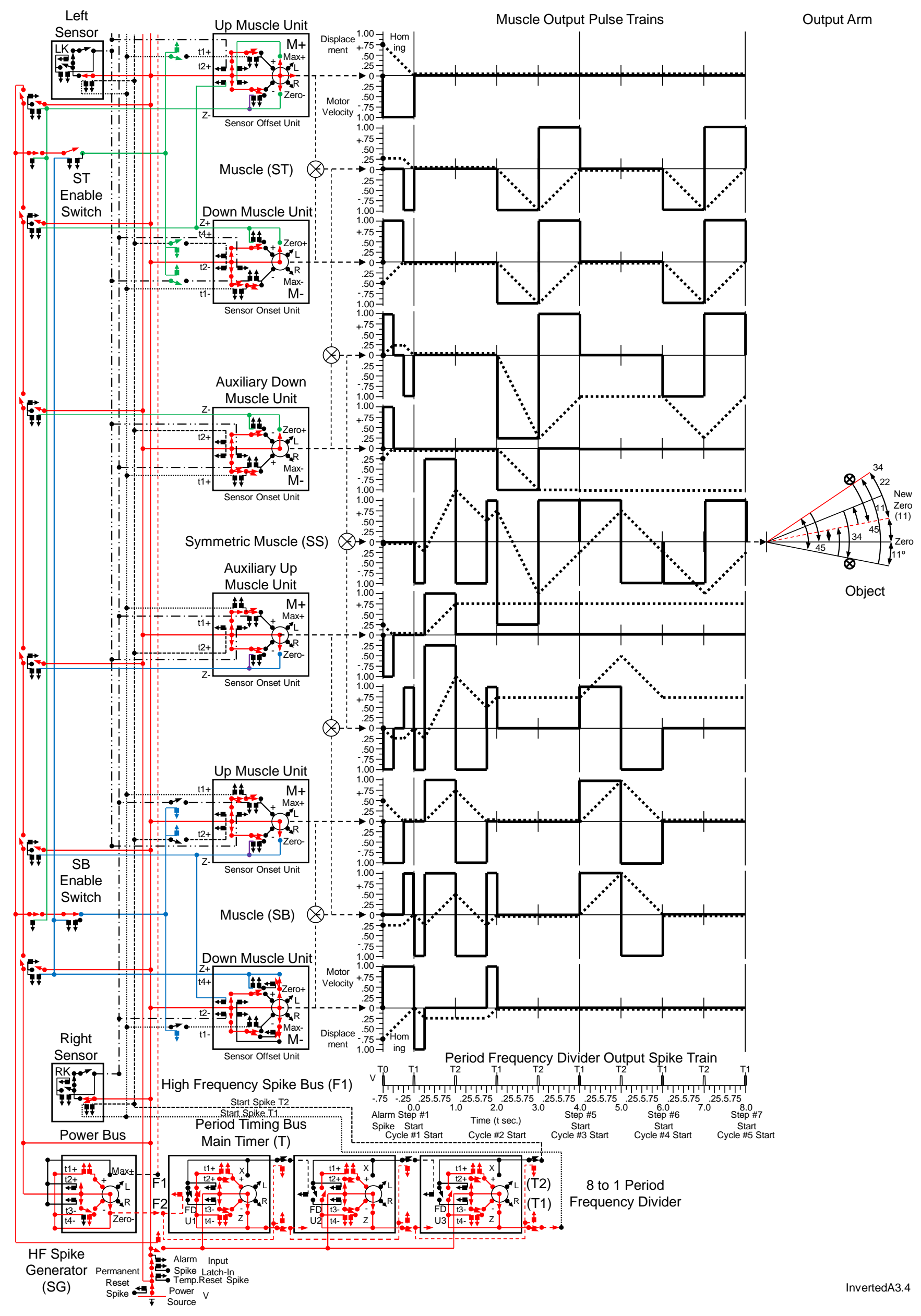

Figure 40 The average position of the Output Arm is located midway between the two objects, which creates a new external zero when the objects are located at different distances from the original zero.

The permanent memory allows the animal to move through the greatest motion between fixed objects without being injured by the contact objects. This is beneficial for an animal that lives in a burrow since it can travel through the burrow without making contact with the walls of the burrow. The new zero position represent a memory of a new body position that can be used for navigational purposes. It represents the angle of the burrow relative to direction of the animal before entering the burrow. 


\section{Pulse width modulation}

Note that all of the muscles except for the active Auxiliary (memory) Muscles are homed to zero at the end of each active cycle. The permanent memory units are the only non-zero pulse values in the muscle. So looking at the overall state of the muscle system, Positive Pulses + (Negative Pulses) = Permanent Memory Pulses.

The permanent auxiliary muscles are shown connected to a second power bus so the other muscles can be rehomed by a Termination Spike and an Alarm Spike. The permanent auxiliary muscles can be reset by the Permanent Reset Spike, only.

\subsection{Permanent Memory Co-cell Unit using a frequency divider}

Rather than using a muscle to remember the location of an object, it is possible to use a Memory Co-Cell made up of a frequency divider to record the time of contact within a Standard Pulse Period, and use that time information to cause an avoidance response at the same time in the next active period.

\subsubsection{Permanent memory using a Fixed Frequency Divider Co-Cell}

A frequency divider with one more Frequency Divider Units (FDU's) than the Main Timer can be started from a spike from the Object Sensor, and reset itself after four Standard Periods so that it reaches zero at the original time of contact in the fourth period. A frequency divider provides a much more efficient method of storing and duplicating this time of contact than using a muscle to produce the time delays equal to four Standard Pulse Periods.

The Frequency Divider, shown below, can store many more pulse times with far fewer contact elements (neurons) than a muscle. This Frequency Divider with 4 Frequency Divider Units (FDU's) can be used to replace four of the sixteen-step muscles shown in Figure 15, for example, as shown in Figure 41.

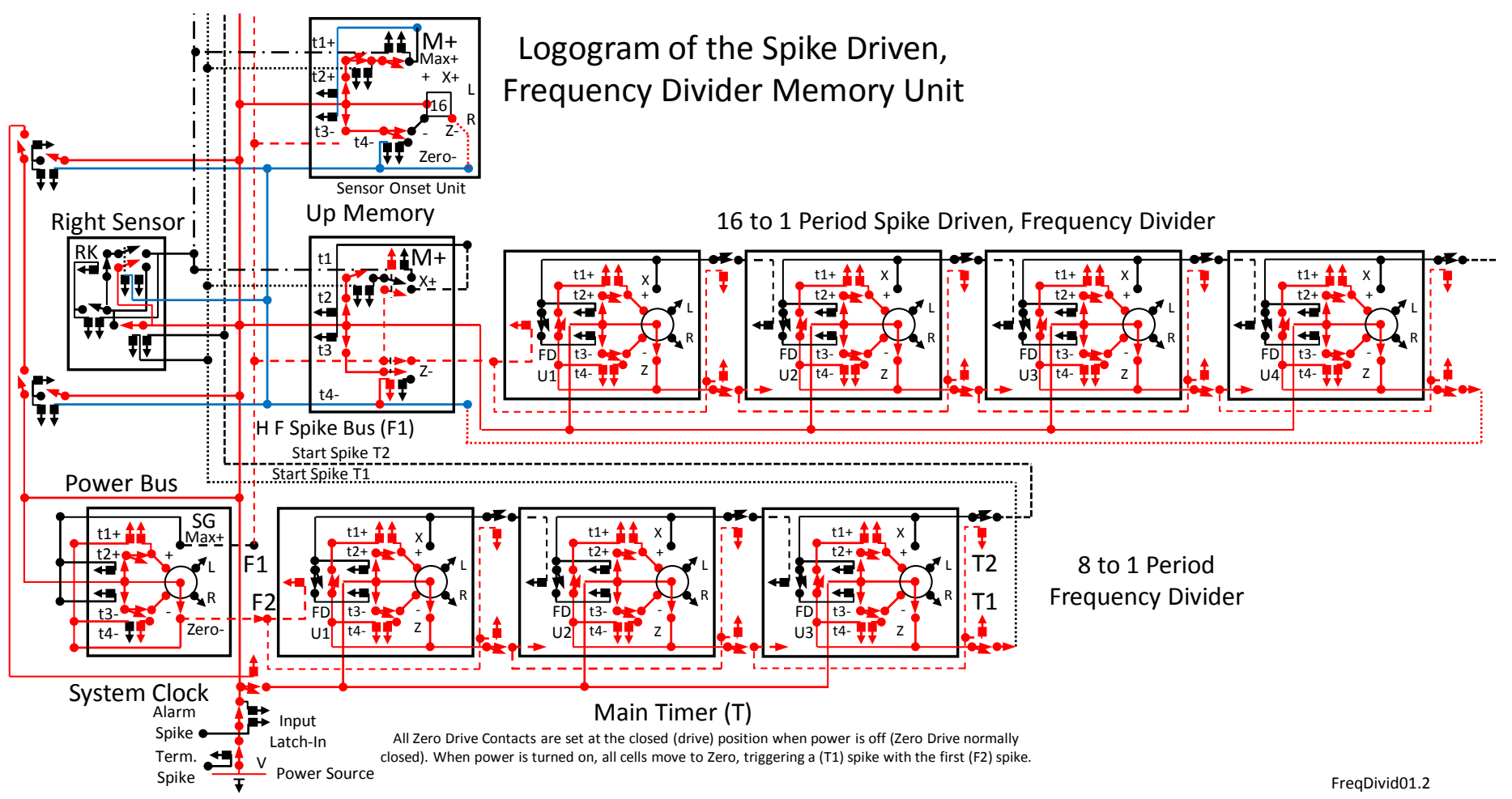

Figure 41 The 16 to1 Frequency Divider can be used as a fixed time Co-Cell in place of a muscle, as shown in the following Permanent Memory Muscle Control System.

The three-place, 8 to 1, Frequency Divider determines the time between a T1 spike and a T2 spike, which is the Standard Pulse Period. So the time between a T1 spike and the next T1 spike is two Standard Pulse Periods. Adding another Frequency Divider Unit (FDU) to the three-place Frequency Divider allows the four-place Frequency Divider to become a 16 to 1 Frequency Divider that produces a spike on its Zero Terminal after every four Standard Pulse Periods. 
Pulse width modulation

4.7.2 Bottom and Top Symmetrical Permanent Memory Muscle using frequency dividers as memory devices

The auxiliary muscles in Figure 36 can be replaced with a Frequency Divider Memory Co-cell Unit for each muscle that produces a memory that is held for four (4) Standard Pulse Periods. This is achieved by adding just one more Frequency Divider Units (FDU's) to the Frequency Divider Memory Co-Cell Unit shown in Figure 41, above, as shown in Figure 42, below.

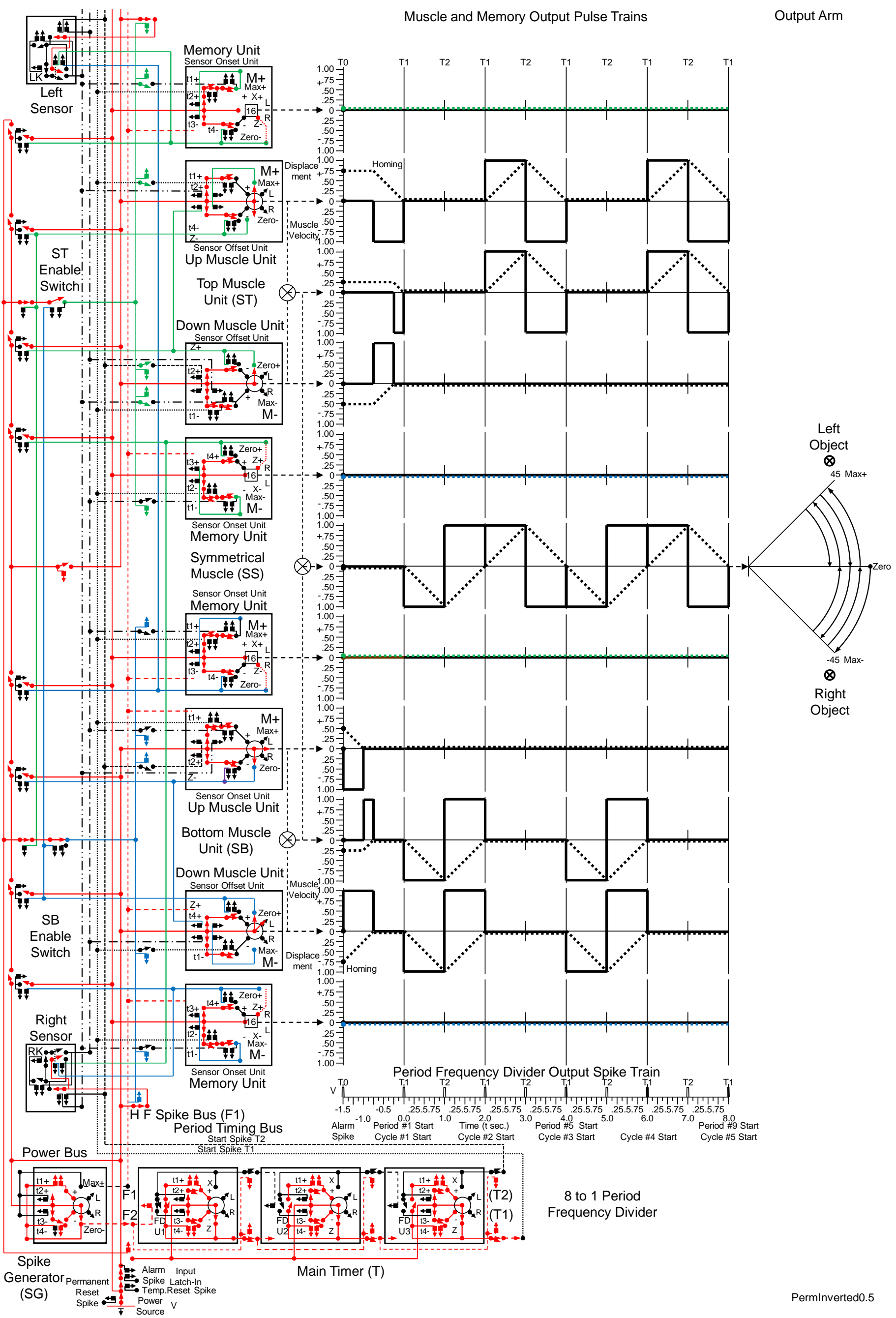


Pulse width modulation

Figure 42 When there are no objects in the path of the Muscle Output Arm of the Symmetrical Muscle (SS), the primary muscle units act as before as a scanner as shown previously in Figure 31.

The Output Arm motion may be viewed as a scanning process that contains no information.

4.7.3 A Symmetrical Muscle group that contacts an object on the right side of its Output Arm

When there is an object on the right-hand side of the Output Arm, the lowest Memory Register in the Bottom Muscle Unit (SB) is energized, as shown in Figure 43, below. 


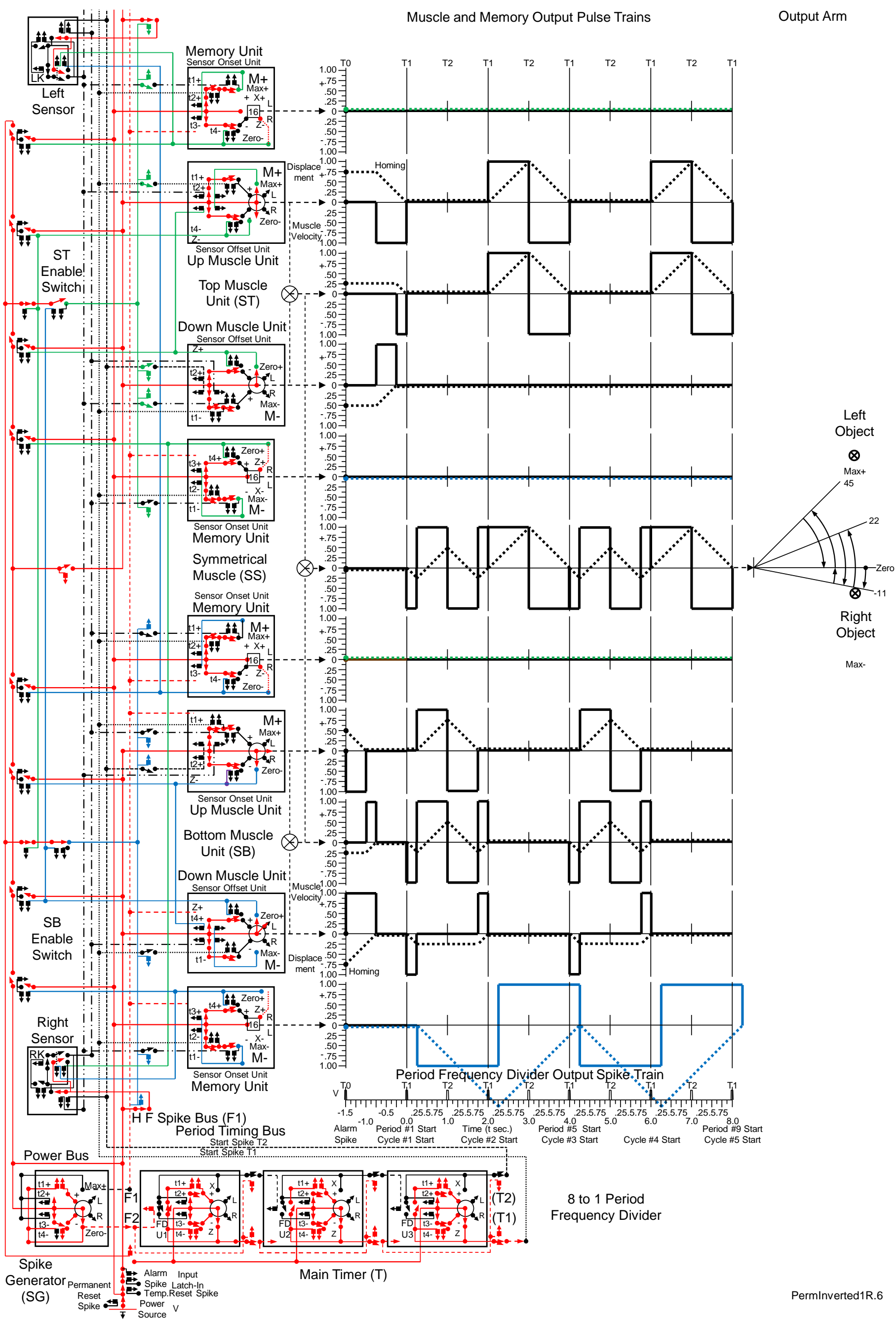

Figure 43 When there is an object on the right side of the Output Arm, the Down Onset Memory Unit records the time of contact, and reproduces it in the next enabled cycle.

The Memory Unit sends a spike to the Right Sensor that causes it to produce a spike as if it had contacted an object.

\subsubsection{When an object is present on the left side of the Output Arm}


When an object is present on the Left-hand side of the Output Arm, a Memory Register at the top of the Top Muscle Unit $(\mathrm{M}+)$ in the Top Muscle Unit (ST) is energized, as shown in Figure 44, below.

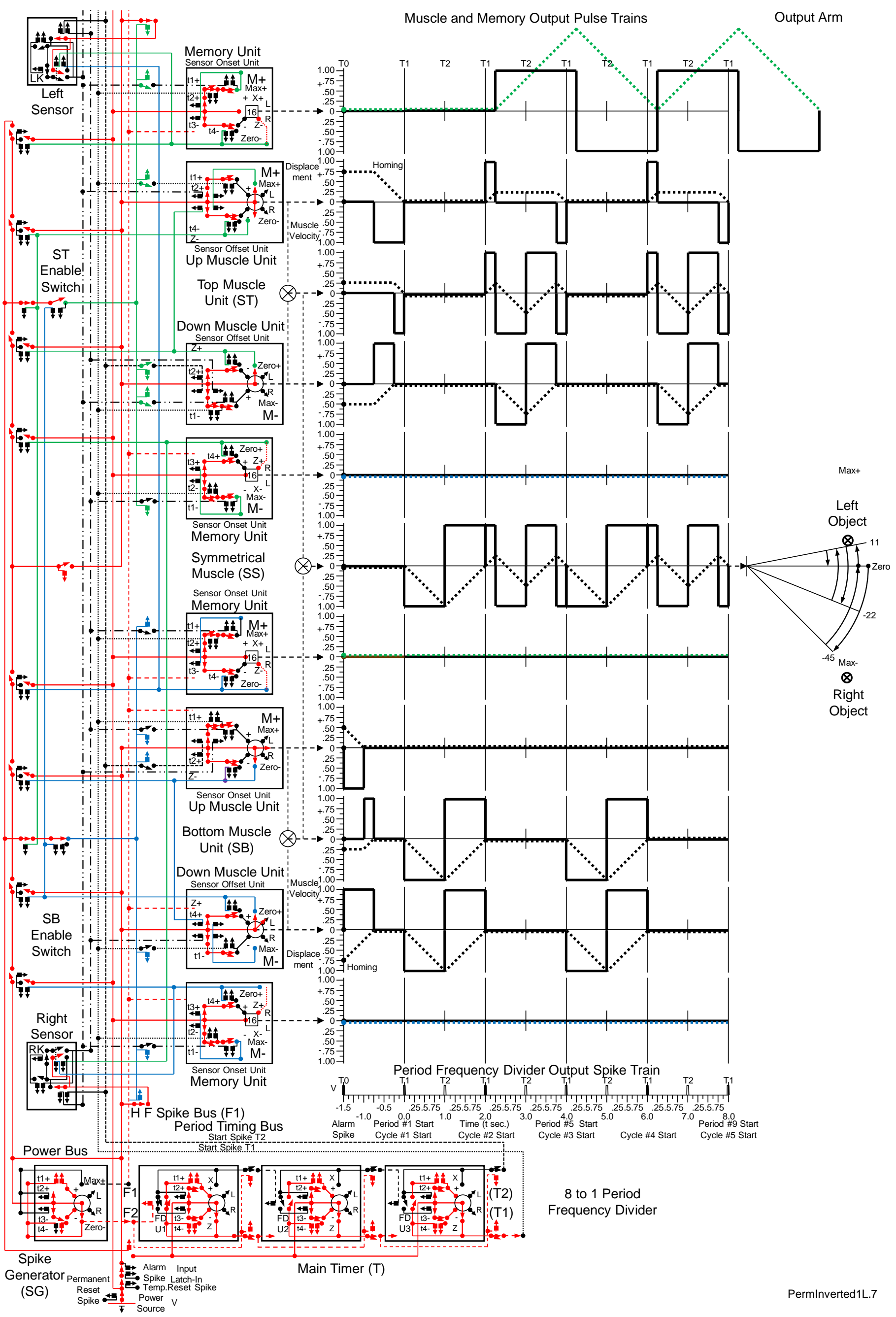

Figure 44 When the object is on the left side of the Output Arm, the Up Onset Muscle Unit records the time of contact, and reproduces it in the next enabled cycle.

The top Memory Unit is connected to the Left Sensor. 
Pulse width modulation

4.7.5 When the Symmetrical Muscle Group contacts objects on both sides of its Output Arm

When objects are located on both side of the Output Arm, the two middle memory registers are energized also, as shown in Figure 45, below.

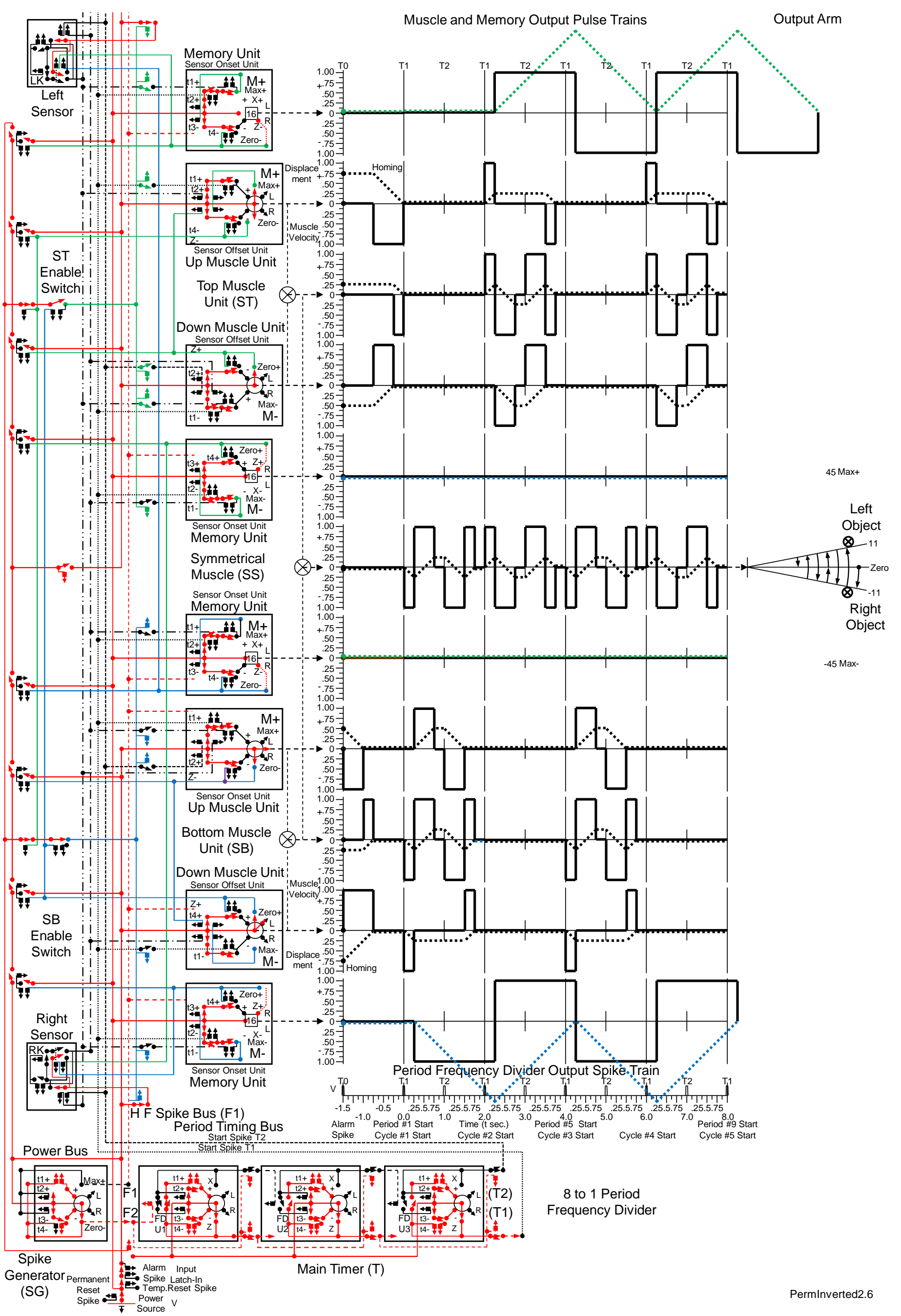

Figure 45 When objects are contacted twice on both sides of the Output Arm, all four Memory Units are activated.

After the first two Cycles, the Memory Units cause the muscle to move in the original pattern even if the objects 
were not present.

\subsubsection{Two objects placed anywhere on either side of the Output Arm}

The object may not be distributed equally on both side of the Output Arm, as shown in Figure 46, below.

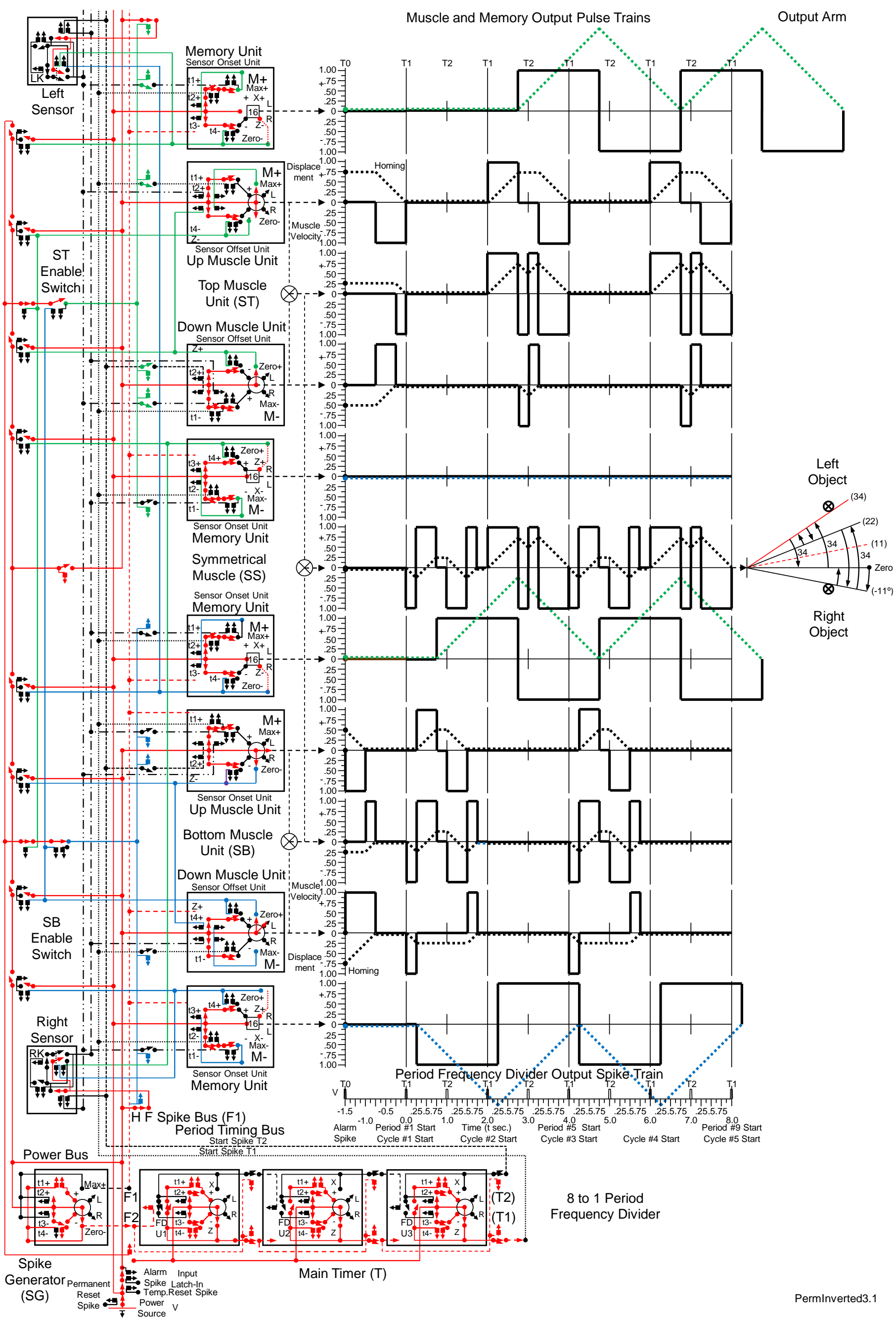

Figure 46 When objects are present on both sides of the Muscle Output Arm, but one object is far enough away from the other, contact is made twice on just one side of the Output Arm. So, one of the Memory Units is not activated. 


\section{Pulse width modulation}

The new off center line of motion objects allows an animal to navigate a burrow without contacting its walls. Even though the midpoint of the motion is shifted off of Zero, all of the muscles have reset to Zero at the end of each enabled Cycle.

\subsection{Permanent memory using a Bi-Directional Frequency Divider used as a Variable Memory Co-Cell}

The frequency dividers used as fixed memories are akin to putting part of the Main Timer into the muscle circuit. Thus, their time value cannot be changed. However, the frequency dividers can be made bi-directional, and can be used as variable timers like the muscles, which record time extents but do not produce motion extents.

\subsubsection{The Bi-Directional Frequency Divider}

The Frequency Divider shown in Figure 23 can be modified so that it can count down as well as counting up, as shown in Figure 47, below.

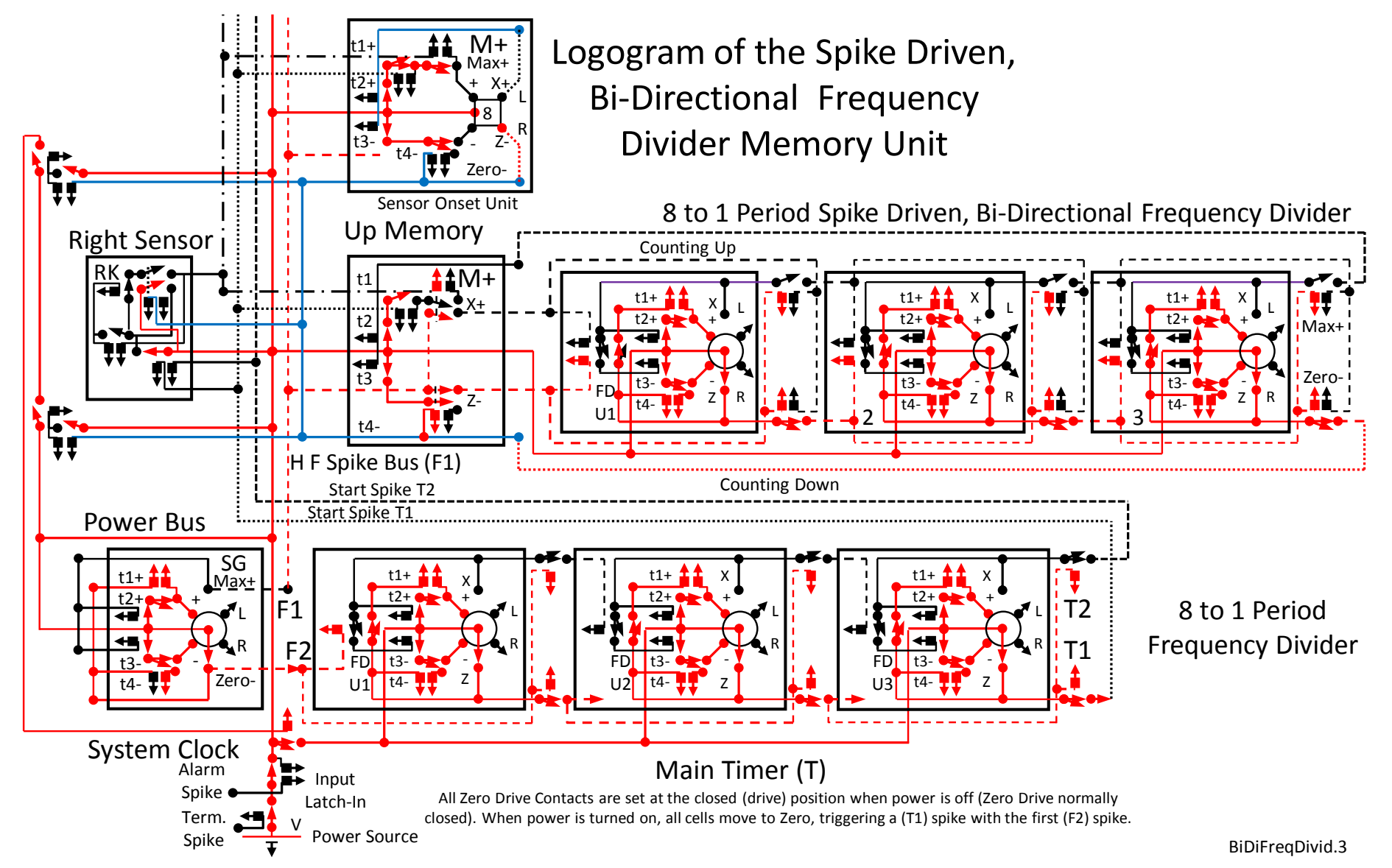

Figure 47 The Bi-Directional Frequency Divider has two connections to the (F1) phase of the High Frequency Spike Train

(F1). One connection (+) causes it to count up, and the other connection (-) causes it to count down.

The Logogram of the Bi-Directional Frequency Divider is the same as the Logogram for the (M+) Muscle shown in Figure 7, except it has a (T1) Enable, and it drives the Bi-Directional Frequency Divider instead of a muscle circuit. Thus, it can store a spike count as duration without any motion extent.

\subsubsection{A muscle circuit using a Bi-Directional Memory Co-Cell}

The Bi-Directional Memory Co-cell shown above in Figure 47 can replace the fixed memory co-cells shown previously, as shown in Figure 48, below. 


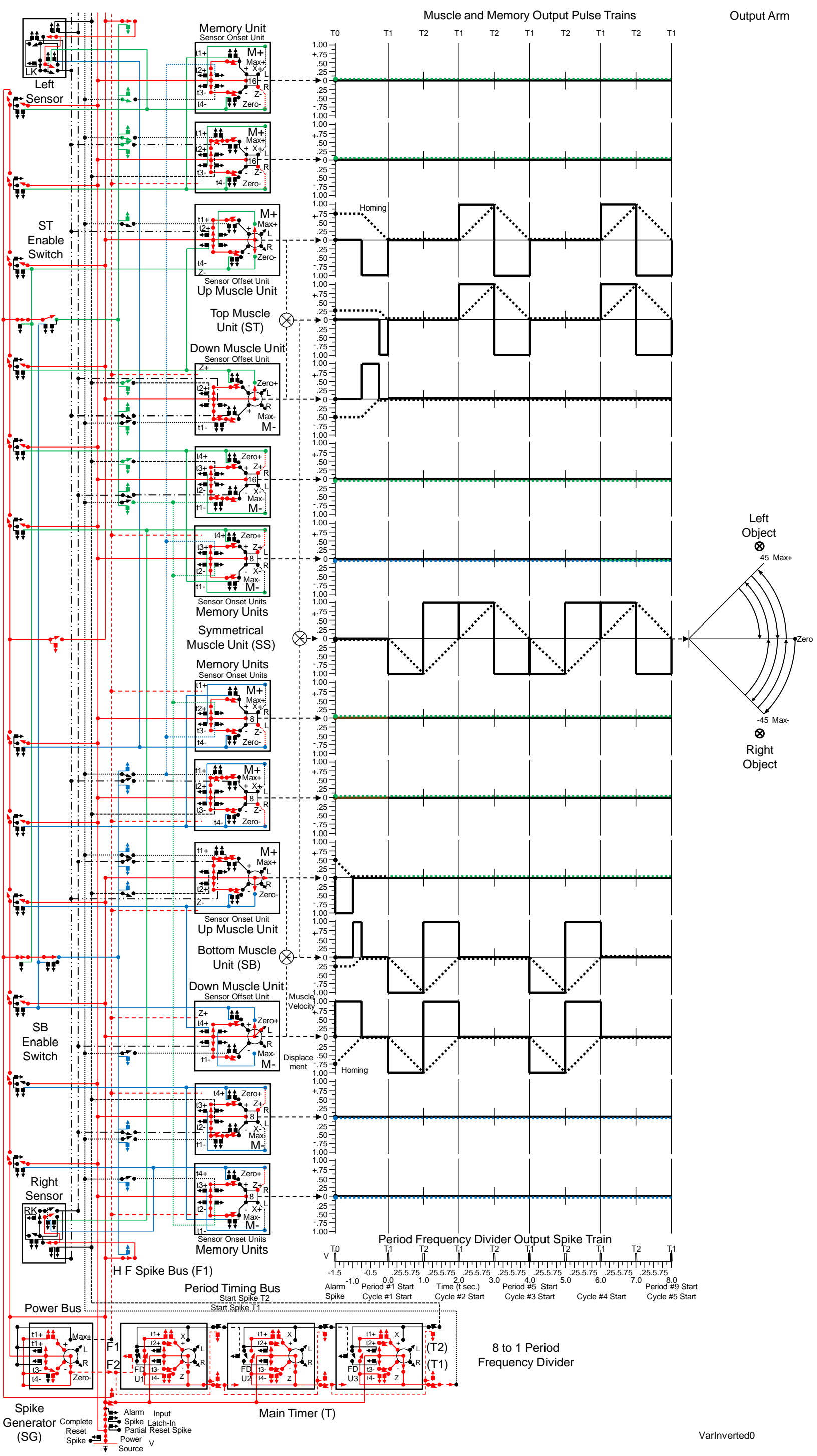

Figure 48 In this example, two Co-Cells are used with each Primary Cell to record the time of contact with an object.

Since no objects are in the path of the Output Arm it moves through its full range of motion.

\subsubsection{When an object is on the right-hand side of the Output Arm}


If an object is present on the right-hand side of the Output Arm, the motions of the Output Arm will be changed, and the location of the object will be remembered by the Bi-Directional Frequency Dividers, as shown below in Figure 49.

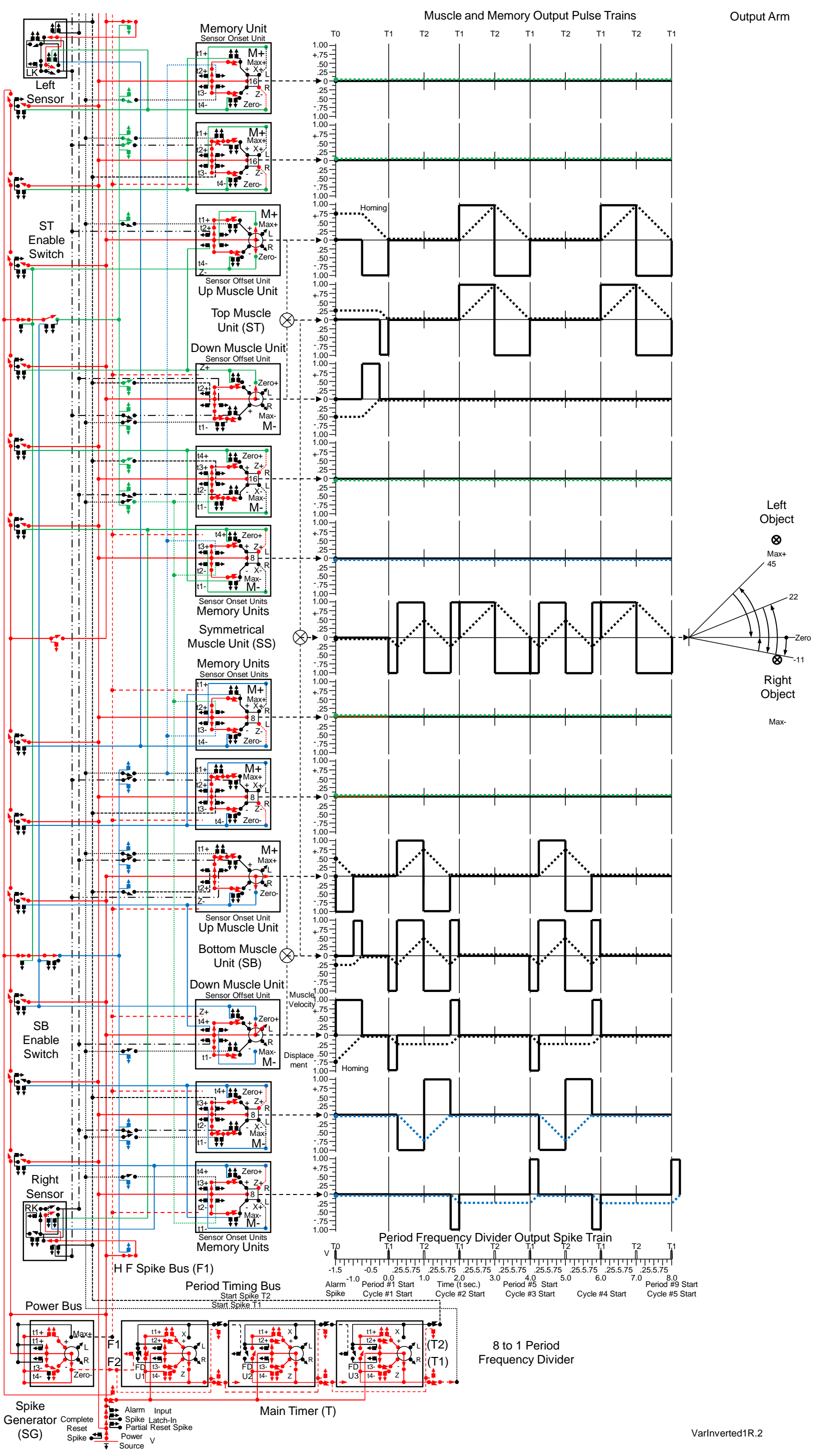

Figure 49 A (M-) Bi-Directional Memory Co-Cell belonging to the lowest Down Muscle (-) is started by the contact with an 
Pulse width modulation

object, stopped and reset at (T2), which starts the other (M-) Memory Co-Cell, which is stopped at the second (T1). It is started at the third (T1), and sends a spike to the Right Sensor when it arrives a Zero, thus reproducing the original timing of the object.

The information about the location of the object is embedded in the carrier frequency (Period Pulse Train), and is decoded by Muscle Output Arm. So the circuit acts like it is being contacted by the original object even if the object were not present.

4.8.4 With the object on the left-hand side of the Output Arm

The object may be located on the left-hand side of the Output Arm, as shown in Figure 50, below. 


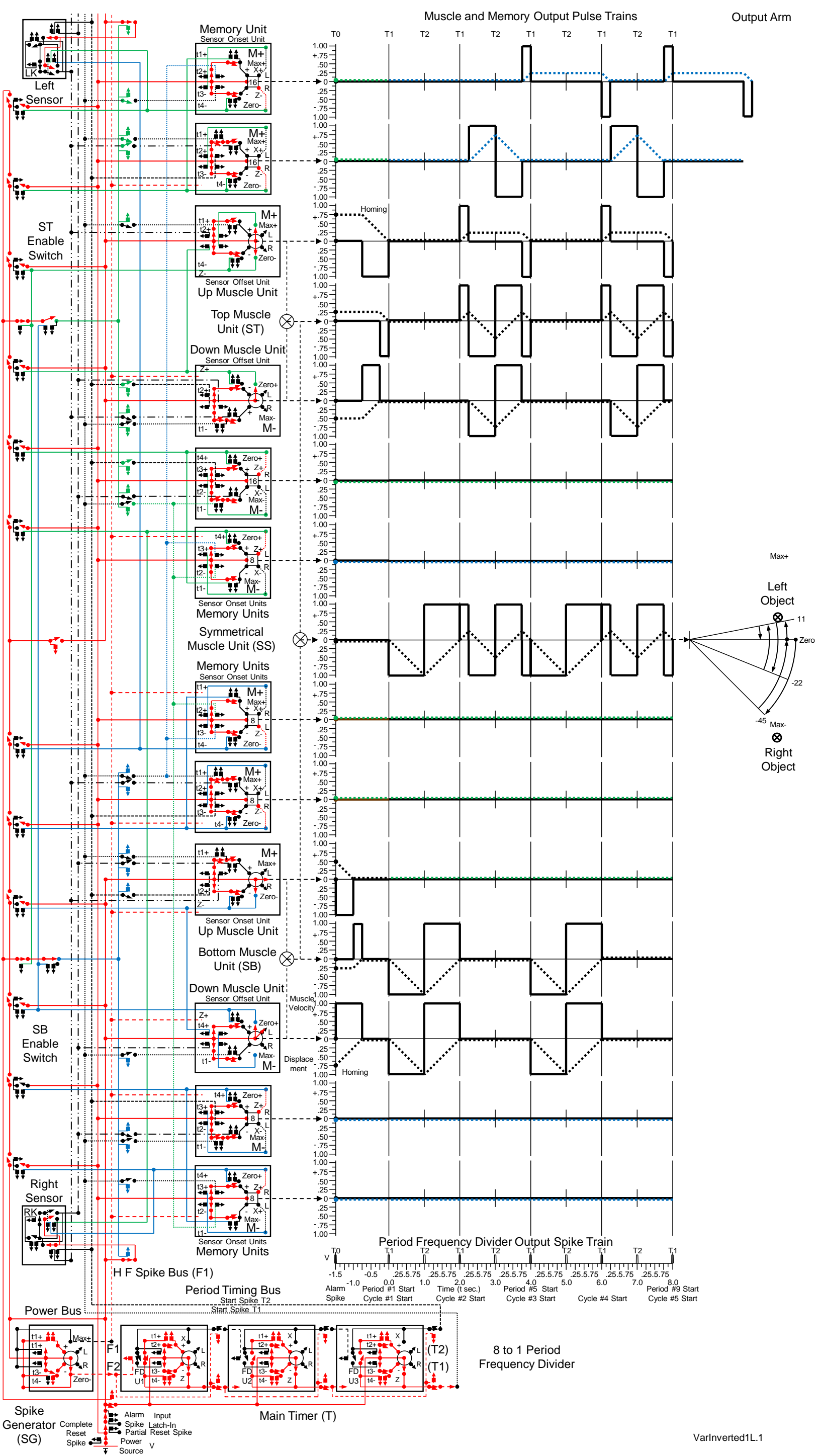

Figure 50 When the object is on the left-hand side of the Output Arm, changes occur in the top Muscle Unit (ST).

The top two Bi-Directional Memory Co-Cells respond to the object as if they were muscles, but they do not produce motion. 
Pulse width modulation

\subsubsection{With objects on both side of the Output Arm}

Objects can be on both side of the Output Arm, as shown in Figure 51, below.

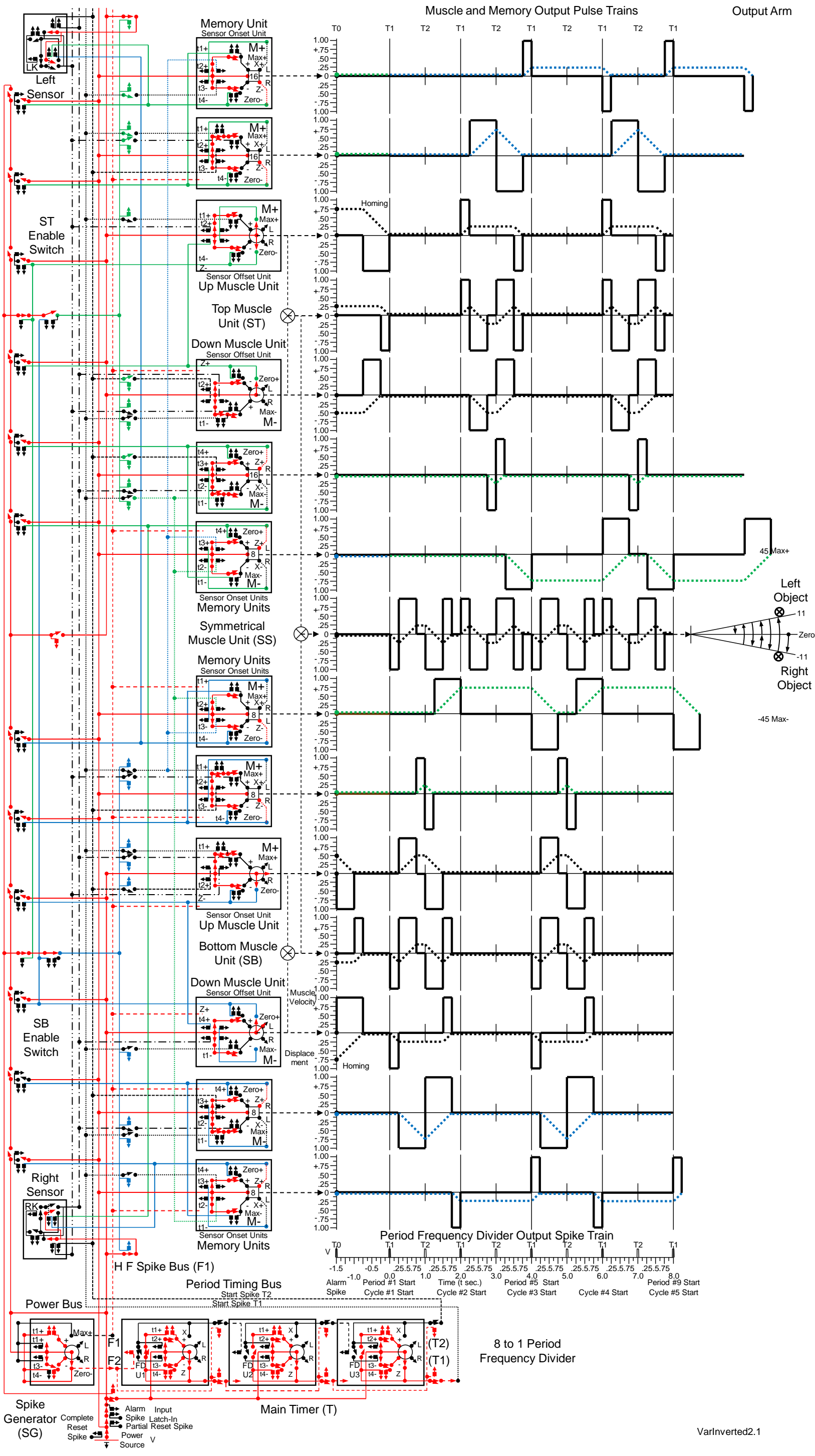

Figure 51 With objects on both side of the Output Arm, all four muscles units and the eight Bi-Directional Co-Cells provide 
Pulse width modulation

the timing needed to keep the Output Arm moving between the positions of the two objects even if the objects were removed after the first contact.

The Bi-Directional Memory Cells contain the information as the location of the two objects, and can be read out by the action of Period Pulse Train (carrier).

\subsubsection{When objects are scattered on both side of the Output Arm}

The objects may not be evenly distributed around Zero, as shown in Figure 52, below. 


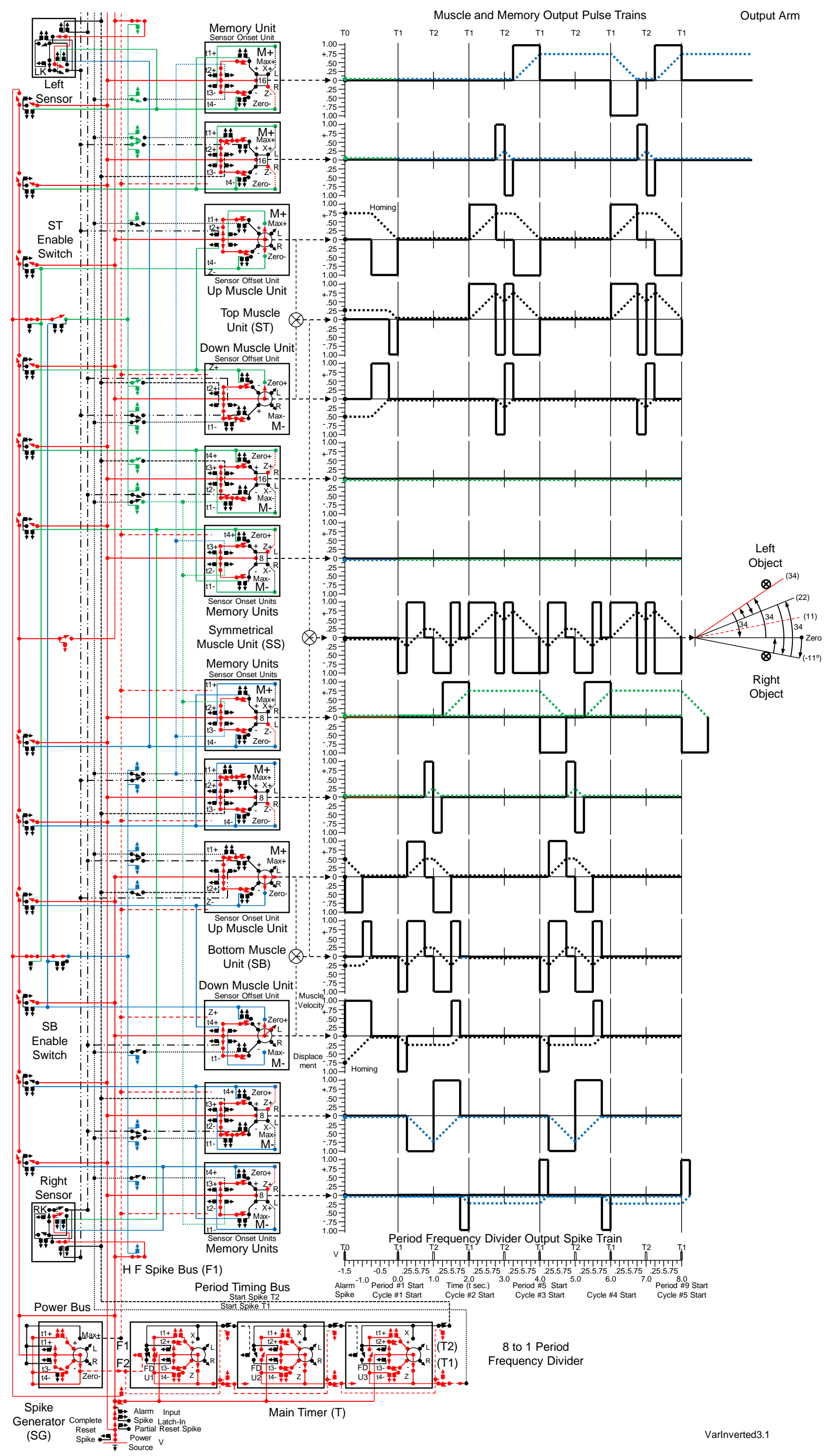

Figure 52 Even though the centerline of motion is not zero, all muscles and memories home to Zero at the end of each active Cycle.

These Bi-directional Co-Cells will be used to providing the timing needed to produce the constant/deceleration of 
Pulse width modulation

the eye saccades in the next section.

\subsection{Eye motion with constant acceleration and deceleration}

All of the motions shown so far consist of right-hand or left-hand motions at a constant velocity. These require an infinite acceleration and deceleration from zero unless there is some mechanical flexibility in their structure. This may work with small flexible animals with little mass. But larger animals (and mechanical robots) work better using changes in velocity having a constant acceleration and deceleration. This constant acceleration and deceleration is produced by a constant force of some magnitude that is determined by the force produced by the muscles and the load, mass, strength, and stiffness of the structure, all of which can be constant.

An example of this constant acceleration/deceleration motion in an animal (human) is given in a paper (Bayhill, 1975 ) in which the motions of the eyeball (saccades) were measured to have constant acceleration and deceleration in what was called the Main Sequence $\left(\mathrm{t}^{2}\right)$ motion by means of a "pulse width modulation controller" using electrical analogue circuitry. The following circuit diagrams are shown of a design for a PWM controller using the more biological components shown so far in this paper.

\subsubsection{Main Timer using Frequency Divider and Step Register}

In order to produce the acceleration/deceleration motion, The Standard Period Pulse Train must be divided into multiple periods that start multiple muscles in sequence. So the Main Timer (T) circuit requires a Four (4) Step Register driven by a 4 to 1 Frequency Divider to start, sequentially, the motion of the 4 eyeball muscles used in this example. This Four Step Register is almost identical to the frequency divider shown previously in Figure 23, except that each unit cannot be reset individually, as shown in Figure 53, below.

\section{Spike Driven Step Register}

Right Stepping (Up Counting)

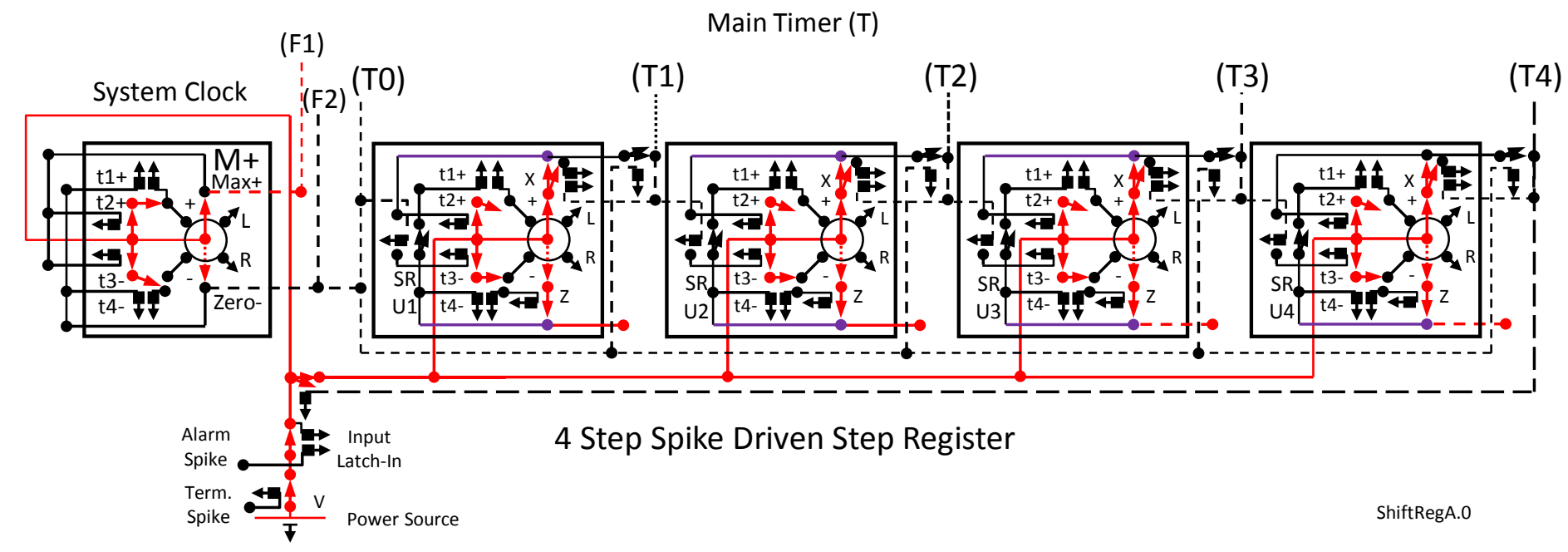

Figure 53 Each Step Register Unit (SRU) produces a spike output, sequentially, like a muscle. But it does not produce a change in movement.

When the Step Register is driven by a 4 to 1 Frequency Divider, it steps (indexes) to the right after every four spikes from the System Clock, and resets itself after the last (T4) output, as shown below. The Step Register Units (SRU's) are just a slight modification as the Frequency Divider Units (FDU's) shown in Figure 21.

\subsubsection{The Pulse Width Modulation Controller needed to produce constant acceleration and deceleration}

The Main Timer shown previously needs to be modified slightly, as shown in the circuit in Figure 54, below. Due to 
limitations of the page size, only the Bottom (SB) Muscle Unit will be shown, with the understanding that a symmetrical Top (ST) Muscle is required in a complete muscle.

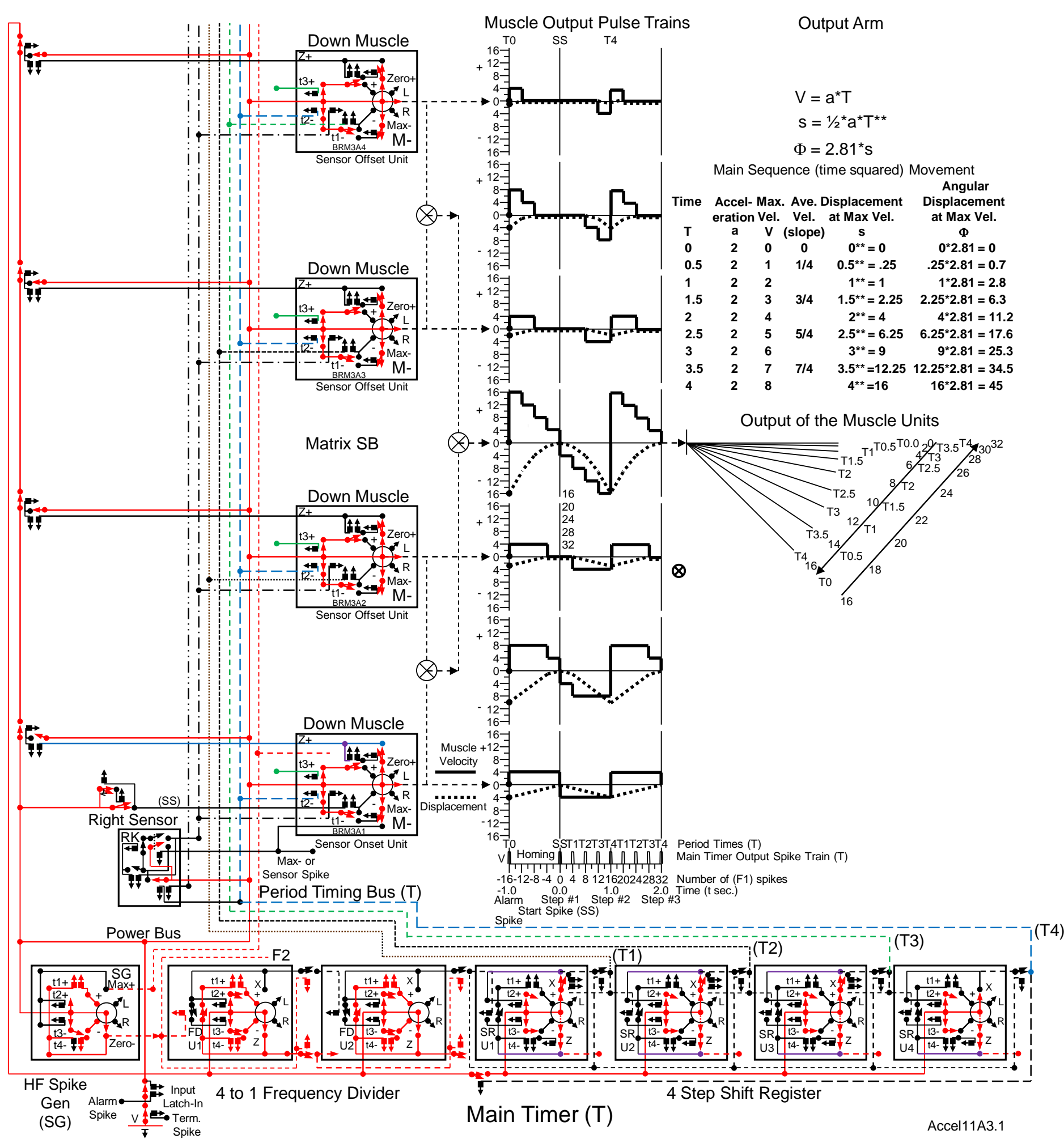

Figure 54 Each muscle unit is started after the bottom muscle unit (BRM3A1) after a specific time delay, and they are all reset at the same time.

This Bottom Muscle Unit (SB) produces an acceleration and deceleration curve. But it reverses direction at its midpoint, unlike the motion of the eye. So an additional circuit, shown in Figure 55, below, is needed to produce the deceleration in the same direction of motion as the acceleration in the eye saccades.

\subsubsection{PWM Controller that can produce the constant acceleration/deceleration of the eye saccades}

The circuit shown above in Figure 54 is modified so that its bottom muscles move in pulses that last twice as long, as shown in Figure 55, below. The time that a pulse reaches zero in the top circuit is used to stop a corresponding pulse in the bottom circuit, as shown in Figure 55, below. 
Pulse width modulation

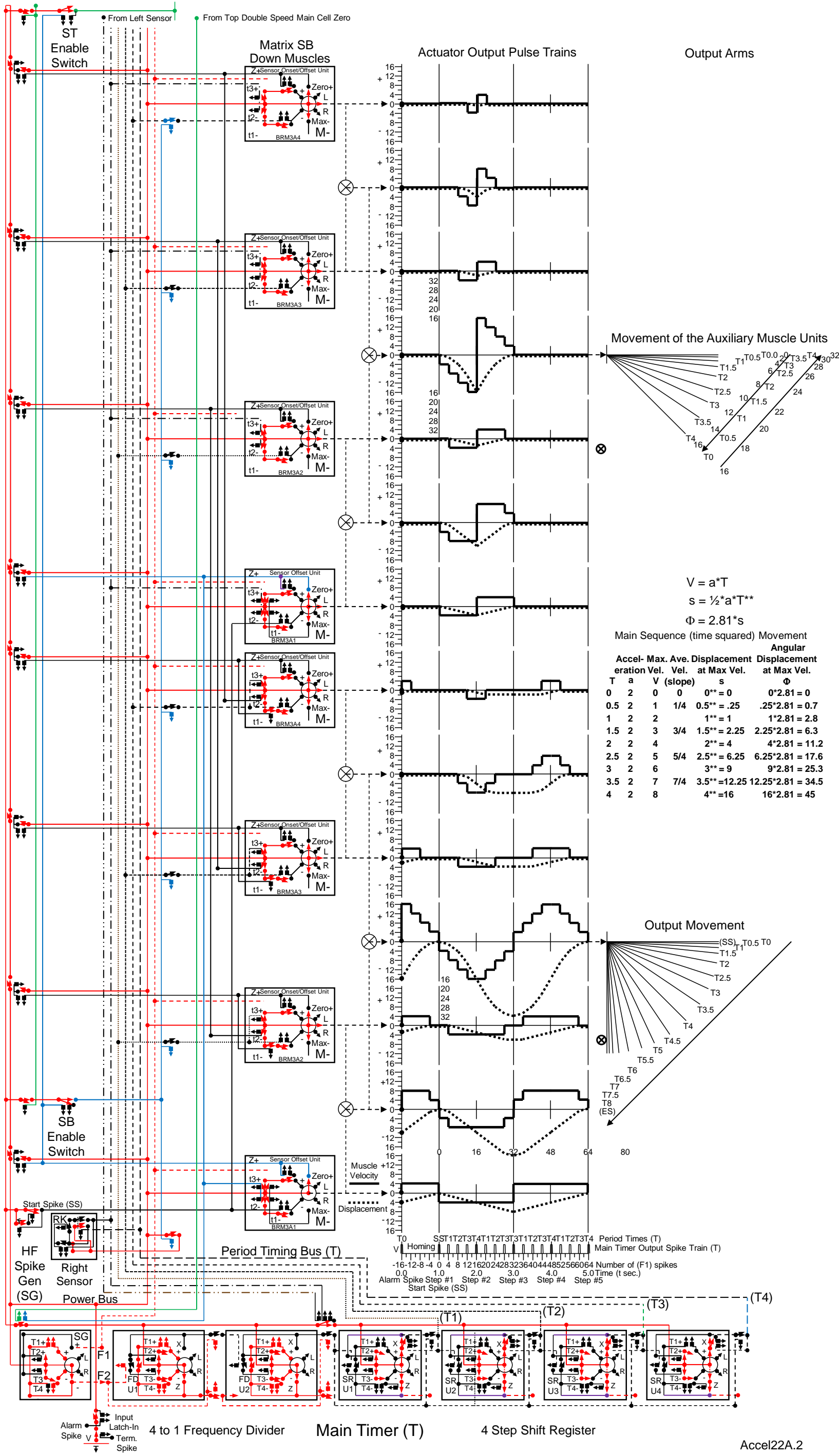

Figure 55 Each of the four bottom muscles are stopped when each of the four top muscles reaches their Zero Position. This timing provides the constant acceleration and deceleration motion in one direction that is produced by the sum of the bottom four muscles in the SB Matrix above.

However, when contact is made with an object, the acceleration must change to deceleration halfway to the point 63 
Pulse width modulation

of contact. This halfway point can be determined in several ways. One way is to use a feeler that moves at twice the speed of the four bottom eye muscles such that the feeler contacts the object when the bottom muscles are half way to the object, as shown below.

4.9.4 PWM Controller needed to produce eye saccades using a double-speed feeler

It turns out that the muscles needed to provide the timing for the main muscles can be used to move a feeler. Since the feeler has to move at twice the speed of the bottom muscles, the feeler muscles can be doubled up (not shown) to produce twice the output. This circuit is shown in Figure 56, below. 
Pulse width modulation

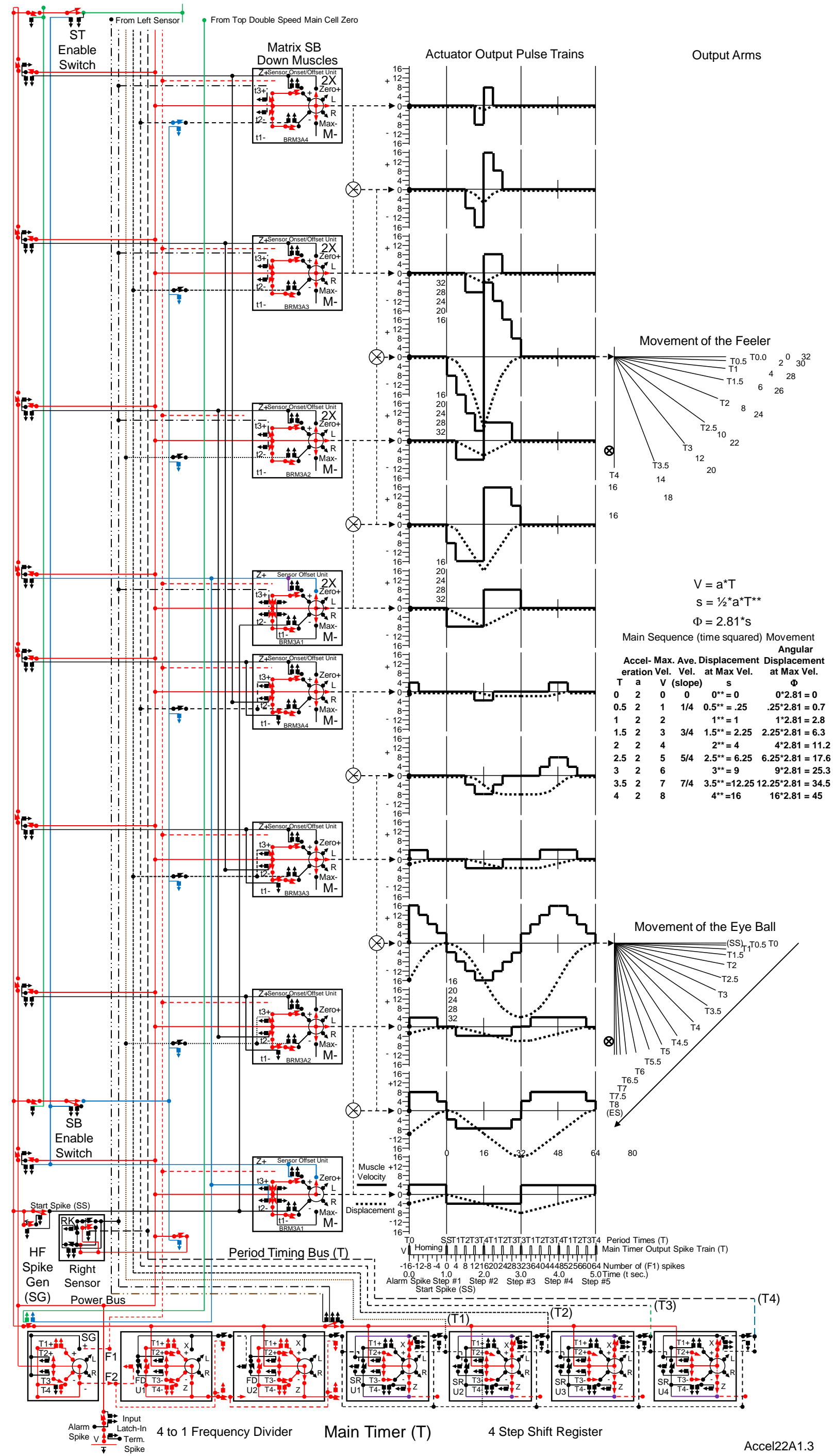

Figure 56 With the feeler muscles traveling at twice the rate $(2 X)$ of the main muscles, the feeler will make contact with the object in half of the time needed by the main muscles.

The feeler not only determines the location of an object, but supplies the timing for the four main muscles of the eyeball to create the constant acceleration and deceleration in one direction and the return to its original position when 


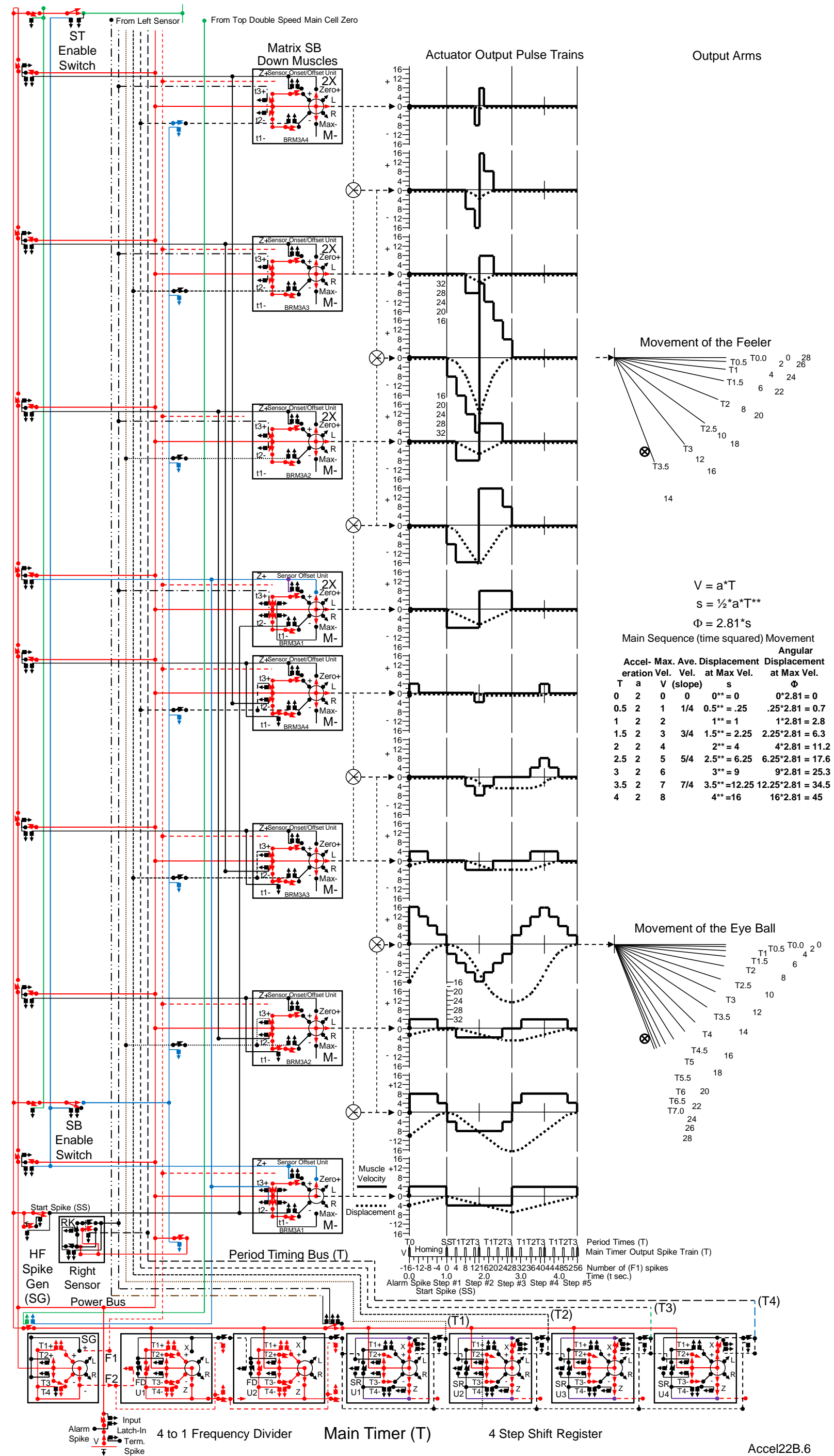

Figure 57 With the feeler making contact at the first T3.5, the main muscles start their deceleration and stop at the second

T3.5. It can complete its motion back to zero and finish at the fourth T3.5 at the beginning of Step \#5.

The return motion to zero needs only the reset motion of the muscles and the timing from the Main Timer to 
Pulse width modulation

produce the needed motion. The return motion re-centers the eyeball, and if the animal uses the timing information of the return motion, it can adjust itself or its head to face directly at the object, as shown later in Figure 61.

\subsubsection{Vision}

Using a feeler to locate an object has some obvious disadvantages. If the object is a predator, touching it with a feeler puts the animal in jeopardy. A far better solution is to sense the object remotely using a vision system. The basic element of the vision system is a half-axis slice of the complete retina shown in Figure 62. The half axis is just one of the two possible positive or negative directions of a complete slice of a complete retina. A slice of a retina can be made using photo sensors having a linearly increasing size with increased distance from the center (zero) point. Each photo sensor is connected to a Step Register Unit (SRU) that is also further from the center (zero) point, and is stepped to zero with each spike from the High Frequency Spike Train (F2) after a SRU it is energized by a photo sensor. Thus, a point of light that falls on the retina at greater distances from the zero (center) point takes the time $\left(\mathrm{t}^{2}\right)$ of the constant acceleration/deceleration curve to step to zero and produce an output spike that starts the deceleration at the half way point to the point of light.

A diagram of this slice of Photo Sensors is shown in Figure 58, below. 
Pulse width modulation

Spikes made active in system with 4 spikes per Standard Period (T), with 4 Standard Periods per Step for 16 Spikes per Step

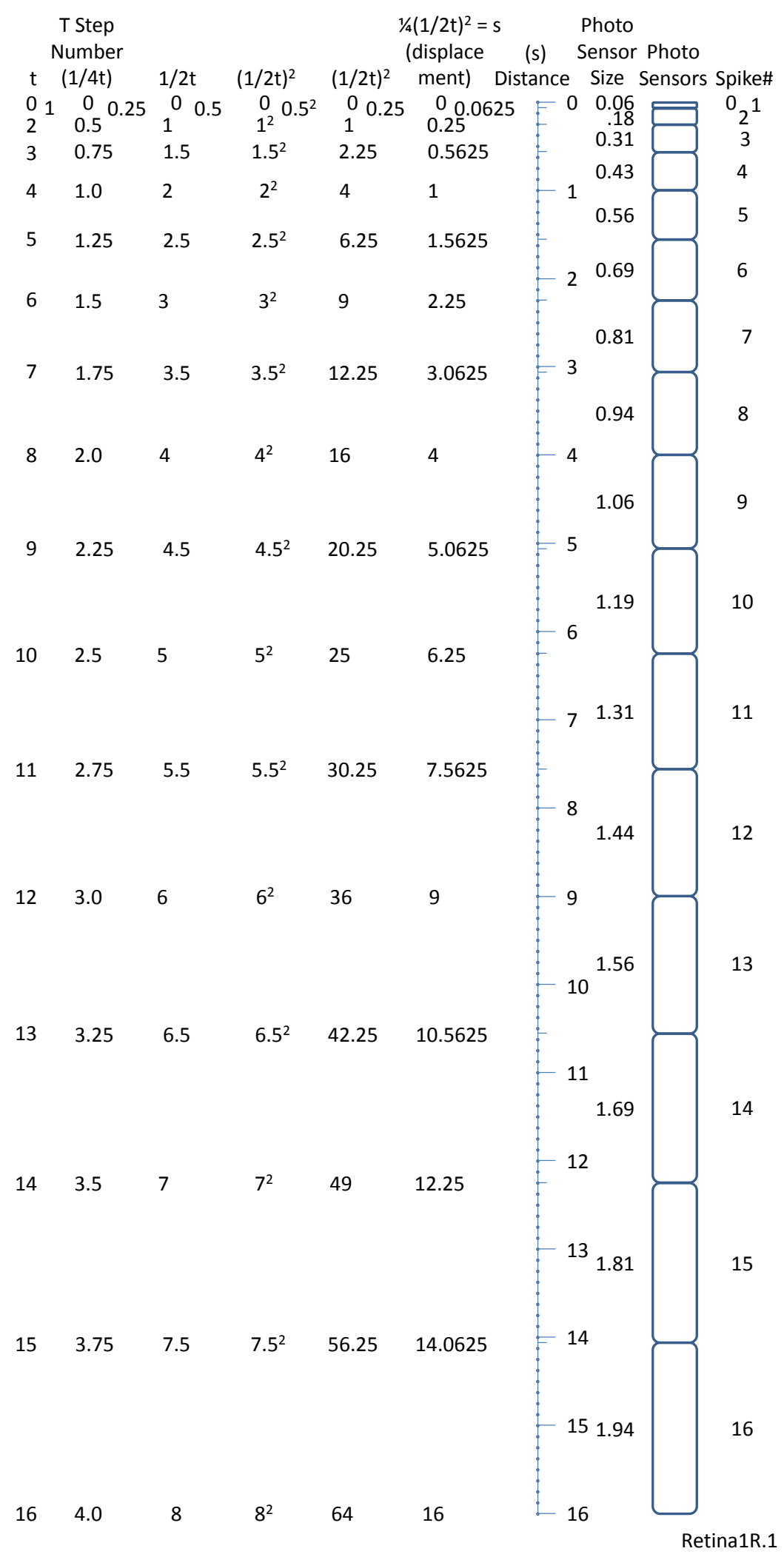

Figure 58 A point of light close to zero would take a disproportionally longer time to cover a given distance to zero through the Step Register than a point of light falling on the retina out near its outer edge.

So the photo sensors are connected to a Step Register, forming a Vision Sensor (RKV) in place of a contact sensor, as shown below in Figure 59. 
Pulse width modulation

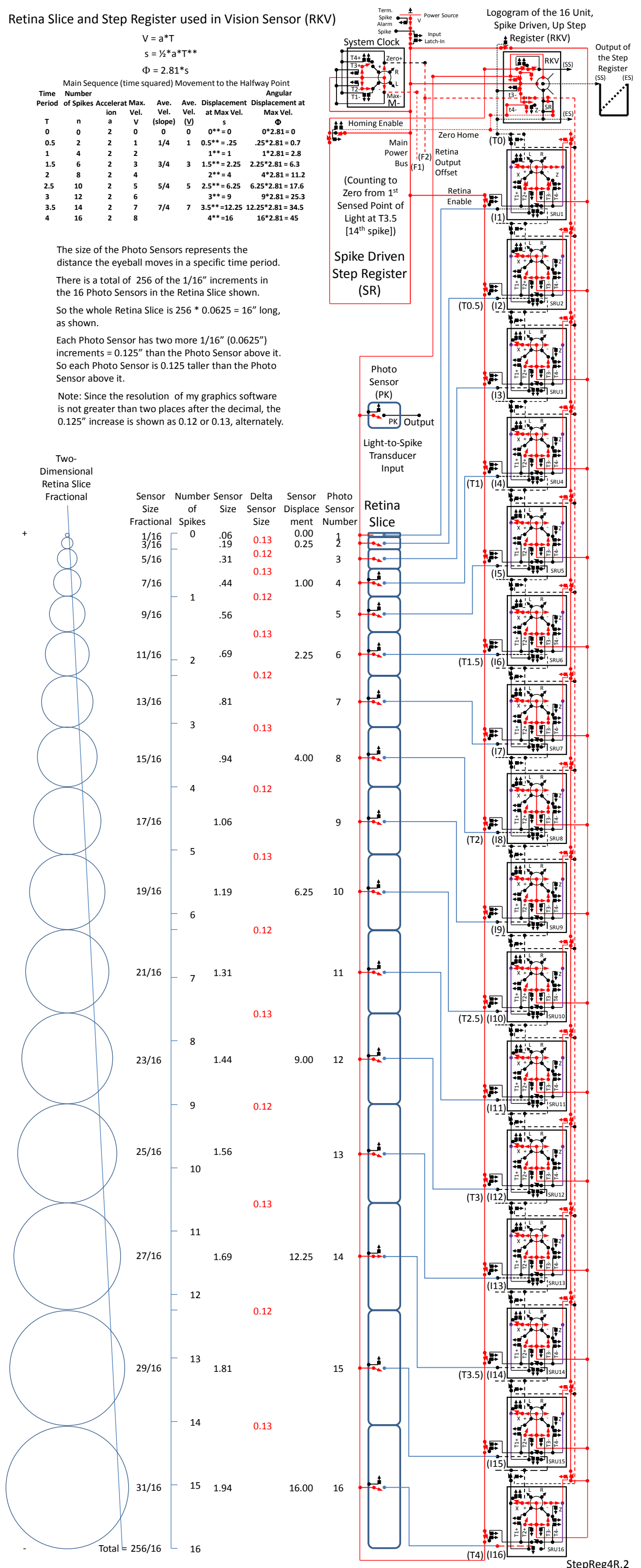


Pulse width modulation

Figure 59 The Retina Slice and Step Register are combined into a Vision Sensor (RKV) that decodes the position of a point of light into a spike time that can be used to transition the acceleration to the deceleration of the eye ball.

A Step Register is connected to the Retina Slice, and is driven by the System Clock such that a point of light falling on the $16^{\text {th }}$ Photo Sensor will require 16 input spikes. If the point of light lands on the Photo Sensor that is $1 / 4$ of the way out it would take 8 spikes to get to zero, following the Main Sequence $\left(\mathrm{t}^{2}\right)$ Equation.

Since there is no need for the feeler muscles, these muscles can be replaced by the Bi-Direction Frequency Divider Memory Unit shown previously in Figure 47. The Bi-Directional Frequency Divider Memory can store and read out time information without muscle movement.

4.9.6 Using the vision sensor to determine the midpoint of the eye motion

So the PWM Controller using the Vision Sensor (RKV) uses the timing information from Retina and Step Register and the Bi-Directional Frequency Diver Memory to produce the constant acceleration/deceleration measured by Bayhill, as shown on the control system below in Figure 60. 
Pulse width modulation

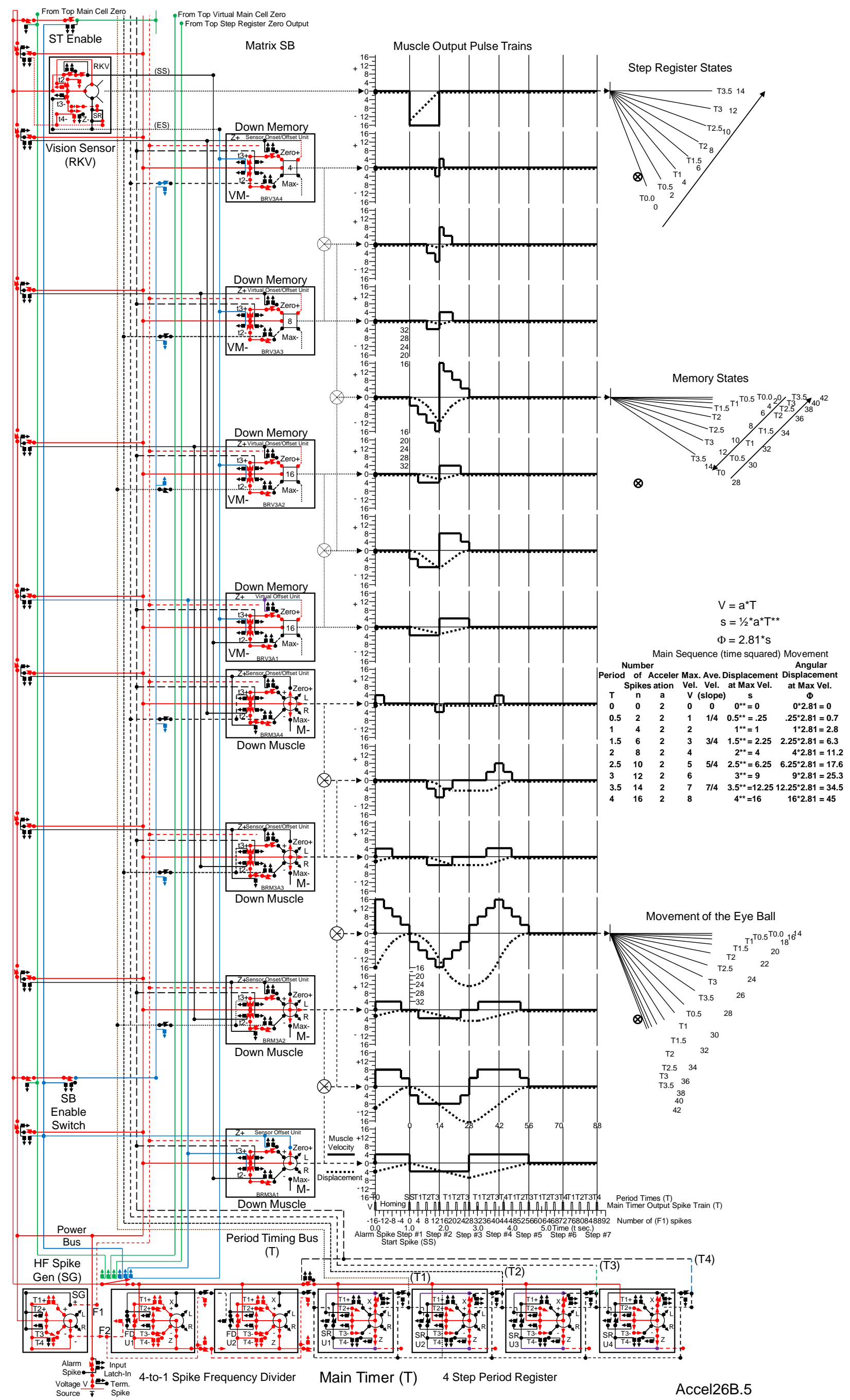

Figure 60 The Retina/Step Register Vision Sensor (RKV) determines the point in time that the acceleration switches to deceleration.

The vision system starts the eye movement the instant that it detects a point of light, and reverses the direction of the acceleration when the eyeball is halfway to the point of light.

4.9.7 The eyeball returns to its center position when the body or head of the animal turn toward the point of light. 
Pulse width modulation

The control system can be expanded to include muscles that accelerate and decelerate the body or head of the animal to turn toward the point of light so that the eyeball can return to its zero (home) position as the head or body turns, as shown below in Figure 61. 
Pulse width modulation

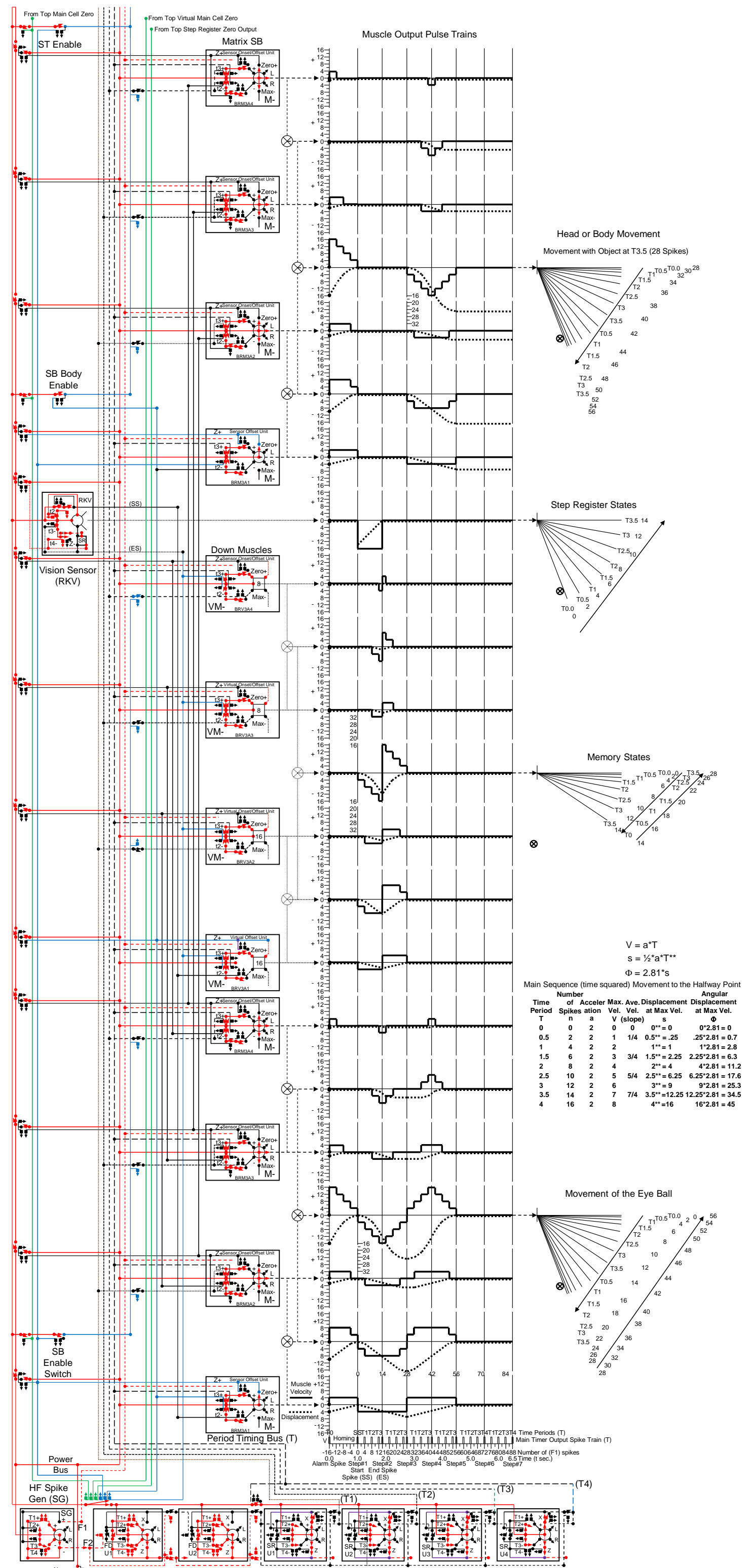

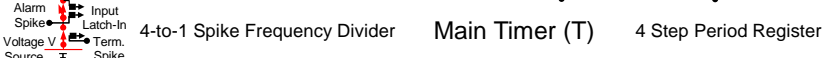


Figure 61 The use of vision allows the eyeball to start turning to a point of light, immediately, and allows the animal to turn and face the point of light while the eyeball returns to its center (Zero) position.

The Retina Slice shown in Figure 59 can be copied and rotated around the zero point to form the complete retina shown below in Figure 62.

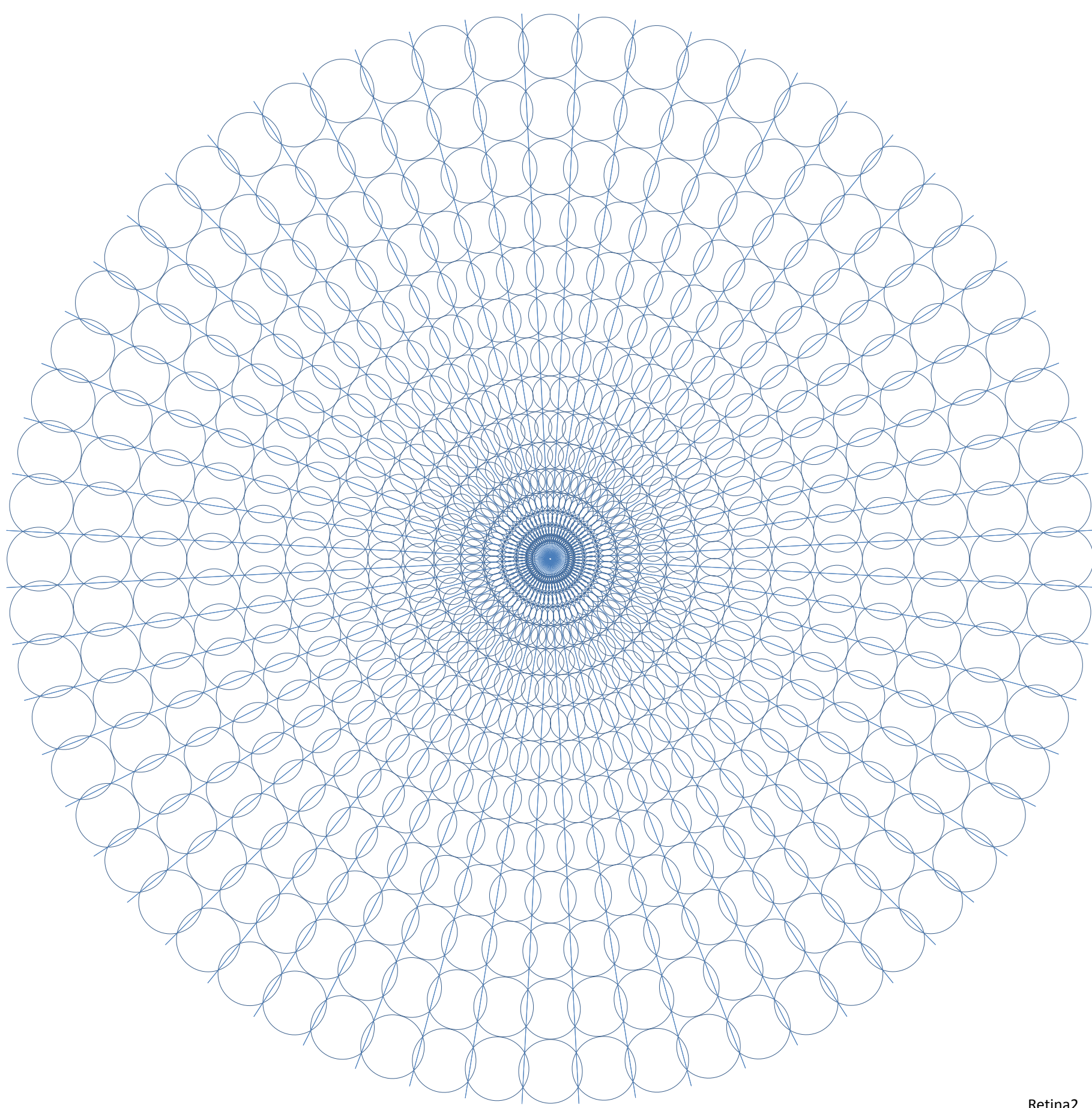

Figure 62 The Complete Retina uses the symmetry inherent in PWM.

These examples of Pulse Width Modulation are just the tip of the iceberg. There are more examples than I can even imagine of the uses of PWM in vision, hearing, speech, animal navigation, echo location, and the purely mental activities involved with consciousness. I will not have time to finish exploring all of them.

\subsection{Permanent memory using an Extended Range, Bi-Directional Frequency Divider Variable Memory Device}

As amazing as it is the way multiple muscles can produce smoothly accelerating and decelerating motions using PWM, an even more amazing feature of PWM is the ability to store and retrieve duration information in an extended range, bi-directional frequency divider (counter) operating off of two memory cell circuits, as shown Figure 63, rather than using separate counters for each memory circuit, as shown in Figure 51, for example. In both cases, the timing of the two memory cell circuits are added together to obtain useful results. However, one extended range counter can perform 
Pulse width modulation

the same addition with almost half of the elements required for the two separate counters. This feature gives a PWM memory system that is equivalent to the memory systems used in the Von Neumann digital computers, and allows the memory system using PWM to perform the needed computations by themselves.

\subsubsection{The extended-range, bi-directional frequency divider variable memory}

Instead of using the two complete memory cells shown in Figure 48 through Figure 52, two memory cell circuits can drive one memory unit, as shown in Figure 63, below.

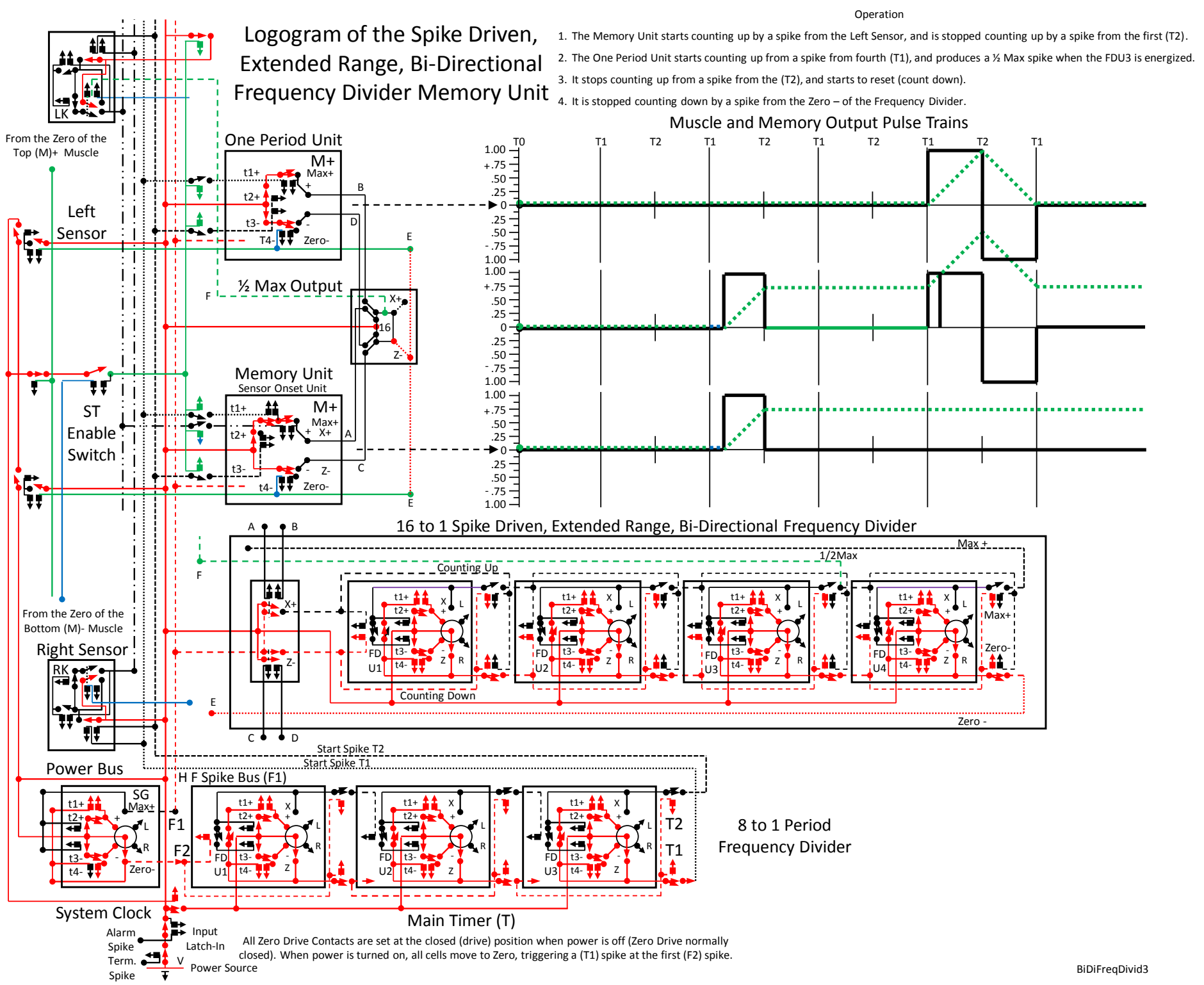

Figure 63 The Extended-Range, Bi-Directional Frequency Divider uses just one more FDU4 that doubles the range of the counter. An output at FDU3 represents the halfway point to the new Max.

A Sensor Onset Unit starts the counter, which is stopped at (T1). The One Period Unit adds a full Standard Period to the counter in the next active cycle. Then, a $1 / 2$ Max Output is produced by the (FDU3) Unit as the count continues to its Max and then resets. The time of the 1/2 Max Output corresponds to the time of the start of the Onset Unit due to a spike from the Left Sensor. This latent spike time remains in the system indefinitely, and can be readout at any time by activating the One Period Unit, which, essentially just adds and subtracts one Pulse Period to the counter.

\subsubsection{The latent memory muscle circuit}

The One Period Unit and the Sensor Onset Unit can replace the Memory Units in the Variable Memory Muscle Circuit shown in Figure 47, as shown in Figure 64, below. 
Pulse width modulation

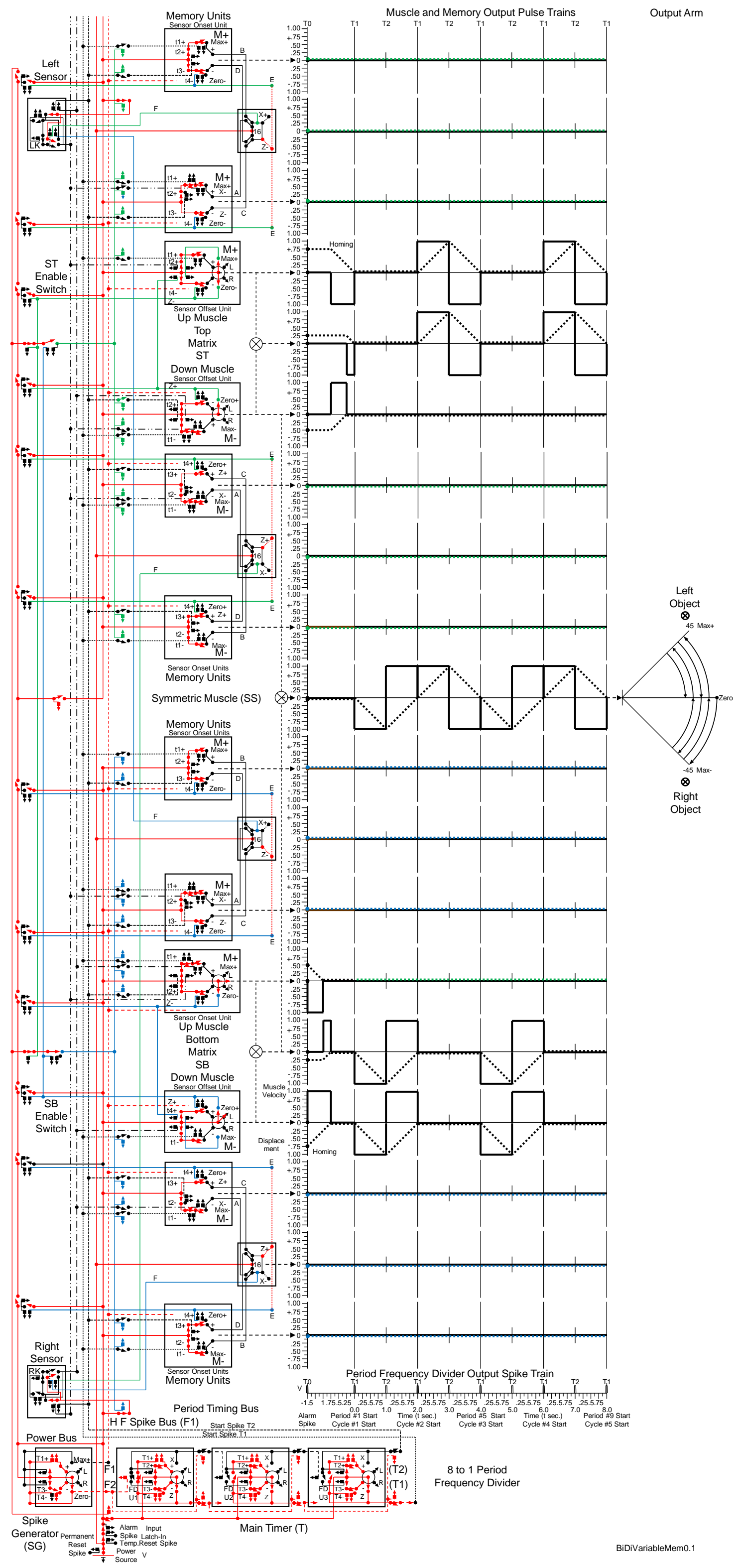


Pulse width modulation

Figure 64 The memory circuits are not activated because there are no objects contacted by the sensors. So the Output Arm moves through its full range of motion.

The memory circuits do not produce motion. So they are not connected to the muscle units by a differential.

4.10.3 When there is an object on the right-hand side of the Output Arm

There may be an object on the right-hand side of the Output Arm, as shown in Figure 65, below. 
Pulse width modulation

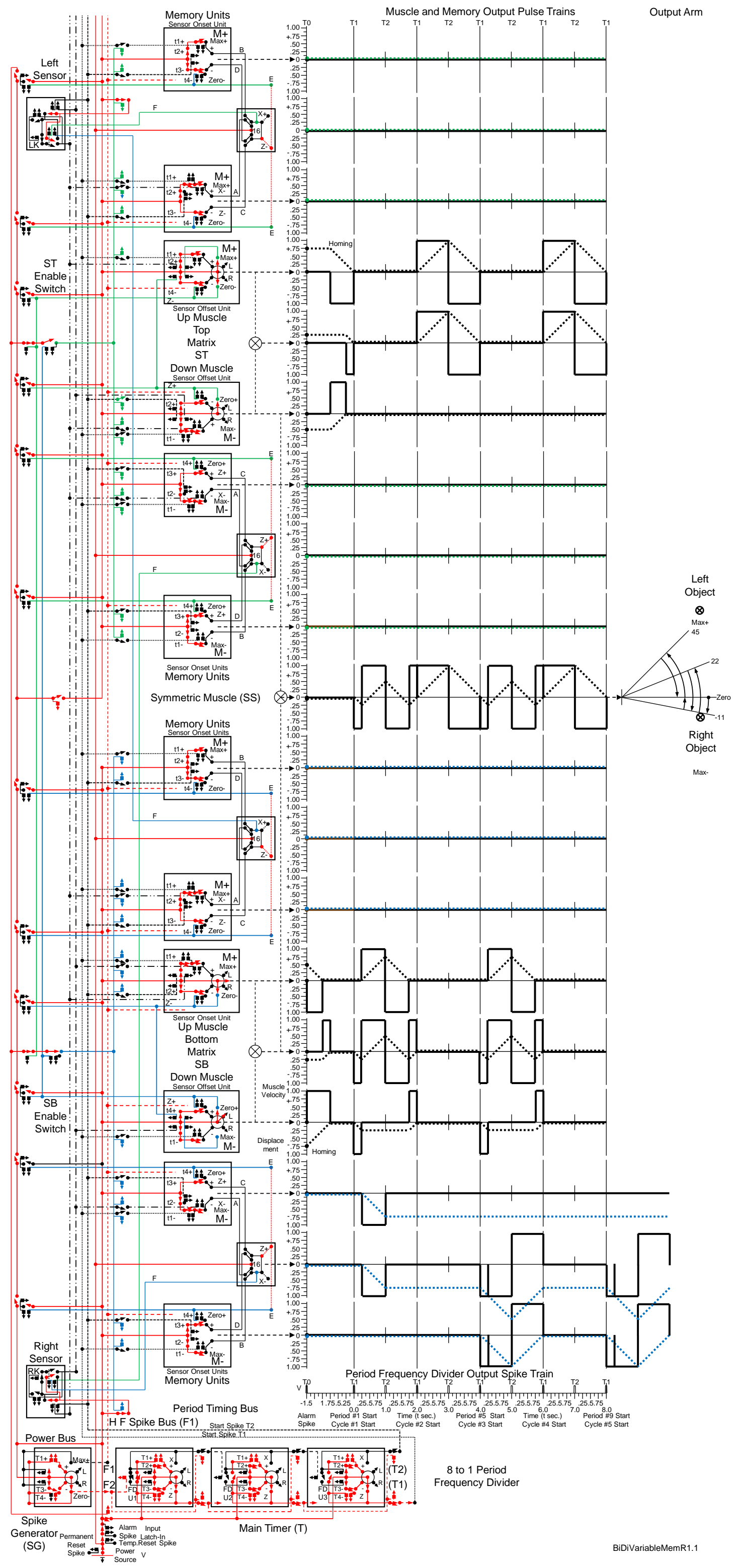


Pulse width modulation

Figure 65 The Onset Unit is started by the Right Sensor, and is stopped by (T2). The Onset Unit cannot be reset. So the counter remains at this count. The One Period Unit was not started by the first (T1) because it has to be enabled by a sensor spike.

The memory units are connected to the sensors, only.

4.10.4 When there is an object on the left-hand side of the Output Arm

There may be an object on the left-hand side of the Control Arm, as shown in Figure 66, below. 
Pulse width modulation

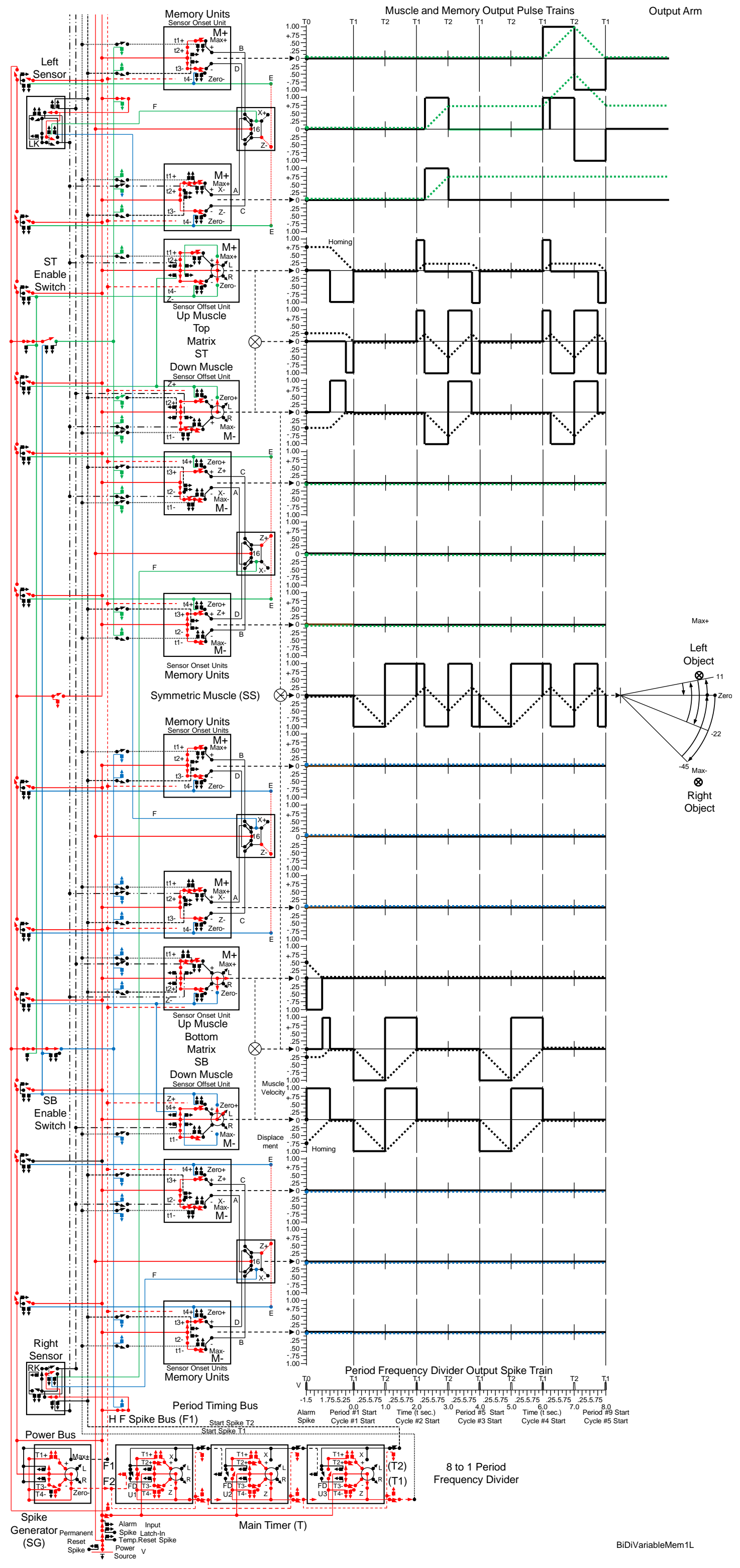


Pulse width modulation

Figure 66 The memory units in the Top Matrix (ST) are energized by the Left Sensor.

The memory units just keep track of time extents within the Standard Periods.

4.10.5 Objects may be place equidistantly on both side of the Output Arm

There may be objects placed equidistantly on both sides of the Output Arm, as shown in Figure 67, below. 
Pulse width modulation

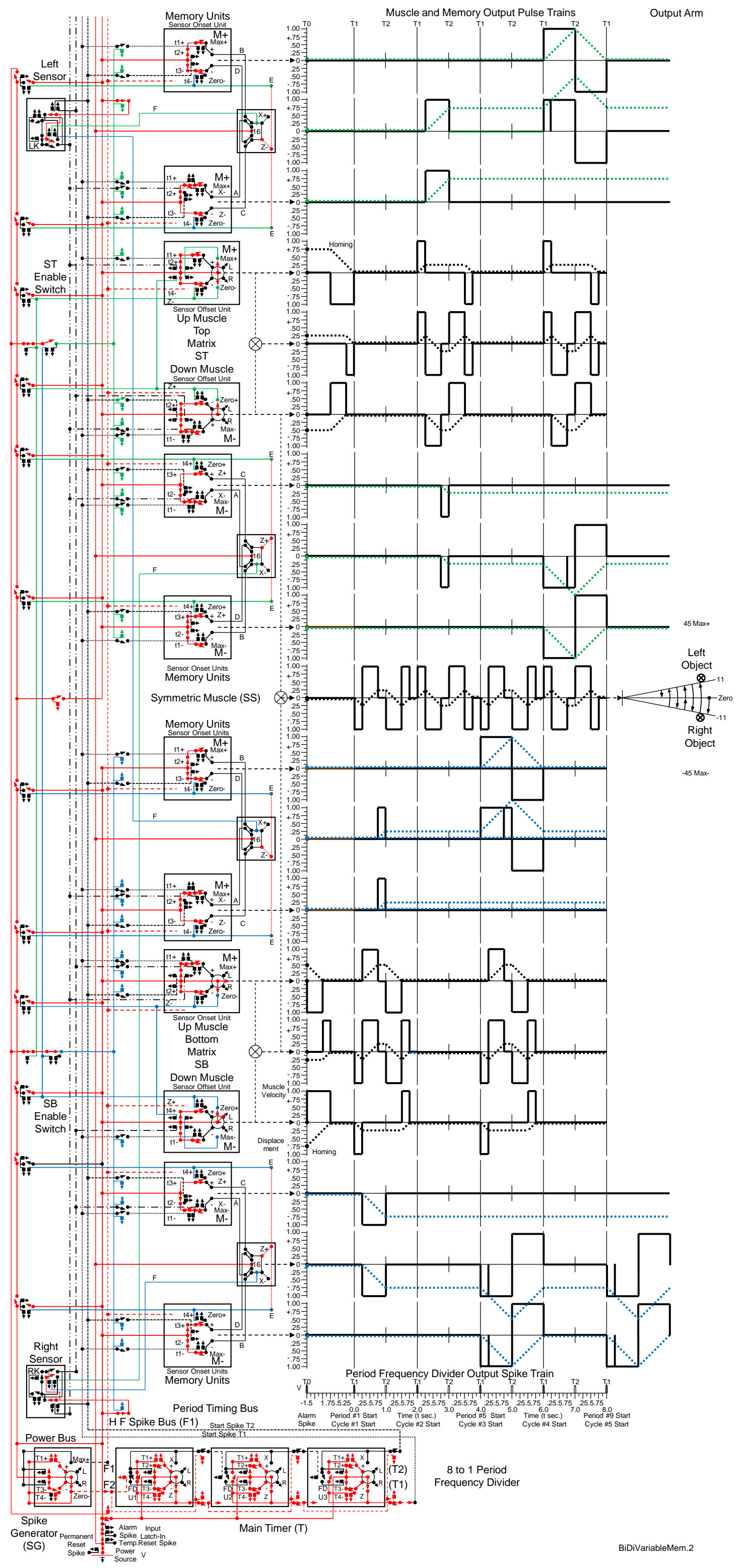


Pulse width modulation

Figure 67 All of the memory units may be activated when contact is made with objects on both side of the Output Arm.

Because of these latent memories, the Output Arm will continue to behave as if the objects were present.

4.10.6 When the objects are at different positions on either side of the Output Arm

These latent memory circuits can deal with unsymmetrically placed objects, as shown in Figure 68, below. 
Pulse width modulation

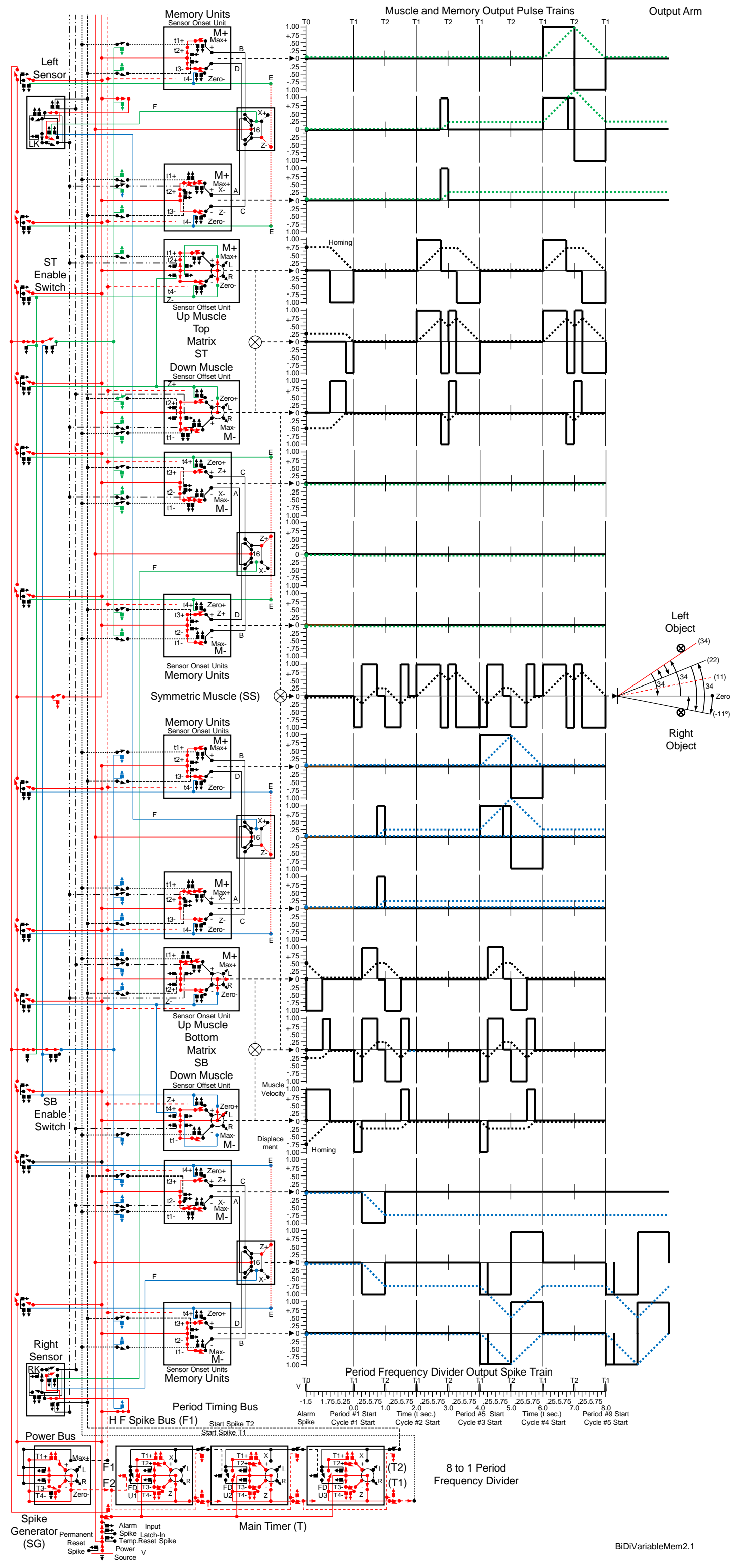


Figure 68 The only cells that are not reset to Zero are the Sensor Onset Cells. In this unsymmetrical distribution of objects, only one Sensor Onset Memory Cell remains at a zero value.

The design of these latent memories provides the foundation of a memory and computation process that closely resembles the memory devices in a digital computer. The difference between the up/down counters shown above and the computer binary memory register is that an up/down count is read out in series, and a computer register can be read out in parallel. But the PWM system uses the duration of these series readout of the counters as the source of information rather than the decoded value of the binary registers in computers, and the PWM system can compute using these durations directly without arithmetic logic. This may be the most compelling argument for more investigations into the use of PWM in the brain.

\section{Conclusion}

This paper shows how Pulse Width Modulation (PWM) can be used to reproduce the behavior found in animal biology. Since PWM is the science behind rhythm, it is the foundation (language) of music, speech, dance, echo location, sonar, telegraphy, and the original Fax telegraphics. So it stands to reason that the brain uses PWM to produce and understand music and speech and deal with these the other movement and messaging processes involving rhythm. Applying the principles of PWM to vision, hearing, speech, animal navigation, echo location, and the purely mental activities involved with consciousness could lead to the same level of success that has been shown in this paper to explain biological processes such as how muscles and memory work, and how eye saccades are produced using PWM. The science of PWM is seldom if ever reported or referenced in the field of biology and neuroscience. The foundation of PWM is the use of spike times within repeating carrier time pulses to encode information. Brain waves may well be the periodic time pulses used by PWM as the time framework carrier to encode the information in the brain. PWM controllers are used to create the bursts of pulses in transcranial stimulation of the brain, which are reported to produce desirable changes in the brain, which also suggests that PWM is the foundation of the brain. So the field of biology and neuroscience will benefit greatly by learning more about PWM, and applying it to the other activities of animals to better understand the language of the brain.

\section{Bibliography}

Bahill, A., Clark, M., \& Stark, L. (1975). The Main Sequence, A Tool for Studying Human Eye Movements.

Mathematical Biosciences 24, 191-204.

Bahill, A., Clark, M., \& Stark, L. (1974). A pulse-width modulated model for visual eye tracking. Berkeley: University of California.

Brown, R. A. (1967). Patent No. 3,325,732. USA.

Brown, R. A. (1994). Machines that learn. New York: Oxford University Press.

Brown, R. A. (2011). A spike processing model of the brain. Nature Precedings.

Brown, R. A. (2016). The design of a proto-animal brain based upon spike timing. arxiv.org.

Buzsaki, G. (2006). Rhythms of the brain. New York: Oxford University Press. 